\title{
Supersymmetric index from black hole entropy
}

\author{
Atish Dabholkar, ${ }^{a, b}$ João Gomes, ${ }^{a}$ Sameer Murthy ${ }^{a}$ and Ashoke Sen ${ }^{a, c}$ \\ ${ }^{a}$ Laboratoire de Physique Théorique et Hautes Energies (LPTHE), \\ Université Pierre et Marie Curie-Paris 6; CNRS UMR 7589

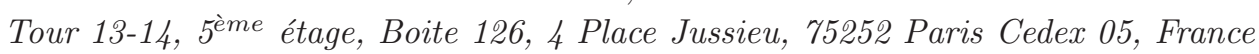 \\ ${ }^{b}$ Department of Theoretical Physics, Tata Institute of Fundamental Research, \\ Homi Bhabha Rd, Mumbai 400 005, India \\ ${ }^{c}$ Harish-Chandra Research Institute, \\ Chhatnag Road, Jhusi, Allahabad 211019, India \\ E-mail: atish@lpthe.jussieu.fr, joaomvg@gmail.com, \\ smurthy@lpthe.jussieu.fr, sen@mri.ernet.in
}

ABSTRACT: For BPS black holes with at least four unbroken supercharges, we describe how the macroscopic entropy can be used to compute an appropriate index, which can be then compared with the same index computed in the microscopic description. We obtain exact results incorporating all higher order quantum corrections in the limit when only one of the charges, representing momentum along an internal direction, approaches infinity keeping all other charges fixed at arbitrary finite values. In this limit, we find that the microscopic index is controlled by certain anomaly coefficients whereas the macroscopic index is controlled by the coefficients of certain Chern-Simons terms in the effective action. The equality between the macroscopic and the microscopic index then follows as a consequence of anomaly inflow. In contrast, the absolute degeneracy does not have any such simple expression in terms of the anomaly coefficients or coefficients of Chern-Simons terms. We apply our analysis to several examples of spinning black holes in five dimensions and non-spinning black holes in four dimensions to compute the index exactly in the limit when only one of the charges becomes large, and find perfect agreement with the result of exact microscopic counting. Our analysis resolves a puzzle involving M5-branes wrapped on a 5-cycle in $K 3 \times T^{3}$.

KEYwords: Black Holes in String Theory, AdS-CFT Correspondence

ArXiv ePrint: 1009.3226 


\section{Contents}

1 Introduction and summary of results 1

2 Computing the index in the macroscopic theory 10

3 Macroscopic results for four and five dimensional black holes 18

3.1 D1-D5-p system in type IIB on $K 3 \times S^{1} \quad 18$

$\begin{array}{lll}3.1 .1 & \text { Type IIB Cardy limit } & 19\end{array}$

3.1.2 Type IIA Cardy limit 23

3.2 Entropy of some four dimensional black holes 26

3.3 Black holes in toroidally compactified type IIB string theory 28

4 Analysis of the exterior contribution $\quad 29$

5 Microscopic results $\quad 33$

5.1 D1-D5-p system in type IIB on $K 3 \times S^{1}$

5.2 D1-D5-p-KK monopole system in type IIB on $K 3 \times T^{2} \quad 38$

5.3 Black holes in toroidally compactified type II string theory 40

6 MSW analysis for M5-branes on $K 3 \times T^{3}$ and $T^{7} \quad 42$

6.1 M5-brane on $K 3 \times T^{3} \quad 42$

6.2 M5-brane on $T^{7} \quad 44$

7 Why do the microscopic and macroscopic results agree? 44

A Chern-Simons contribution from higher derivative terms 49

B Asymptotic expansion $\quad 52$

\section{Introduction and summary of results}

In a class of supersymmetric string theories with sixteen or more unbroken supercharges we now have a near complete understanding of the spectrum of BPS states [1-35]. This makes these theories ideal testing ground for a comparison between the statistical entropy of an ensemble of states and the thermodynamic entropy of the corresponding BPS black hole. In particular, given such an exact knowledge of the microscopic degeneracy, one can aim for a possibly exact comparison with an appropriately defined macroscopic entropy that includes all subleading corrections. On the macroscopic side the subleading classical corrections arising from local higher derivative terms in the effective action can be incorporated using the Wald formula [36] whereas the subleading quantum corrections, both perturbative and 
nonperturbative, can be incorporated using the framework of quantum entropy function [37, 38]. These can then be compared with the subleading corrections on the microscopic side after carrying out a systematic asymptotic expansion of the exact formula.

In carrying out such a comparison one needs to be careful about an important subtlety. On the macroscopic side, the black hole entropy defined from the first law of thermodynamics calculates the logarithm of the absolute degeneracy as required by the Boltzmann relation. On the other hand, on the microscopic side, one normally computes a supersymmetric index which receives contribution only from BPS states and hence is protected from any change under continuous deformations of the moduli of the theory. A priori the index and the degeneracy are not the same, and one could question the rationale behind comparing the degeneracy computed in the macroscopic side with the index computed on the microscopic side.

One can proceed nevertheless following the dictum that whatever can get paired up will generically get paired up, and hence in the interacting theory the index equals the degeneracy. In many examples this strategy has worked very well for the leading entropy. However, there is no guarantee that it will work also for the subleading corrections. Indeed, there are a number of puzzles in the context of four-dimensional black holes where an appropriate index in the conformal field theory describing a system of branes and the macroscopic degeneracy computed from black hole entropy apparently differ at a subleading order $[39,40]$. In the context of certain five-dimensional black holes in M-theory on $K 3 \times T^{2}$ and $T^{6}$ even the leading asymptotics of the microscopic index apparently disagrees with the black hole entropy since the microscopic index vanishes [41]. One can remedy the situation in some cases by considering a modified index as suggested in [31, 42-45]. However, there are examples such as the one-sixteenth BPS black hole in $A d S_{5}$ where no microscopic index appears to have the right asymptotic growth that agrees with the black hole entropy [4650]. It is thus desirable both conceptually and practically to develop clear physical criteria for deciding when the black hole degeneracy is captured by a microscopic index and which particular index is relevant under what conditions.

An argument based on the symmetries of the near horizon geometry of the black hole was suggested in [38]. The basic idea is to use the black hole degeneracy as an input to compute an index on the macroscopic side and then compare this with the index computed on the microscopic side. This relies on the existence of an $A d S_{2}$ factor in the near horizon geometry of extremal black holes. The natural boundary condition on the various fields in $A d S_{2}$ is such as to fix all the charges (including angular momentum) and let the dual chemical potentials fluctuate. In particular a spherically symmetric horizon, being invariant under rotation, will represent an ensemble of states all of which carry zero angular momentum. Thus if $J$ denotes the third component of the angular momentum, and we define an index with the weight factor $(-1)^{F}:=\exp (2 \pi i J)$, then all the states which account for the entropy associated with the horizon will have $(-1)^{F}=1$ and hence

$$
\operatorname{Tr}(-1)^{F}=\operatorname{Tr}(1) .
$$

Furthermore, if the black hole preserves at least four supersymmetries, then spherical symmetry is forced on us since the closure of the symmetry algebra implies that the supergroup 
of symmetries is $\mathrm{SU}(1,1 \mid 2)$. This contains an $\mathrm{SU}(2)$ factor which can be identified with a subgroup of spatial rotations. Thus for such black holes (1.1) holds and the index equals the degeneracy.

This general argument needs to be further supplemented by taking into account the possible contribution from degrees of freedom living outside the horizon, - the hair modes $[51,52]$. These include in particular the fermion zero modes associated with the broken supersymmetry generators which account for the supermultiplet structure of a BPS state. The end result of this analysis expresses an appropriate index (helicity trace index) for the full black hole as the product of the degeneracy associated with the horizon (or horizons in case of multi-centered black holes) and the same helicity trace index for the hair degrees of freedom [38]. Since the contribution from the hair modes is usually small this explians why the black hole entropy represents the logarithm of an index to leading order. But this argument also tells us that at the subleading order we must take into account the effect of the hair modes while comparing the black hole entropy with the logarithm of the microscopic index. Indeed, without the hair modes one runs into internal inconsistencies when two different black hole solutions have identical near horizon geometries [51, 52].

The above line of argument thus gives us a precise route for computing an index from the macroscopic viewpoint which can then be compared with the microscopic results for the same index. However, explicit computation of the index on the macroscopic side is often quite challenging for two reasons. First, computing the entropy associated with the horizon requires us to carry out a path integral over the string fields in the near horizon geometry of the black hole. Second this procedure requires us to explicitly identify the hair modes by analyzing supersymmetric deformations of the (multi-) black hole solution and then quantizing them. These difficulties have been overcome in special cases in various approximations, often leading to non-trivial agreement between the macroscopic and microscopic results not only at the perturbative level $[2,6,11]$ but also at the non-perturbative level $[29,38,53-56]$. Furthermore this formalism also predicts correctly the sign of the index from the macroscopic side which agrees with the results of the microscopic analysis in a wide class of theories $[38,57]$.

In this paper we develop an alternative line of argument for computing the index on the macroscopic side in a special limit when only one of the charges carried by the black hole, representing momentum along an internal circle $S^{1}$ in some duality frame, becomes large. Even though this does not allow us to access the most general charge configuration, it provides a practical method for an exact computation for sufficiently general configurations for which all charges except the momentum can take any finite value. Moreover, by changing duality frames, one can choose different charges to play the role of the momentum that is becoming large and thus explore different regions of the charge lattice.

In the limit described above, the near horizon geometry of the black hole coincides with the near horizon geometry of an extremal BTZ black hole times a compact internal space $\mathcal{K}$ [58-60]. Furthermore, by taking the limit in which the asymptotic radius of $S^{1}$ approaches infinity, we can ensure that the full black hole geometry has an intermediate region where the space-time has the form of $A d S_{3} \times \mathcal{K}$, and the near horizon geometry is 
embedded in this geometry as an extremal BTZ black hole [61]. In this case, up to some additional contributions described below, the degeneracy associated with this black hole can be regarded as the degeneracy of states in the $C F T_{2}$ dual to the $A d S_{3}$, and in the limit of large momentum along $S^{1}$ this is given by the Cardy formula. Thus computation of the degeneracy reduces to the computation of the central charge of the dual $C F T_{2}$, which, as will be reviewed below, can be computed in terms of coefficients of the Chern-Simons term in the action of the bulk theory [60]. ${ }^{1}$ Note that this degeneracy includes the contribution from the black hole horizon, any hair modes which live outside the black hole horizon but inside the asymptotic $A d S_{3} \times \mathcal{K}$ geometry, and also multi-centered black hole configurations in $A d S_{3}$ (if they exist). This is not a problem since these must be included in the counting of states anyway. On the other hand this does not include the contribution from any modes which might live at the boundary of $A d S_{3} \times \mathcal{K}$ or between $A d S_{3} \times \mathcal{K}$ and the asymptotic space-time. By an abuse of notation we shall call these the exterior modes, - these will include for example the analog of the U(1) gauge fields for string theory in $A d S_{5} \times S^{5}[64$ 67]. Thus the contribution from these exterior modes need to be computed explicitly and combined with the $C F T_{2}$ contribution to get the full microscopic degeneracy.

Let us now turn to the computation of the index in the macroscopic theory. For this we first need to know which index in we should calculate. In order that we can compare the macroscopic results with the microscopic results it is important that we begin with an index whose definition does not require any prior knowledge of either the macroscopic geometry or the microscopic description of the system, but only on the charges and angular momenta of the state which can be measured unambiguously by an asymptotic observer. We shall call such an index a space-time index. In order that the index can be reliably computed on both sides we need to pick an appropriate space-time index which receives contribution from the BPS states under consideration but not from non-BPS states. In four dimensions this involves computing appropriate helicity supertraces [42-44] whereas in five dimensions one can use a slightly different version described e.g. in [31]. In either case this index involves computing a trace of $P$ multiplied by some polynomial in the angular momenta over states carrying a fixed set of charges, where $P$ - the analog of $(-1)^{F}$ for the Witten index - is a $\mathbb{Z}_{2}$ symmetry generator under which the unbroken supersymmetry generators have odd parity. The role of the angular momentum factor is to soak up the fermion zero modes arising from the $P$-odd broken supersymmetries. In the macroscopic description the contribution to this index comes from two separate sources: the bulk of $A d S_{3}$ and the exterior modes. By carefully analyzing the traces over these modes, and taking into account the fact that the fermion zero modes arising from the $P$-odd broken supersymmetries are part of the exterior modes, one finds that the full index involves a trace of $P$ in the $C F T_{2}$ dual to the bulk of $A d S_{3}$ and the trace of $P$ together with the angular momentum factors over the exterior modes.

For black holes which preserve at least four supercharges, the $A d S_{3}$ background that appears in the intermediate region has at least $(0,4)$ supersymmetry in the associated

\footnotetext{
${ }^{1}$ Although we are using the language of the holographically dual $C F T_{2}$, the computation is based on macroscopic analysis since the central charge is calculated from the effective action rather than from a microscopic calculation. This is also reinforced by the fact that for BTZ black holes Wald's formula [36] for the entropy takes the form of Cardy formula [60, 62, 63].
} 
supergravity theory. Thus the dual $C F T_{2}$ is actually a $(0,4)$ superconformal theory with an $\mathrm{SU}(2) \mathrm{R}$-symmetry group. Furthermore this R-symmetry group can be identified with the spatial rotation group or one of its subgroups. One finds that the operator $P$ restricted to this $C F T_{2}$ can be identified as $\operatorname{Tr}\left((-1)^{2 J_{R}}\right)$ where $J_{R}$ denotes the generator of the $\mathrm{U}(1)$ subgroup of $\mathrm{SU}(2)_{R}$. Thus the relevant $C F T_{2}$ index that appears in the expression for the space-time index is $\operatorname{Tr}\left((-1)^{2 J_{R}}\right)$, with the trace taken over the Ramond sector states of the $C F T_{2}$ carrying different values of $J_{R}$ but fixed values of $\left(L_{0}-\bar{L}_{0}\right) \equiv p$, and fixed values of all the $\mathrm{U}(1)$ charges associated with left-moving currents. This index receives non-vanishing contribution only from the Ramond sector ground states of the right-moving excitations of the $C F T_{2}$, i.e. only from states with $\bar{L}_{0}=0, L_{0}=p$. Now in the absence of the $(-1)^{2 J_{R}}$ insertion in the trace the large $p$ behaviour of this index is given by the Cardy formula and is determined by the left-moving Virasoro central charge $c_{L}$ as well as the levels of various left-moving $\mathrm{U}(1)$ current algebras under which the state carries charges. We shall argue in section 2 that the insertion of $(-1)^{2 J_{R}}$ does not change this behaviour since the effect of $(-1)^{2 J_{R}}$ under a modular transformation is to introduce a twist on the right-movers but does not affect the left-moving ground state. Thus the contribution to the index from the $\mathrm{CFT}_{2}$ is given by the Cardy formula. Combining this with the contribution from the exterior modes we can then recover the full macroscopic index.

While this gives a procedure for computing the index, the explicit computation still suffers from various technical complications. First of all in this approach we need to identify the exterior modes and compute their contribution to the index explicity. Furthermore to compute the contribution to the index from the bulk of $A d S_{3}$ we need the central charge and the levels of the U(1) current algebra. While these can be related to the coefficients of various Chern-Simons terms in the intermediate geometry that contains the $A d S_{3}$ factor, we still need to compute these coefficients after taking into account the effect of higher derivative and quantum corrections. There is however a further simplification that allows us to calculate the total index directly without having to compute separately the exterior and the bulk contribution. We shall argue that when one combines the contribution to the index from the bulk of $A d S_{3}$ and the exterior modes to compute the total index, the result is determined in terms of coefficients of Chern-Simons terms computed in the asymptotic space-time in which the black hole is embedded instead of in the intermediate geometry containing the $A d S_{3}$ factor. The former can be calculated explicitly, yielding an exact expression for the total contribution to the index in the $p \rightarrow \infty$ limit. Note that if instead of computing the index we had been computing the degeneracy, then no such simplification occurs, and we really need to compute separately the contribution from the bulk and the exterior modes and combine them to get the full result.

Armed with this result, we carry out explicit computation of the macroscopic results for the space-time index for four and five dimensional black holes in type IIB string theory compactified on $K 3 \times T^{2}, T^{6}, K 3 \times S^{1}$ and $T^{5}$ in different limits in which only one of the charges becomes large keeping the other charges fixed. We then compute the same space-time index on the microscopic side and compare this with the macroscopic results. For the microscopic computation we use two different techniques: we can begin with the exact formula for the index in string theories with 16 or 32 unbroken supersymmetries and 


\begin{tabular}{|c|c|c|c|}
\hline $\mathcal{M}$ & Limit & $\log d_{\text {macro }}$ & $\log d_{\text {micro }}$ \\
\hline$K 3$ & $\begin{array}{c}\text { Type-IIB } \\
\text { Cardy }\end{array}$ & $2 \pi \sqrt{Q_{1} Q_{5}\left(n-\frac{J^{2}}{4 Q_{1} Q_{5}}\right)}$ & $2 \pi \sqrt{Q_{1} Q_{5}\left(n-\frac{J^{2}}{4 Q_{1} Q_{5}}\right)}$ \\
\hline$K 3$ & $\begin{array}{c}\text { Type IIA } \\
\text { Cardy }\end{array}$ & $2 \pi \sqrt{Q_{5}(n+3)\left(Q_{1}-\frac{J^{2}}{Q_{5}(n-1)}\right)}$ & $2 \pi \sqrt{Q_{5}(n+3)\left(Q_{1}-\frac{J^{2}}{Q_{5}(n-1)}\right)}$ \\
\hline$T^{4}$ & $\begin{array}{c}\text { Type-IIB } \\
\text { Cardy }\end{array}$ & $2 \pi \sqrt{Q_{1} Q_{5}\left(n-\frac{J^{2}}{4 Q_{1} Q_{5}}\right)}$ & $2 \pi \sqrt{Q_{1} Q_{5}\left(n-\frac{J^{2}}{4 Q_{1} Q_{5}}\right)}$ \\
\hline
\end{tabular}

Table 1. Results for five-dimensional black holes for Type-IIB compactification on $\mathcal{M} \times S^{1}$.

study its limit when one of the charges becomes large, or we can represent the microscopic system as a configuration of M5-brane wrapped on $P \times S^{1}$ where $P$ and $S^{1}$ are appropriate four and one cycles of the compact space and then calculate its index in the limit of large momentum along $S^{1}$ using a Cardy like formula. Note that in the latter approach we need to use a generalization of the Cardy formula that determines the growth of the index rather than the degeneracy. In all cases, we find that the macroscopic prediction for the index always agrees with the microscopic index in the large momentum limit even for finite values of the other charges.

The results of our analysis are summarized in tables 1 and 2. In these tables $d_{\text {macro }}$ denotes the macroscopic result for the appropriate space-time index and $d_{\text {micro }}$ denotes the result of microscopic computation of the same space-time index. Below we give more detailed explanation of the various entries in these tables.

- Five-dimensional black holes table 1 shows the results for spinning five-dimensional black holes in Type-IIB string theory compactified on $\mathcal{M} \times S^{1}$, carrying $Q_{5}$ units of D5-brane charge wrapped on $\mathcal{M} \times S^{1}, Q_{1}$ units of D1-rane charge wrapped on $S^{1}$, momentum $n$ along $S^{1}$ and angular momentum $J$. The second column of this table contains information about the limits we consider and the frame that we use for computing $d_{\text {macro }}$ in these limits. In particular while in the Type-IIB Cardy limit $\left(n-\frac{J^{2}}{4 Q_{1} Q_{5}}\right) \rightarrow \infty$, we carry out the macroscopic computation directly in the type IIB frame, in the Type-IIA Cardy limit $\left(Q_{1}-\frac{J^{2}}{Q_{5}(n-1)}\right) \rightarrow \infty$, we need to go to a dual type IIA frame where $Q_{1}$ appears as the momentum.

- Four-dimensional black holes table 2 shows the results for four-dimensional nonspinning black holes in M-theory compactified on $\mathcal{M} \times T^{2} \times S^{1}$, carrying $Q_{1}$ units of M5-brane charge wrapped on $C_{2} \times T^{2} \times S^{1}, Q_{5}$ units of M5-brane charge wrapped on $\widetilde{C}_{2} \times T^{2} \times S^{1}, K$ units of M5-brane charge wrapped on $\mathcal{M} \times S^{1}$ and $n$ units of momentum along $S^{1}$. Here $C_{2}$ and $\widetilde{C}_{2}$ denote a pair of dual 2-cycles of $\mathcal{M}$. The limit we consider is $n \rightarrow \infty$ which corresponds to taking the $L_{0}$ eigenvalue large in the boundary $\mathrm{CFT}_{2}$.

The results in both tables clearly show that the macroscopic prediction $d_{\text {macro }}$ for the space-time index always agrees with the microscopic prediction $d_{\text {micro }}$ for the same index. 


\begin{tabular}{|c|c|c|}
\hline $\mathcal{M}$ & $\log d_{\text {macro }}$ & $\log d_{\text {micro }}$ \\
\hline$K 3$ & $2 \pi \sqrt{\left(Q_{1} Q_{5} K+4 K\right) n}$ & $2 \pi \sqrt{\left(Q_{1} Q_{5} K+4 K\right) n}$ \\
\hline$T^{4}$ & $2 \pi \sqrt{\left(Q_{1} Q_{5} K\right) n}$ & $2 \pi \sqrt{\left(Q_{1} Q_{5} K\right) n}$ \\
\hline
\end{tabular}

Table 2. Results for four-dimensional black holes for M-theory compactified on $\mathcal{M} \times T^{2} \times S^{1}$.

There are several novelties in our analysis which are worth emphasizing:

1. The formulæ quoted in the two tables are exact in the limits mentioned, i.e. they hold even when the charges other than the one which is taken to infinity are finite. Thus, they go far beyond the supergravity approximation and incorporate the effects of $\alpha^{\prime}$ and string loop corrections. On the macroscopic side this is achieved by an exact computation of the coefficients of certain Chern-Simons terms in the action whereas on the microscopic side this is achieved by the use of an exact microscopic formula for the index evaluated in the same limits as described above.

2. In all cases, the limits that we consider can be regarded as a Cardy limit of a $\mathrm{CFT}_{2}$ in an appropriate duality frame. If the underlying $\mathrm{CFT}_{2}$ is weakly coupled in this duality frame, we can calculate $d_{\text {micro }}$ with the help of the Cardy like formula for the index and degeneracy. This is the case for the Type-IIB Cardy limit in table 1. However in some cases, the microscopic configuration may contain a set of NS5-branes and as a result, a weakly coupled description of the $\mathrm{CFT}_{2}$ may not be available. This is the case for the type IIA Cardy limit in table 1.

3. Since $d_{\text {micro }}$ is an index which does not change under duality, ${ }^{2}$ one might expect that $d_{\text {micro }}$ can always be computed in an appropriate duality frame where a weakly coupled $\mathrm{CFT}_{2}$ description is available. Indeed for all the examples in table 1, a weakly coupled $\mathrm{CFT}_{2}$ description is available in the Type-IIB frame, and this allows us to compute $d_{\text {micro }}$. However, under this duality, the type IIA Cardy limit corresponds to an 'anti-Cardy' limit ( $L_{0}$ eigenvalue fixed and $c$ large) in the Type-IIB frame. As a result, usual methods of asymptotic evaluations are not applicable. One can nevertheless compute the asymptotics in this limit from the exact formula using the methods of $[68,69]$ which cleverly exploit the additional symmetries of the exact counting function.

4. Our result for four dimensional black holes resolves a puzzle raised in [39, 40] involving black holes in M-theory compactified on $K 3 \times T^{3}$. A naive application of the results of [60] without accounting for the different treatment required for the $C F T_{2}$ dual to the bulk of $A d S_{3}$ and the exterior modes led to an apparent mismatch between black hole entropy and the logarithm of the microscopic degeneracy. For example, if one evaluates the absolute degeneracy in the microscopic theory at weak coupling, then one obtains $2 \pi \sqrt{\left(Q_{1} Q_{5} K+6 K\right) n}$ for the logarithm of the absolute degeneracy which differs from the correct macroscopic answer at sub-leading order. In contrast, our

\footnotetext{
${ }^{2}$ In general, the index can also jump because of wall-crossings but in the $\mathcal{N}=4$ context these are exponentially subleading corrections not relevant to the present analysis.
} 
analysis leads to a perfect agreement between the microscopic and the macroscopic results as shown in table 2. This example thus underscores the necessity and utility of defining a macroscopic supersymmetric index from black hole entropy for correct comparisons with microscopic computations.

5. Our analysis also gives explicit form of the entropy of five dimensional spinning black holes after taking into account the effect of higher derivative corrections. Previous attempts to do this involved using a specific set of higher derivative terms in the five dimensional effective action [70-72]. In contrast our analysis relies on the ability to express the entropy in terms of coefficients of certain Chern-Simons terms in the action, and is exact in the limit considered. This also agrees with the prediction from the microscopic side based on the exact formula for the index.

For M5 branes wrapped on $S^{1}$ times a four cycle of a generic Calabi-Yau manifold, ref. [60] presented an argument explaining why the microscopic and the macroscopic entropy would always agree in the Cardy limit. This argument was based on the observation that in a $(1+1)$ dimensional conformal field theory with $(0,4)$ world-sheet supersymmetry, the Virasoro central charge $c_{R}$ carried by the right movers is related to the level of the rightmoving SU(2) R-symmetry current. This in turn is related to the anomaly in this Rsymmetry current. Using anomaly inflow and identifying the SU(2) R-symmetry current as (a subgroup of) the spatial rotation one can relate this to the coefficient of the $\mathrm{SU}(2)$ Chern-Simons terms in the effective action. Furthermore the difference $c_{L}-c_{R}$ between the left- and right-moving central charges is related to the gravitational anomaly in the world-sheet theory of the brane system which in turn is related to the coefficient of the gravitational Chern-Simons term in the effective action of string theory. Using these one can express the central charge $c_{L}$ of the left-moving Virasoro algebra - which controls the growth of the microscopic degeneracy — in terms of the gravitational and SU(2) ChernSimons terms in the effective action. The latter in turn controls the black hole entropy, leading to the equality between the macroscopic and the microscopic entropy.

In our examples, the Calabi-Yau manifold is either $K 3 \times T^{2}$ or $T^{6}$. Since the systems we analyze also have four unbroken supersymmetries, it is natural to ask if similar argument can be used to explain the agreement between the microscopic and the macroscopic entropies in our systems. The main additional complication that arises in our case is the failure of the identification of the R-symmetry current of the microscopic theory with the spatial rotation group. We find that while for the part of the microscopic system that controls most of the entropy this identification is correct; it fails for a small component. ${ }^{3} \mathrm{~A}$ simple example of this is provided by the scalar modes representing transverse oscillation of the brane. These are non-chiral modes on the brane world-volume and transform in the $\left(2_{L}, 2_{R}\right)$ representation of the rotation group $\mathrm{SU}(2)_{L} \times \mathrm{SU}(2)_{R}$ in five dimensions and 3 representation of the rotation group $\mathrm{SU}(2)$ in four dimensions. For definiteness let us focus on the five dimensional case. In order to identify the $\mathrm{SU}(2)_{R}$ subgroup of the rotation

\footnotetext{
${ }^{3}$ A similar mismatch was found in [73] between the modes living on the Coulomb and the Higgs branch of the D1-D5 system. Here the disagreement is between different components of the CFT at the same point in the moduli space.
} 
group in five dimensions as the right-moving R-symmetry on the brane world-volume this must act trivially on the left-movers. This clearly fails for the left-moving part of the above scalars which transform in the fundamental representation of $\mathrm{SU}(2)_{R}$. As a result the total anomaly in the $\mathrm{SU}(2)_{R}$ spatial rotation symmetry is not related to the level of the $\mathrm{SU}(2)$ R-symmetry current in the world-sheet theory, and the growth of the degeneracy of the microscopic system is no longer controlled by the anomaly coefficients which can be directly related to the coefficients of the Chern-Simons terms in the effective action. A similar problem occurs in the macroscopic description. For the CFT that is holographically dual to the bulk of the $A d S_{3}$ factor appearing in the near horizon geometry, the R-symmetry can be identified as the spatial $\mathrm{SU}(2)_{R}$ rotational symmetry acting on the space transverse to $A d S_{3}$. But this identification need not hold for the exterior modes which might live on the boundary of $A d S_{3}$ - the analog of the U(1) super Yang-Mills theory for type IIB supergravity on $A d S_{5} \times S^{5}$ - or between $A d S_{3}$ and the asymptotic infinity. In particular these modes include the transverse oscillation modes of the brane which fail to satisfy the conditions needed for identifying the R-symmetry with spatial $\mathrm{SU}(2)_{R}$ rotation. For this reason the coefficients of the Chern-Simons terms in the effective action do not directly give us information about the growth of the degeneracy obtained by combining the black hole entropy with the contribution from these additional exterior modes. Remarkably however we find that the results on both sides simplifiy when we focus on an appropriate index rather than the absolute degeneracy. In the microscopic theory we find that the growth of the index is directly controlled by the gravitational and rotational anomaly coefficients exactly as they would have controlled the growth of the degeneracy if the subtle difference between the R-symmetry transformation and spatial rotation had been absent. On the macroscopic side we find that total contribution to the index from the black hole living in the bulk of $A d S_{3}$ and the exterior modes is controlled by the coefficients of the ChernSimons terms in the effective action in the asymptotic space-time in which the black hole is embedded. Since the latter are related to the anomaly coefficients in the microscopic theory this allows us to establish the equality between the microscopic and the macroscopic index.

The rest of the paper is organized as follows. In section 2 we review the argument relating the black hole entropy to an index, and give an alternative argument leading to similar results for special class of black holes whose near horizon geometry contains a locally $A d S_{3}$ factor. In section 3 we compute the macroscopic index of a class of spinning five dimensional black holes and non-spinning four dimensional black holes in appropriate limit in which the near horizon geometry develops an $A d S_{3}$ factor. In section 4 we complement the analysis of section 3 by including the effect of the exterior contribution to the macroscopic index. In section 5 we use the known expressions for the exact microscopic index of these systems to extract its behaviour in the various Cardy limits and find perfect agreement with the macroscopic results of section 3 and 4 . In section 6 we repeat the analysis of section 5 using the M-theory description for the four dimensional black holes. While in this description we cannot calculate the index exactly, we can compute it in the Cardy limit and find precise agreement with the results of section 5. Both in section 5 and section 6 we also calculate the microscopic degeneracy whenever there is an underlying two dimensional weakly coupled conformal field theory, and find that in some cases they differ from the microscopic values of the space-time index. In section 7 we give a general proof 
of why the microscopic and the macroscopic computation of the index must always agree. This argument is a generalization of the argument of [60] by taking into account existence of degrees of freedom for which the R-symmetry generators of the world-sheet theory do not always match with the spatial rotation generators - a fact that was crucial in the argument of [60]. This analysis also explains why the degeneracy and index do not always grow at the same rate. In appendix A we describe the computation of the coeffcients of the Chern-Simons terms which arise from dimensional reduction of gauge invariant Lagrangian density in higher dimensions. In appendix B we complement the analysis of asymptotic growth of the exact microscopic index in section 5 by demonstrating that some terms, which were ignored in the analysis of section 5 , are indeed small compared to the leading terms.

\section{Computing the index in the macroscopic theory}

In this section we first introduce the relevant indices for counting BPS states in four and five dimensional black holes and then review the argument of $[38,74]$ as to how the degeneracy of a supersymmetric black hole, computed by exponentiating the entropy, can be used to compute a macroscopic index that can be compared with a microscopic index. We then give an alternative version of this argument that applies to the special case of black holes with locally $A d S_{3}$ factors in their near horizon geometry.

We begin by defining the helicity trace index in four dimensions. Due to Lorentz invariance the number of supercharges in a four dimensional theory is always a multiple of 4; furthermore the number of supersymmetries preserved by a state is also a multiple of 4. If we consider a black hole that breaks altogether $4 k$ supercharges, then the standard index for counting these states is the helicity trace index $B_{2 k}$ defined as [42-44]

$$
B_{2 k}=\frac{1}{(2 k) !} \operatorname{Tr}\left[(-1)^{F}(2 h)^{2 k}\right]=\frac{1}{(2 k) !} \operatorname{Tr}\left[e^{2 \pi i h}(2 h)^{2 k}\right],
$$

where $h$ is the third component of the angular momentum of a state in the rest frame, and the trace is taken over all states carrying a given set of charges. In order that a given state gives a non-vanishing contribution to this index, the number of supersymmetries broken by the state must be less than or equal to $4 k$; otherwise trace over the fermion zero modes associated with the broken supersymmetries will make the trace vanish. On the other hand if we have states with precisely $4 k$ broken supersymmetries then $B_{2 k}$ receives contribution from these states, but not from any other state with more than $4 k$ broken supersymmetries. Since quantization of each pair of fermion zero modes produces a pair of states carrying $h= \pm \frac{1}{4}$, the trace over the $4 k$ fermion zero modes associated with the broken supersymmetries is given by

$$
\left(e^{i \pi / 2}-e^{-i \pi / 2}\right)^{2 k}(2 k) ! / 2^{2 k}=(-1)^{k}(2 k) ! .
$$

The $(2 k)$ ! term arises from the binomial expansion of $(2 h)^{2 k}$ after expressing $h$ as the sum of contributions from different pairs. This cancels the similar factor in the denominator in (2.1), leaving behind a contribution of $(-1)^{k}$.

It is easy to find a generalization of this in five dimensions. The spatial rotation group in five dimensions is $\mathrm{SU}(2)_{L} \times \mathrm{SU}(2)_{R}$. We shall denote by $J_{L}$ and $J_{R}$ their $\mathrm{U}(1)$ 
generators. Among the set of all the supersymmetry generators of the theory, half belong to $\left(2_{L}, 1_{R}\right)$ representation of $\mathrm{SU}(2)_{L} \times \mathrm{SU}(2)_{R}$ and the other half belong to the $\left(1_{L}, 2_{R}\right)$ representation of $\mathrm{SU}(2)_{L} \times \mathrm{SU}(2)_{R}$. For a state preserving 4 supersymmetries, the unbroken supersymmetry generators can be either in the $\left(2_{L}, 1_{R}\right)$ or in the $\left(1_{L}, 2_{R}\right)$ representation; we shall choose the convention in which they are in the $\left(1_{L}, 2_{R}\right)$ representation. The rest of the supersymmetry generators will be broken, giving rise to fermion zero modes carrying the quantum numbers of the broken generators. Let $4 k$ be the number of broken generators in the $\left(1_{L}, 2_{R}\right)$ representation. We now consider the index [31]

$$
C_{2 k} \equiv \frac{(-1)^{k}}{(2 k) !} \operatorname{Tr}\left[(-1)^{2 J_{R}}\left(2 J_{R}\right)^{2 k}\right]
$$

where the trace is taken over all states carrying a fixed value of $J_{L}$ and fixed set of charges but all possible values of $J_{R}$. Without the $\left(2 J_{R}\right)^{2 k}$ factor the trace over the $\left(1_{L}, 2_{R}\right)$ fermion zero modes carrying $\left(J_{L}, J_{R}\right)=\left(0, \pm \frac{1}{2}\right)$ would make the trace vanish. However the $\left(2 J_{R}\right)^{2 k}$ factor soaks up the $2 k$ pairs of fermion zero modes exactly as in the case of four dimensional black holes and gives a non-vanishing result. There are also $\left(2_{L}, 1_{R}\right)$ fermion zero modes carrying $\left(J_{L}, J_{R}\right)=\left( \pm \frac{1}{2}, 0\right)$, but they do not make the trace vanish since the trace is taken over states carrying a fixed $J_{L}$. It is also easy to see that the non-BPS states do not contribute to this index. They would have additional fermion zero modes in the $\left(1_{L}, 2_{R}\right)$ representation and hence trace over these fermion zero modes would make the index vanish.

As an example, we can consider the BMPV black hole [75] in type IIB string theory compactified on $K 3 \times S^{1}$. This breaks 12 out of 16 supersymmetries. Eight of the broken supersymmetry generators are in the $\left(2_{L}, 1_{R}\right)$ representation, four of the broken generators are in the $\left(1_{L}, 2_{R}\right)$ representation and the four unbroken generators are in the $\left(1_{L}, 2_{R}\right)$ representation. Since there are four broken generators in the $\left(1_{L}, 2_{R}\right)$ representation the argument given above shows that the relevant index is $C_{2}$. Similarly if we consider BMPV black hole in type IIB string theory on $T^{4} \times S^{1}$ then it breaks 28 of the 32 supersymmetries, with 16 broken generators in the $\left(2_{L}, 1_{R}\right)$ representation, 12 broken generators in the $\left(1_{L}, 2_{R}\right)$ representation and 4 unbroken generators in the $\left(1_{L}, 2_{R}\right)$ representation. The index required for counting these states is $C_{6}$.

Let us now compute the contribution to these indices from BPS black holes with four supercharges. For definiteness we begin with a four dimensional black hole breaking $4 k$ supersymmetries and compute the index $B_{2 k}$. The net contribution to the index from a black hole can be expressed as a sum of products of the contributions from the horizon and the hair [38, 51, 52]; this could involve contribution from multiple horizons for multicentered black holes. Let us first focus on the contribution from single centered black holes. Since the fermion zero modes associated with broken supersymmetries live outside the horizon and hence are part of the hair degrees of freedom of the black hole $[51,52],{ }^{4}$

\footnotetext{
${ }^{4}$ The fermion zero mode associated with a broken supersymmetry generator can be constructed as follows. We make a supersymmetry transformation of the original solution by an infinitesimal parameter that approaches a constant spinor corresponding to the broken generator at infinity and vanishes for $r<a$ for some constant $a$. By choosing $a$ such that the horizon lies at $r<a$ we can ensure that such deformations live outside the horizon and hence are part of the hair degrees of freedom.
} 
we can express the contribution to the index from the black hole as

$$
B_{2 k}=\frac{1}{(2 k) !}\left[\operatorname{Tr}_{\text {hor }}(-1)^{2 h_{\text {hor }}}\right]\left[\operatorname{Tr}_{\text {hair }}(-1)^{2 h_{\text {hair }}}\left(2 h_{\text {hair }}\right)^{2 k}\right],
$$

where $h_{\text {hor }}$ and $h_{\text {hair }}$ denote the helicities carried by the hair and the horizon. For states carrying a fixed set of charges $\vec{q}$ this can be expressed as

$$
B_{2 k}(\vec{q})=\sum_{\vec{q}_{h o r}} B_{0 ; h o r}\left(\vec{q}_{h o r}\right) B_{2 k ; \text { hair }}\left(\vec{q}-\vec{q}_{h o r}\right),
$$

where

$$
B_{0 ; h o r}(\vec{q})=\operatorname{Tr}_{h o r ; \vec{q}}(-1)^{2 h_{h o r}}
$$

and

$$
B_{2 k ; \text { hair }}(\vec{q})=\operatorname{Tr}_{\text {hair } ; \vec{q}}(-1)^{2 h_{\text {hair }}}\left(2 h_{\text {hair }}\right)^{2 k} .
$$

Here $\vec{q}$ in the subscript of $\operatorname{Tr}$ denotes that the trace is being taken over states carrying a fixed set of charges $\vec{q}$. We now argue that if the black hole has 4 unbroken supersymmetries and if its near horizon geometry has an $A d S_{2}$ factor, then it must carry $h_{\text {hor }}=0$. The argument goes as follows. The closure of the $\mathrm{SL}(2, \mathbb{R})$ isometry of the near horizon geometry, and the unbroken supersymmetries requires that the near horizon geometry has the full $s u(1,1 \mid 2)$ symmetry algebra. This includes $s u(2)$ as a subalgebra, forcing the horizon to be spherically symmetric and hence carry zero angular momentum. ${ }^{5}$ This gives

$$
B_{0 ; h o r}(\vec{q})=\operatorname{Tr}_{h o r ; \vec{q}}(-1)^{2 h_{h o r}}=\operatorname{Tr}_{h o r ;} \vec{q}(1)=d_{h o r}(\vec{q})
$$

where $d_{\text {hor }}(\vec{q})$ is the degeneracy associated with the horizon degrees of freedom for charge $\vec{q}$. In the classical limit it is given by the exponential of the Wald entropy, but more generally it can be computed from the path integral over the string fields in the near horizon geometry [37]. Using (2.5) and (2.8) we get the contribution to $B_{2 k}$ from the black hole

$$
B_{2 k}(\vec{q})=\sum_{\vec{q}_{h o r}} d_{h o r}\left(\vec{q}_{h o r}\right) B_{2 k ; h a i r}\left(\vec{q}-\vec{q}_{\text {hair }}\right) .
$$

$B_{2 k ; \text { hair }}(\vec{q})$ can be computed once we have identified the hair degrees of freedom of the black hole. Thus (2.9) can be used to make a prediction for the index $B_{2 k}(\vec{q})$ from the macroscopic side. Note also that since $d_{h o r}(\vec{q})$ is positive $(2.9)$ makes a definite prediction for the sign of $B_{2 k}$ provided we have sufficient knowledge of $B_{2 k ; \text { hair }}$. In particular in situations where the only hair modes are the fermion zero modes associated with broken supersymmetries, we have $\vec{q}_{\text {hair }}=0, B_{2 k ; \text { hair }}=(-1)^{k}$ and hence $(-1)^{k} B_{2 k}=d_{\text {hor }}>0$. As

\footnotetext{
${ }^{5}$ In asymptotically Minkowski space-time or $A d S_{d}$ space-time with $d \geq 4$, where the asymptotic boundary conditions are set by the chemical potentials instead of the charges, the spherical symmetry of the background will correspond to evaluating the partition function at zero value of the chemical potential conjugate to the angular momentum. However the path integral over the string fields in the near horizon $A d S_{2}$ geometry that is used to compute the horizon degeneracy must be carried out over configurations carrying fixed values of the total charges including angular momentum [37, 76]. Thus in this case spherical symmetry implies zero value of the angular momentum carried by the black hole.
} 
was shown in $[38,57]$, the macroscopic prediction for the sign of $B_{6}$ agrees with the result of explicit microscopic computation for all the $\mathcal{N}=4$ supersymmetric string theories for which this index has been computed. The generalization of (2.9) to multi-centered black holes is straightforward; since each center carries zero angular momentum due to supersymmetry, the contribution to $B_{2 k}$ will be given by a formula analogous to (2.9), with $d_{h o r}$ replaced by the product of $d_{h o r}$ from each center and we have to sum over all possible ways of distributing the total charge among the horizon and the hair.

This argument has a straightforward generalization to five dimensions with $h$ replaced by $J_{R}$. Incidentally, this reasoning also implies the well-known facts that the horizon of a supersymmetric black hole cannot carry any spin in four dimensions, and that the horizon of a supersymmetric black hole can carry only the $\mathrm{SU}(2)_{L}$ spin in five dimensions. Also this argument does not generalize to the problematic one-sixteenth BPS black holes in $A d S_{5}$ since they have too little supersymmetry, and the completion of the algebra containing the supersymmetry generators and the $\mathrm{SL}(2, \mathbb{R})$ isometry of $A d S_{2}$ do not force us to have an $\mathrm{SU}(2)$ symmetry in the near horizon geometry.

While this argument explains the relation between the index and degeneracy, applying this argument to compute the contribution to the index from the macroscopic side requires identifying explicitly the hair modes of the black hole which is not always an easy task [51, 52]. Also this would require computing $d_{h o r}$ by evaluating the path integral over string fields in the near horizon background geometry [37] - another difficult problem. For these reasons we shall now give an alternative approach to computing the index on the macroscopic side which is in the same spirit but differs in details. If we consider a black hole for which one of the charges can be identified as an internal momentum along some circle $S^{1}$, and if we consider a limit in which this momentum becomes large keeping all the other charges fixed, then the near horizon geometry of such a black hole is known to develop a locally $A d S_{3}$ factor by combining the near horizon $A d S_{2}$ geometry with this internal circle $S^{1}[58,59]$. Furthermore if we now adjust the asymptotic moduli fields in such a way that we take the asymptotic value of the radius of $S^{1}$ to infinity keeping all the other moduli fixed, then the solution also develops a global $A d S_{3}$ factor in the intermediate region, and the black hole solution can now be regarded as the BTZ black hole living in this asymptotically $A d S_{3}$ space-time $[61,77,78]$. The classical entropy of this black hole has the form of a Cardy formula, with the central charge given by some specific function of the parameters of the Lagrangian $[60,62,63]$. Thus the classical black hole entropy can be reinterpreted as the Cardy formula of the $\mathrm{CFT}_{2}$ that is holographically dual to string theory in this geometry. Since the Cardy formula in $\mathrm{CFT}_{2}$ is expected to hold in the full quantum theory this suggests that we can use Cardy formula as the quantum generalization of the black hole entropy. The problem of computing the quantum corrected entropy of the black hole then reduces to the problem of computing the quantum corrected central charge. Since we do not have direct knowledge of the $\mathrm{CFT}_{2}$, this has to be computed using the data in the bulk theory after taking into account quantum corrections to the bulk effective action. In this sense the entropy computed this way is still the macroscopic entropy.

There are however several subtleties overlooked in the above discussion. First of all the Cardy formula is supposed to count total degeneracy of states in $C F T_{2}$ without caring 
about whether they are represented as single or multicentered black holes inside $A d S_{3}$, or whether the contribution comes from the horizon or the hair modes. So the above definition of the black hole entropy includes all of these contributions. This is not a serious problem since in order to compare the macroscopic result with the microscopic result we need to sum over all the contributions on the macroscopic side in any case. The microscopic degeneracy may also receive contribution from configurations with multiple $A d S_{3}$ throat [61], but this can be avoided by working in appropriate domains in the moduli space. In any case in theories with 16 or more supercharges the contribution from the multicentered black holes is small and we shall ignore their contribution in our analysis. The main complication arises from the fact that the degeneracy of the $\mathrm{CFT}_{2}$ dual to the theory living on the bulk of $A d S_{3}$ does not capture all the degrees of freedom of the system. There may be additional degrees of freedom living on the boundary of $A d S_{3}$ (analogous to the $\mathrm{U}(1)$ factor for $A d S_{5}$ [79]), or in the region between $A d S_{3}$ and the asymptotic infinity. This will in particular include the Goldstino fermion zero modes associated with supersymmetries which are broken by the $A d S_{3}$ background. We shall collectively call all such modes exterior modes. ${ }^{6}$ Since in the limit we are considering — taking the asymptotic radius of $S^{1}$ to infinity keeping the momentum quantum number fixed - the physical momentum vanishes, part of the black hole solution lying between the asymptotic space-time and the intermediate $A d S_{3}$ region has full $1+1$ dimensional Lorentz symmetry. Thus we would expect the dynamics of the exterior modes to be described by some $(1+1)$ dimensional field theory. Their contribution has to be combined with the Cardy formula to recover the total degeneracy of states.

So far we have talked about degeneracy, but our real interest is in the index. Let us now see how the above discussion will change when we try to compute the index instead of the degeneracy. Again for definiteness we shall first consider four dimensional black holes and compute the index $B_{2 k}$. Denoting by $h_{b u l k}$ and $h_{\text {exterior }}$ the contribution to $h$ from the degrees of freedom living in the bulk and the exterior of $A d S_{3}$, we can express the trace appearing in (2.1) as

$$
B_{2 k}=\frac{1}{(2 k) !} \operatorname{Tr}\left[e^{2 \pi i\left(h_{\text {bulk }}+h_{\text {exterior }}\right)}\left(2 h_{\text {bulk }}+2 h_{\text {exterior }}\right)^{2 k}\right]
$$

For simplicity we shall assume that the supersymmetries broken by the black hole are also broken by the intermediate $A d S_{3}$ region, i.e. the black hole, when regarded as a solution in

\footnotetext{
${ }^{6}$ The need for separating out the exterior modes can be seen as follows. In the microscopic theory where the dynamics is described by that of an oscillating string there are a set of degrees of freedom associated with the center of mass motion which are decoupled from the rest of the degrees of freedom. This decoupling in the infrared limit follows from Goldstone's theorem and is expected to be exact even in the full interacting theory. Thus if the $C F T_{2}$ dual to $A d S_{3}$ had contained the full set of degrees of freedom of the black hole then this CFT will be given by a sum of two (or more) CFT's which do not interact with each other. Thus we can define two stress tensors and hence there must be two gravitons in the bulk theory, in contradiction to what we see. Furthermore in the bulk theory the SU(2) R-symmetry group of $(0,4)$ supersymmetry can be directly related to the spatial rotation group for four dimensional black holes and the $\mathrm{SU}(2)_{R}$ subgroup of the spatial rotation group for five dimensional black holes. This identification fails to hold for the CFT containing the center of mass modes, showing again that these modes must live outside the bulk of $A d S_{3}$.
} 
$A d S_{3}$, does not break any further supersymmetry. ${ }^{7}$ In this case all the fermion zero modes associated with broken supersymmetry are part of the exterior degrees of freedom, and in order to get a non-vanishing contribution to the trace in (2.10) we need to pick the factor of $\left(2 h_{\text {exterior }}\right)^{2 k}$ from the binomial expansion of $\left(2 h_{\text {bulk }}+2 h_{\text {exterior }}\right)^{2 k}$. This gives

$$
B_{2 k}=\frac{1}{(2 k) !} \operatorname{Tr}\left[e^{2 \pi i\left(h_{\text {bulk }}+h_{\text {exterior }}\right)}\left(2 h_{\text {exterior }}\right)^{2 k}\right]=\sum_{\vec{q}} B_{\text {bulk }}\left(\vec{q}_{\text {bulk }}\right) B_{2 k ; \text { exterior }}\left(\vec{q}-\vec{q}_{\text {bulk }}\right),
$$

where $B_{b u l k}=T r_{b u l k} e^{2 \pi i h_{b u l k}}$ in a fixed charge sector.

In the Cardy limit one of the charges, which we shall call $p$, becomes large. We shall denote by $\vec{Q}$ the rest of the charges and denote by $\sim$ the Fourier transform of various quantities $B_{2 k}, B_{2 k ; \text { exterior }}$ etc. with respect to the charge $p$. For example

$$
\widetilde{B}_{2 k}(\vec{Q}, \tau)=\sum_{p} B_{2 k}(\vec{Q}, p) e^{2 \pi i p \tau},
$$

etc. We shall now make the assumption that the exterior modes do not carry any charge other than $p$, so that in the sum in $(2.11) \vec{Q}_{b u l k}$ is always equal to $\vec{Q}$. Then $(2.11)$ takes the form:

$$
\widetilde{B}_{2 k}(\vec{Q}, \tau)=\widetilde{B}_{b u l k}(\vec{Q}, \tau) \widetilde{B}_{2 k ; \text { exterior }}(\tau) .
$$

Our goal is to compute the behaviour of $B_{2 k}(\vec{Q}, p)$ for large $p$. This is controlled by the behaviour of $\widetilde{B}_{2 k}(\vec{Q}, \tau)$ for small $\tau$. To determine this we need to find the small $\tau$ behaviour of $\widetilde{B}_{b u l k}(\vec{Q}, \tau)$ and $\widetilde{B}_{2 k ; \text { exterior }}(\tau)$. First we focus on $\widetilde{B}_{b u l k}(\vec{Q}, \tau)$. If instead of the index $B_{b u l k}(\vec{Q}, p)$ we had been interested in the degeneracy $d_{b u l k}(\vec{Q}, p) \equiv \operatorname{Tr}(1)$ of left-moving excitations in the $\mathrm{CFT}_{2}$, then for large $p$ it would grow as $\exp \left[2 \pi \sqrt{c_{L}^{b u l k} p / 6}\right]$ according to the Cardy formula, where $c_{L}^{b u l k}$ is the central charge of the left-moving Virasoro algebra of the $\mathrm{CFT}_{2}$. This implies

$$
\widetilde{d}_{\text {bulk }}(\vec{Q}, \tau) \sim \exp \left[\pi i c_{L}^{b u l k} / 12 \tau\right],
$$

for small $\tau$. We shall now argue that for small $\tau$ the behaviour of $\widetilde{B}_{b u l k}(\vec{Q}, \tau)$ is given by the same formula. The argument goes as follows. With the help of a modular transformation in the two dimensional CFT, the behaviour of $d_{b u l k}$ in the Cardy limit can be related to the ground state energy of the left-moving sector, and this is what leads to (2.14), with $-c_{L}^{\text {bulk }} / 24$ interpreted as the ground state energy of the left-moving sector. Now if instead of $\widetilde{d}_{b u l k}$ we consider the index $\widetilde{B}_{\text {bulk }}$, then following the same logic we can relate its small $\tau$ behaviour to the ground state energy in the left-moving sector, but this time with a $(-1)^{2 h_{\text {bulk }}}$ twisted boundary condition under $\sigma \rightarrow \sigma+2 \pi, \sigma$ being the world-sheet space coordinate. Now quite generally when the black hole (and the associated $A d S_{3}$ ) has four unbroken supersymmetry generators, they combine with the conformal symmetry of the $A d S_{3}$ to generate a $(0,4)$ superconformal algebra. This includes an SU(2) R-symmetry current whose global part can be identified as the spatial rotation symmetry. Due to this

\footnotetext{
${ }^{7}$ In some cases the unbroken supersymmetry generators get modified when we switch on the charges on the black hole, e.g. when we switch on M2-brane charges on an M5-brane [31, 80]. For the systems we shall analyze this does not happen.
} 
identification, $h_{\text {bulk }}$ can be interpreted as the zero mode of the $\mathrm{U}(1) \subset \mathrm{SU}(2)$ R-symmetry current of the $\mathrm{CFT}_{2}$. Since the twist by the zero mode of the right-moving $\mathrm{U}(1) \subset \mathrm{SU}(2)$ $\mathrm{R}$-symmetry current of the $\mathrm{CFT}_{2}$ is not expected to affect the ground state energy in the left-moving sector, this energy will continue to be given by $-c_{L}^{b u l k} / 24$, and hence the small $\tau$ behaviour of $\widetilde{B}_{b u l k}$ is also given by the Cardy formula:

$$
\widetilde{B}_{b u l k}(\vec{Q}, \tau) \sim \exp \left[\pi i c_{L}^{b u l k} / 12 \tau\right] .
$$

We shall see in section 4 that for small $\tau \widetilde{B}_{2 k \text {;exterior }}(\overrightarrow{0}, \tau)$ is given by a formula similar to $(2.15):^{8}$

$$
\widetilde{B}_{2 k ; \text { exterior }}(\overrightarrow{0}, \tau) \sim \exp \left[\pi i c_{L, \text { eff }}^{\text {exterior }} / 12 \tau\right],
$$

for some constant $c_{L, e f f}^{\text {exterior }}$. Substituting (2.14) and (2.16) into (2.13) we get

$$
\widetilde{B}_{2 k}(\vec{Q}, \tau) \sim \exp \left[\pi i c_{L, e f f}^{\text {macro }} / 12 \tau\right], \quad c_{L, \text { eff }}^{\text {macro }} \equiv c_{L}^{\text {bulk }}+c_{L, \text { eff }}^{\text {exterior }},
$$

and hence, for large $p$,

$$
B_{2 k}(\vec{Q}, p) \sim \exp \left[2 \pi \sqrt{c_{L, e f f}^{\text {macro }} p / 6}\right] .
$$

This is our general expression for the index $B_{2 k}$ for four dimensional black holes computed in the macroscopic theory. We shall describe the computation of $c_{L}^{\text {bulk }}$ and $c_{L, e f f}^{\text {exterior }}$ in sections 3 and 4 respectively. We shall in fact see that $c_{L, e f f}^{\text {macro }}$ is simpler to calculate than the individual contributions from the bulk and the exterior since the former is directly related to the coefficients of certain Chern-Simons terms in the effective action in the asymptotic space-time in which the black hole is embedded.

Let us now consider five dimensional black holes. The analysis goes through more or less in the same manner with $h$ replaced by $J_{R}$ provided that all the $\mathrm{SU}(2)_{L}$ singlet supersymmetry generators which are broken by the black hole solution are also broken by the $A d S_{3}$. The main difference arises from the fact that the exterior modes of the five dimensional black holes carry both $J_{L}$ and $J_{R}$ quantum numbers besides the momentum along $S^{1}$. Since we are summing over $J_{R}$ but keeping $J_{L}$ and the momentum along $S^{1}$ fixed in defining the index, the analog of (2.11) now takes the form

$$
C_{2 k}(\vec{q})=\sum_{\vec{q}_{\text {bulk }}} C_{\text {bulk }}\left(\vec{q}_{\text {bulk }}\right) C_{2 k, \text { exterior }}\left(\vec{q}-\vec{q}_{b u l k}\right),
$$

where the charge vector $\vec{q}$ now also includes the $J_{L}$ quantum number, and $C_{b u l k}$ denotes the trace of $e^{2 \pi i J_{R}}$. We now separate out two charges from the set $\vec{q},-$ the momentum $p$ along $S^{1}$ and the $\mathrm{U}(1)_{L} \subset \mathrm{SU}(2)_{L}$ charge $J_{L}=J / 2$ - and call the rest of the charges $\vec{Q}$. Denoting by $\sim$ the Fourier transforms in the charges $p$ and $J$, by $\tau$ and $z$ the variables conjugate to $p$ and $J$, and assuming that the exterior modes only carry $p$ and $J$ quantum numbers, we can express (2.19) as

$$
\widetilde{C}_{2 k}(\vec{Q}, \tau, z)=\widetilde{C}_{b u l k}(\vec{Q}, \tau, z) \widetilde{C}_{2 k ; \text { exterior }}(\tau, z) .
$$

\footnotetext{
${ }^{8}$ We should emphasize here that while the modularity of $\widetilde{B}_{2 k ; b u l k}(\vec{Q}, \tau)$ follows from the fact that in the $C F T_{2}$ dual to the $A d S_{3}$ the action of $h_{\text {bulk }}$ is chiral, the function $\widetilde{B}_{2 k \text {;exterior }}(\vec{Q}, \tau)$ is not a priori a modular form since the action of $h_{\text {bulk }}$ on the exterior modes is not chiral. Hence, to derive this asymptotics it is necessary to examine the behavior of $\widetilde{B}_{2 k \text {;exterior }}(\tau)$ explicitly as we describe in section 4 .
} 
In order to find the behaviour of $C_{2 k}$ in the Cardy limit we need to find the behaviour of $\widetilde{C}_{2 k}$ for small $\tau$. The behaviour of $\widetilde{C}_{b u l k}(\vec{Q}, \tau, z)$ for small $\tau$ can be found as follows. First we note that in $\mathrm{CFT}_{2}$ dual to the bulk of $A d S_{3}$ the $\mathrm{SU}(2)_{L}$ and $\mathrm{SU}(2)_{R}$ spatial rotations can be identified as the left- and right-moving SU(2) R-symmetry currents. From this it follows that if instead of $C_{b u l k}$ we had considered the degeneracy $d_{b u l k}$ of the left-moving excitations then for large $p$ and $J_{\sim}^{<} \sqrt{p}, d_{b u l k}(\vec{Q}, p, J)$ grows as $\exp \left[2 \pi \sqrt{c_{L}^{\text {bulk }}\left(p-\frac{J^{2}}{4 k_{L}^{\text {bulk }}}\right)}\right]$. Equivalently for small $\tau$ and $z_{\sim}^{<} 1$ we have

$$
\widetilde{d}_{b u l k}(\vec{Q}, \tau, z) \equiv \sum_{p, J} d_{b u l k}(\vec{Q}, p, J) e^{2 \pi i p \tau+2 \pi i J z} \sim \exp \left[\frac{\pi i c_{L}^{b u l k}}{12 \tau}-2 \pi i \frac{k_{L}^{b u l k} z^{2}}{\tau}\right]
$$

(2.21) is a consequence of the modular symmetry of the $\mathrm{CFT}_{2}$, and the exponent $-\frac{c_{L}^{\text {bulk }}}{12}+$ $k_{L}^{b u l k} z^{2}$ has the interpretation of the ground state energy of the left-moving sector of the CFT with the boundary condition twisted by $e^{2 \pi i J z}$ under $\sigma \rightarrow \sigma+2 \pi$. Now following the same logic as in the case of $\widetilde{B}_{b u l k}$ we can argue that for small $\tau, \widetilde{C}_{b u l k}$ will have the same behaviour as $\widetilde{d}_{b u l k}$, since under modular transformation the extra insertion of $(-1)^{2 J_{R}}$ in the trace will mapped to a twist by $(-1)^{2 J_{R}}$, and this, being a twist by the zero mode of a right-moving current, should not affect the ground state energy of the left-moving sector. Thus we get

$$
\widetilde{C}_{b u l k}(\vec{Q}, \tau, z) \sim \exp \left[\frac{\pi i c_{L}^{b u l k}}{12 \tau}-2 \pi i \frac{k_{L}^{b u l k} z^{2}}{\tau}\right]
$$

Furthermore we shall find in section 4 that for small $\tau$ and $z_{\sim}^{<} 1, \widetilde{C}_{2 k \text {;exterior }}(\tau, z)$ is given by a similar formula

$$
\widetilde{C}_{2 k ; \text { exterior }}(\tau, z) \sim \exp \left[\frac{\pi i c_{L, \text { eff }}^{\text {exterior }}}{12 \tau}-2 \pi i \frac{k_{L, \text { eff } f}^{\text {exterior }} z^{2}}{\tau}\right]
$$

eq.(2.20) now gives

$$
\begin{aligned}
& \widetilde{C}_{2 k}(\vec{Q}, \tau, z) \sim \exp \left[\frac{\pi i c_{L, e f f}^{\text {macro }}}{12 \tau}-2 \pi i \frac{k_{L, e f f}^{\text {macro }} z^{2}}{\tau}\right], \\
& c_{L, \text { eff }}^{\text {macro }} \equiv c_{L}^{\text {bulk }}+c_{L, \text { eff }}^{\text {exterior }}, \quad k_{L, \text { eff }}^{\text {macro }} \equiv k_{L}^{\text {bulk }}+k_{L, \text { eff }}^{\text {exterior }},
\end{aligned}
$$

and hence

$$
C_{2 k}(\vec{Q}, p, J) \sim \exp \left[2 \pi \sqrt{c_{L, e f f}^{\text {macro }}\left(p-\frac{J^{2}}{4 k_{L, e f f}^{\text {macro }}}\right)}\right] .
$$

Again we shall find that $c_{L, e f f}^{\text {macro }}$ and $k_{L, e f f}^{\text {macro }}$ are given in terms of the coefficients of certain Chern-Simons terms in the effective action in the asymptotic space-time, and hence are easier to calculate than the individual contributions from bulk and the exterior modes. 


\section{Macroscopic results for four and five dimensional black holes}

In this section we examine the macroscopic formulæ for the entropy of a certain class of four and five dimensional black holes in appropriate limits. Much work has been devoted to the study of corrections to black hole entropy due to a specific class of higher derivative terms obtained by supersymmetrizing the curvature squared terms, both in four and five dimensions $[6,10,11,39,70,71,81-83]$. However in this approach there is no a priori justification of including only a specific subset of higher derivative corrections to the effective action for computing the entropy. Our approach will be based on the method advocated in [60] where in certain limits the higher derivative corrections to the black hole entropy can be related to the coefficients of certain Chern-Simons terms in the effective action. Since these coefficients are integers, possible corrections to them are severely limited, and hence can often be computed. This will allow us to compute the black hole entropy in appropriate limits after including the effect of all possible higher derivative corrections.

In all subsequent discussions we shall use units in which $\alpha^{\prime}=1$, normalize the ten dimensional Einstein-Hilbert + dilaton action so that it takes the form

$$
(2 \pi)^{-7} \int d^{10} x \sqrt{-\operatorname{det} G} e^{-2 \Phi}\left[R+4(\nabla \Phi)^{2}\right]
$$

and normalize the $p$-form field strength so that its kinetic term has the form

$$
-\frac{1}{2} \frac{1}{p !}(2 \pi)^{-7} \int d^{10} x \sqrt{-\operatorname{det} G} e^{\kappa \Phi} F_{M_{1} \cdots M_{p}} F^{M_{1} \cdots M_{p}}
$$

for some appropriate constant $\kappa$.

\section{$3.1 \quad$ D1-D5-p system in type IIB on $K 3 \times S^{1}$}

We consider a system of $Q_{5}$ D5-branes wrapped on $K 3 \times S^{1},\left(Q_{1}+Q_{5}\right)$ D1-branes wrapped on $S^{1}$ and $n$ units of momentum along $S^{1}$. We choose the convention in which positive $n$ denotes left-moving momentum along $S^{1}$ and take $n$ to be positive. Since a D5-brane wrapped on K3 carries -1 unit of D1-brane charge, $Q_{1}$ represents the physical D1-brane charge carried by this system. Besides these charges we also make the system carry angular momentum. In five dimensions the spatial rotation group is $\mathrm{SU}(2)_{L} \times \mathrm{SU}(2)_{R}$. We shall consider D1-D5-p system of the type described above carrying $\mathrm{U}(1)_{L} \subset \mathrm{SU}(2)_{L}$ charge $J_{L}=J / 2$. Supersymmetry then forces the corresponding black hole solution to be invariant under $\mathrm{SU}(2)_{R}$, i.e. carry zero $\mathrm{SU}(2)_{R}$ charge. The entropy of a supersymmetric black hole carrying these charges, calculated using the two derivative action of the supergravity theory and the classical Bekenstein-Hawking formula, is given by [75]

$$
2 \pi \sqrt{Q_{1} Q_{5} n-\frac{J^{2}}{4}}
$$

Our goal will be to understand corrections to this formula in two different limits:

1. Type IIB Cardy limit: $n \rightarrow \infty$ with $Q_{1}, Q_{5}$ fixed. $|J|$ must be bounded by a term of order $\sqrt{n}$ so that $Q_{1} Q_{5} n-\frac{J^{2}}{4}$ scales as $n$. 
2. Type IIA Cardy limit: $Q_{1} \rightarrow \infty$ with $Q_{5}, n$ fixed. $|J|$ must be bounded by a term of order $\sqrt{Q_{1}}$ so that $Q_{1} Q_{5} n-\frac{J^{2}}{4}$ scales as $Q_{1}$.

The type IIB Cardy limit clearly corresponds to taking the momentum along the circle $S^{1}$ to infinity keeping other charges fixed in a type IIB frame. As we shall see, the type IIA Cardy limit corresponds to taking the momentum along the dual circle to infinity keeping the other charges fixed in a dual type IIA frame.

\subsubsection{Type IIB Cardy limit}

We begin by writing down the near horizon geometry of the black hole [75, 84] in the normalization convention of $[51,85]$ for the action and the solution:

$$
\begin{aligned}
d S^{2}= & r_{0} \frac{d \rho^{2}}{\rho^{2}}+d y^{2}+r_{0}\left(d x^{4}+\cos \theta d \phi\right)^{2}+\frac{J \lambda^{2}}{8 r_{0} R V} d y\left(d x^{4}+\cos \theta d \phi\right)-2 \sqrt{r_{0}} \rho d y d \tau \\
& +r_{0}\left(d \theta^{2}+\sin ^{2} \theta d \phi^{2}\right)+\widehat{g}_{m n} d u^{m} d u^{n}, \quad y \equiv y+2 \pi R \\
e^{\Phi}= & \lambda, \\
F^{(3)}= & \frac{r_{0}}{\lambda}\left[\epsilon_{3}+* \epsilon_{3}+\frac{J \lambda^{2}}{16 r_{0}^{2} R V} d y \wedge\left(\frac{1}{\rho} d \rho \wedge\left(d x^{4}+\cos \theta d \phi\right)+\sin \theta d \theta \wedge d \phi\right)\right],
\end{aligned}
$$

where $d S^{2}$ denotes the string metric, $\Phi$ denotes the dilaton, $F^{(3)}$ is the RR 3-form field strength, $g_{m n}$ is the metric on K3 with volume $(2 \pi)^{4} V, u^{m}$ 's are the coordinates on $K 3$, $\left(x^{4}, \theta, \phi\right)$ are the coordinates labelling a 3 -sphere $S^{3}, \epsilon_{3} \equiv \sin \theta d x^{4} \wedge d \theta \wedge d \phi$ is the volume form on this 3 -sphere satisfying $\int_{S^{3}} \epsilon_{3}=16 \pi^{2}$ and $* \epsilon_{3}$ denotes the Hodge-dual of $\epsilon_{3}$ in six dimensions. The attractor equations determine the near horizon parameters in terms of the charges via the relations

$$
r_{0}=\frac{\lambda Q_{5}}{4}, \quad V=\frac{Q_{1}}{Q_{5}}, \quad R=\sqrt{\frac{\lambda n}{Q_{1}}} .
$$

Note that $\lambda$, labeling the string coupling, is undetermined on the horizon. If $Q_{1}, Q_{5}, n$ are large but finite then by adjusting $\lambda$ we can keep the string coupling small, and the parameter $r_{0}$, that controls the length scale of the near horizon geometry, large. Thus in this case we have a systematic expansion in $\alpha^{\prime}$ and the string coupling, with the leading term in the expansion given by the Bekenstein-Hawking entropy. We shall try to go beyond this by taking only one of the charges to be large, keeping the other charges finite.

By a coordinate change

$$
\begin{aligned}
x^{4} & =\tilde{x}^{4}-\frac{J \lambda^{2}}{16 r_{0}^{2} R V} y, \quad y=\tilde{y}\left(1-\frac{J^{2} \lambda^{4}}{256 r_{0}^{3} R^{2} V^{2}}\right)^{-1 / 2}=\tilde{y}\left(1-\frac{J^{2}}{4 Q_{1} Q_{5} n}\right)^{-1 / 2}, \\
\tau & =\widetilde{\tau}\left(1-\frac{J^{2}}{4 Q_{1} Q_{5} n}\right)^{1 / 2},
\end{aligned}
$$

we can bring the metric to the form

$$
\begin{aligned}
d S^{2}= & r_{0}\left(\frac{d \rho^{2}}{\rho^{2}}-\rho^{2} d \tilde{\tau}^{2}\right)+\left(d \tilde{y}-\sqrt{r_{0}} \rho d \tilde{\tau}\right)^{2}+\widehat{g}_{m n} d u^{m} d u^{n} \\
& +r_{0}\left(\left(d \tilde{x}^{4}+\cos \theta d \phi\right)^{2}+d \theta^{2}+\sin ^{2} \theta d \phi^{2}\right)
\end{aligned}
$$


Except for the global identification implicit in the periods of the coordinates $\left(x^{4}, \theta, \phi, \tilde{y}\right)$ this metric has no dependence on $J$. In fact it has locally an $A d S_{3} \times S^{3}$ factor, with the coordinates $(\rho, \tau, y)$ labelling $A d S_{3}$ and $\left(\theta, \phi, x^{4}\right)$ labelling $S^{3}$ [86]. The appearance of the $A d S_{3} \times S^{3}$ factor allows us to apply the general reasoning given in [60] which we shall now review.

We begin with the observation that the classical Wald entropy given in (3.3) can be written in the form $[60,62,63,87]$

$$
S_{B H}=2 \pi \sqrt{\frac{c_{L}^{\text {bulk }}}{6}\left(n-\frac{J^{2}}{4 k_{L}^{\text {bulk }}}\right)},
$$

where

$$
c_{L}^{\text {bulk }}=6 Q_{1} Q_{5}, \quad k_{L}^{\text {bulk }}=Q_{1} Q_{5} .
$$

A physical explanation of this formula may be given as follows. If we take the limit in which the asymptotic radius $R_{a s}$ of the circle $S^{1}$ goes to infinity keeping fixed all the quantized charges and adjusting the other moduli so that the asymptotic geometry approaches a finite six dimensional background, then the black hole solution develops an intermediate region which contains an $A d S_{3} \times S^{3}$ factor and the near horizon configuration given in (3.4) appears as the near horizon geometry of an extremal BTZ black hole sitting inside the $A d S_{3}$ [61]. ${ }^{9}$ Furthermore this black hole carries a $\mathrm{U}(1)_{L}$ charge $J / 2$, with the $\mathrm{U}(1)_{L}$ interpreted as the abelian subgroup of the $\mathrm{SU}(2)_{L} \subset \mathrm{SU}(2)_{L} \times \mathrm{SU}(2)_{R}$ gauge group arising out of dimensional reduction on $S^{3}$. By AdS/CFT correspondence the states represented by this charged extremal BTZ black hole in this asymptotically $A d S_{3}$ geometry can now be regarded as RR sector states with $\left(\bar{L}_{0}=0, L_{0}=n\right)$ in the holographically dual $C F T_{2}$. Furthermore in $\mathrm{CFT}_{2}$ the $\mathrm{SU}(2)_{L} \times \mathrm{SU}(2)_{R}$ rotational symmetry of $S^{3}$ appears as the zero mode subalgebra of an $\mathrm{SU}(2)_{L} \times \mathrm{SU}(2)_{R}$ current algebra, with $\mathrm{SU}(2)_{L}$ being a left-moving current algebra and $\mathrm{SU}(2)_{R}$ a right-moving current algebra. Thus $J / 2$ represents the charge carried by the global part of the $\mathrm{U}(1)_{L} \subset \mathrm{SU}(2)_{L}$ current algebra. Eq. (3.8) can now be interpreted as the Cardy formula for the growth of states in the two dimensional conformal field theory, with $c_{L}^{b u l k}$ representing the central charge carried by the left moving component of the stress tensor of the $\mathrm{CFT}_{2}$, and $k_{L}^{b u l k}$ representing the level of the $\mathrm{SU}(2)_{L}$ current algebra.

In order to check that this interpretation is correct we must independently compute $c_{L}^{b u l k}$ and $k_{L}^{b u l k}$ from first principles and check that the result agrees with (3.9) computed from black hole entropy. For this it is also useful to introduce the quantities $c_{R}^{b u l k}$ which represents the central charge carried by the right moving component of the stress tensor of

\footnotetext{
${ }^{9}$ The asymptotic boundary of this $A d S_{3}$ space is the $(1+1)$ dimensional space labelled by $y$ and $\tau$, and the symmetry of the intermediate $A d S_{3} \times S^{3}$ includes the Lorentz transformation in this (1+1) dimensional space as well as the full rotation group of $S^{3}$. This may appear surprising since the black hole carries $-n$ units of momentum along $S^{1}$ which breaks Lorentz symmetry in the $y-\tau$ plane and angular momentum $J_{L}=J / 2$ which breaks the $\mathrm{SO}(4)$ rotational symmetry of $S^{3}$ to its $\mathrm{SU}(2)_{R}$ subgroup. The reason that this is not inconsistent is that if we take $R_{a s}$ to infinity keeping $n$ and $J$ fixed then the physical momentum $n / R_{a s}$ and the angular momentum per unit length $J / R_{a s}$ both vanish. Since these are the parameters which enter directly the black hole solution, it is not surprising that from the point of view of an asymptotic observer we recover the Lorentz invariance in the $\tau-y$ plane as well as the $\mathrm{SO}(4)$ rotational invariance in this limit.
} 
this $\mathrm{CFT}_{2}$ and $k_{R}^{\text {bulk }}$ that gives the level of the right-moving $\mathrm{SU}(2)_{R}$ current algebra. In the classical limit $c_{L}^{\text {bulk }}-c_{R}^{\text {bulk }}$ is given by the coefficient $c_{\text {grav }}^{\text {bulk }}$ of the Lorentz Chern-Simons term in the bulk theory, and $k_{L}^{b u l k}$ and $k_{R}^{b u l k}$ are given by the coefficients of the Chern-Simons terms involving $\mathrm{SU}(2)_{L}$ and $\mathrm{SU}(2)_{R}$ gauge fields in the bulk theory. Furthermore using the supersymmetry of the bulk theory one finds that the boundary $\mathrm{CFT}_{2}$ possesses $(0,4)$ superconformal symmetry. ${ }^{10}$ Thus the $\mathrm{SU}(2)_{R}$ current algebra can be identified as the R-symmetry algebra of the $(0,4)$ superconformal algebra, leading to the relation $c_{R}^{b u l k}=$ $6 k_{R}^{b u l k}[60]$. This gives:

$$
c_{L}^{\text {bulk }}-c_{R}^{\text {bulk }}=c_{\text {grav }}^{\text {bulk }}, \quad c_{R}^{\text {bulk }}=6 k_{R}^{\text {bulk }} .
$$

This allows us to express $c_{L}^{b u l k}$ as

$$
c_{L}^{b u l k}=c_{\text {grav }}^{\text {bulk }}+6 k_{R}^{b u l k} .
$$

In the specific example under consideration, there is no Lorentz Chern-Simons term in the supergravity approximation. Thus we have $c_{\text {grav }}^{\text {bulk }}=0$ and so $c_{R}^{\text {bulk }}=6 k_{R}^{\text {bulk }}$. Eq. (3.9) would then follow if we have $k_{L}^{\text {bulk }}=k_{R}^{\text {bulk }}=Q_{1} Q_{5}$. The proof of this, given in [88] has been reviewed in appendix A where we also give a generalization of this result.

So far we have just reinterpreted the classical Bekenstein-Hawking formula. But now we can turn the argument around to give a definition of the black hole entropy in the full quantum theory in the type IIB Cardy limit defined earlier. The main ingredient is the observation that for states carrying large $L_{0}$ the Cardy formula is valid in the $\mathrm{CFT}_{2}$ even in the quantum theory. Thus we can use (3.8) to compute the full quantum entropy associated with the bulk of $A d S_{3}$ in the large $n$ limit, provided $c_{L}^{\text {bulk }}$ represents the left-moving central charge in the full quantum theory, and $k_{L}^{b u l k}$ is the level of the $\mathrm{SU}(2)_{L}$ current algebra in the full quantum theory. ${ }^{11}$ Furthermore (3.11) will also continue to hold in the full quantum theory. Thus the problem reduces to the computation of $k_{L}^{b u l k}, k_{R}^{b u l k}$ and $c_{\text {grav }}^{\text {bulk }}$. As argued in section 2 these quantities also determine the contribution to the index from the modes living in the bulk of $A d S_{3}$. We still need to compute separately the contribution from the exterior modes to which we shall come back later.

Let us now discuss the computation of these quantites after taking into account higher derivative and quantum corrections. Since the coefficients of the Chern-Simons terms are quantized, $c_{\text {grav }}^{\text {bulk }}, k_{R}^{b u l k}$ and $k_{L}^{\text {bulk }}$ are quantized. It then follows from (3.11) that $c_{L}^{\text {bulk }}$ is also quantized. Thus these coefficients must be polynomial in the charges $Q_{1}, Q_{5}$ and cannot, for example, carry any inverse powers in the charges. This severely restricts the form of the corrections. Furthermore, we can use a generalization of the scaling argument of [74] to determine in which order in perturbation theory a given correction could arise. If we

\footnotetext{
${ }^{10}$ In fact in this particular example the $\mathrm{CFT}_{2}$ has $(4,4)$ superconformal supersymmetry and this allows us to relate $c_{L}^{\text {bulk }}$ directly to the coefficient $k_{L}^{\text {bulk }}$ of the $\mathrm{SU}(2)_{L}$ Chern-Simons terms in the bulk action via $c_{L}^{b u l k}=6 k_{L}^{b u l k}$. However in order to maintain a uniform discussion of all the cases we shall only make use of the $(0,4)$ supersymmetry of the $\mathrm{CFT}_{2}$.

${ }^{11}$ If we assume that the effect of quantum corrections can be encoded in a local 1PI action in $A d S_{3}$, then (3.8) can be derived directly in the bulk theory, either via euclidean action formalism [60] or via Wald's formula [63].
} 
take an extremal black hole carrying NS-NS sector electric charges $\bar{q}_{N S N S}^{(e l)}$, NS-NS sector magnetic charges $\vec{q}_{N S N S}^{(m a g)}$, and RR sector charges $\vec{q}_{R R}$, then the argument of [74] implies that the $l$-loop contribution to any of the coefficients $c_{\text {grav }}^{b u l k}, k_{R}^{b u l k}$ and $k_{L}^{b u l k}$ - collectively denoted by by $c^{(l)}$ - satisfies the scaling law:

$$
c^{(l)}\left(\vec{q}_{N S N S}^{(m a g)}, \lambda^{2} \vec{q}_{N S N S}^{(e l)}, \lambda \vec{q}_{R R}\right)=\lambda^{2-2 l} c^{(l)}\left(\vec{q}_{N S N S}^{(m a g)}, \vec{q}_{N S N S}^{(e l)}, \vec{q}_{R R}\right) .
$$

In our example, $Q_{1}, Q_{5}$ are $\mathrm{RR}$ sector charges. Thus the scaling relation takes the form

$$
c^{(l)}\left(\lambda Q_{1}, \lambda Q_{5}\right)=\lambda^{2-2 l} c^{(l)}\left(Q_{1}, Q_{5}\right) .
$$

Clearly the leading contribution to $k_{L}^{b u l k}$ and $k_{R}^{b u l k}$, given by $Q_{1} Q_{5}$, satisfies (3.13) with $l=0$, showing that this arises at the tree level. A correction to any of the coefficients $c_{\text {grav }}^{\text {bulk }}$, $k_{R}^{b u l k}$ and $k_{L}^{b u l k}$ linear in $Q_{1}$ or $Q_{5}$ will be suppressed with respect to the leading term by a power of $1 / \lambda$ under the scaling given in (3.13). According to (3.13) this must arise at $l=1 / 2$, i.e. at the 'half loop' order. Since close string perturbation theory includes only contributions from integral number of loops we see that we cannot get corrections to the central charge which are linear in $Q_{1}$ or $Q_{5}$. Put another way, a correction that is suppressed by a single power of $\mathrm{RR}$ charges must come from terms in the action involving odd number of RR fields. Such terms are not present in type IIB string theory. By following the same line of argument we see that a constant term in the central charge will produce an effect at the one loop order. Thus we might ask whether one loop correction in type IIB string theory could produce corrections to the Lorentz, $\mathrm{SU}(2)_{L}$ or $\mathrm{SU}(2)_{R}$ Chern-Simons term in the theory living on $A d S_{3}$. We can consider two possibilities. The first possibility is that such a term could arise from a one loop correction to the ten (or six) dimensional effective action integrated over $K 3 \times S^{3}$ (or $S^{3}$ ). Since the term we are looking for is independent of $Q_{1}$ and $Q_{5}$, it cannot involve the 3 -form fluxes and must be purely gravitational in nature. Now in an even dimensional theory it is impossible to write down a purely gravitational Chern-Simons term. Thus we do not get a constant contribution to $c_{L}^{\text {bulk }}$ by integrating a higher dimensional Chern-Simons term on $S^{3}$. The second possibility is that there can be one loop contributions to the Lorentz and/or $\mathrm{SU}(2)_{R}$ Chern-Simons terms which arise in the theory after compactification on $K 3 \times S^{3}$ and cannot be seen in the ten or six dimensional type IIB string theory. A priori we cannot rule out such a possibility; so let us denote such one loop contributions to $c_{\text {grav }}^{\text {bulk }}, k_{L}^{b u l k}$ and $k_{R}^{b u l k}$ by $A, B$ and $C$ respectively. This gives

$$
c_{L}^{b u l k}=6 Q_{1} Q_{5}+A+6 C, \quad k_{L}^{b u l k}=Q_{1} Q_{5}+B .
$$

$c_{L}^{b u l k}$ and $k_{L}^{\text {bulk }}$ given in (3.14) control the contribution to the black hole degeneracy/index from the bulk of $A d S_{3}$. To determine the full contribution to the macroscopic index using (2.24), (2.25) we must combine this with the contribution from the exterior degrees of freedom mentioned in the previous section. We shall show in section 4 that the exterior contributions $c_{L, e f f}^{\text {exterior }}$ and $k_{L, e f f}^{\text {exterior }}$ to the index precisely cancel the constant shifts $(A+6 C)$ and $B$ in eq.(3.14), leading to:

$$
c_{L, e f f}^{\text {macro }}=6 Q_{1} Q_{5}, \quad k_{L, e f f}^{\text {macro }}=Q_{1} Q_{5} .
$$


Using (2.25) we now see that the leading supergravity formula for the entropy is the complete contribution to the index in the Cardy limit:

$$
\ln d_{\text {macro }}\left(n, Q_{1}, Q_{5}, J\right) \simeq 2 \pi \sqrt{Q_{1} Q_{5} n-\frac{J^{2}}{4}} .
$$

Here $\simeq$ denotes equality up to corrections suppressed by inverse powers of $n$. The macroscopic result (3.16) agrees with the microscopic result (5.20) which will be derived in section 5 .

\subsubsection{Type IIA Cardy limit}

Let us turn to the type IIA Cardy limit: $Q_{1} \rightarrow \infty$ at fixed $n, Q_{5}$ and $J_{\sim}^{<} \sqrt{Q_{1}}$ [68]. The strategy will be to examine the black hole in a different duality frame in which $Q_{1}$ appears as a momentum along a circle, and then apply the same line of reasoning to find an exact formula for the black hole entropy in the limit $Q_{1} \rightarrow \infty$ at fixed $n, Q_{5}$. For this we first make an S-duality transformation in the ten dimensional type IIB string theory to map this system to an NS 5-brane, fundamental string, momentum system, and then make a T-duality along the circle $S^{1}$ to map this into a system in type IIA string theory on $K 3 \times \widetilde{S}^{1}$ with $Q_{5}$ NS 5-branes wrapped along $K 3 \times \widetilde{S}^{1}, n$ fundamental strings wrapped along $\widetilde{S}^{1}$ and $Q_{1}$ units of momentum along $\widetilde{S}^{1}$. By following the duality transformation rules and making a change of coordinates one finds that the near horizon geometry of the black hole in the type IIA variables, denoted by $\sim$, takes the form

$$
\begin{aligned}
d S^{2}= & \widetilde{r}_{0} \frac{d \rho^{2}}{\rho^{2}}+d y^{2}+\widetilde{r}_{0}\left(d x^{4}+\cos \theta d \phi\right)^{2}+\frac{J \widetilde{\lambda}^{2}}{8 \widetilde{r}_{0} \widetilde{R} \widetilde{V}} d y\left(d x^{4}+\cos \theta d \phi\right)-2 \sqrt{\widetilde{r}_{0}} \rho d y d \tau \\
& +\widetilde{r}_{0}\left(d \theta^{2}+\sin ^{2} \theta d \phi^{2}\right)+\widehat{g}_{m n} d u^{m} d u^{n}, \quad y \equiv y+2 \pi \widetilde{R} \\
e^{\Phi}= & \widetilde{\lambda} \\
\widetilde{H}^{(3)}= & \widetilde{r}_{0}\left[\epsilon_{3}+* \epsilon_{3}+\frac{J \widetilde{\lambda}^{2}}{16 \widetilde{r}_{0}^{2} \widetilde{R} \widetilde{V}} d y \wedge\left(\frac{1}{\rho} d \rho \wedge\left(d x^{4}+\cos \theta d \phi\right)+\sin \theta d \theta \wedge d \phi\right)\right],
\end{aligned}
$$

where $\widetilde{H}^{(3)}$ is the NS-NS 3-form field strength. The near horizon parameters are now given in terms of the charges and the parameter $\widetilde{\lambda}$ via the relations

$$
\widetilde{r}_{0}=\frac{Q_{5}}{4}, \quad \widetilde{V}=\widetilde{\lambda}^{2} \frac{n}{Q_{5}}, \quad \widetilde{R}=\sqrt{\frac{Q_{1}}{n}} .
$$

With the help of the same coordinate transformation (3.6) we can remove the explicit $J$ dependence of the solution except for in the periodic identification of the new coordinates. The space-time spanned by the coordinates $\left(\rho, \tau, y, \theta, \phi, x^{4}\right)$ is now locally $A d S_{3} \times S^{3}$. If we take the limit in which the asymptotic radius $\widetilde{R}_{a s}$ of $\widetilde{S}^{1}$ goes to infinity keeping fixed the quantized charges and the six dimensional background, then the solution develops an $A d S_{3} \times S^{3}$ factor in the intermediate region, and the near horizon geometry described in (3.17) can be regarded as that of an extremal charged BTZ black hole embedded in this asymptotically $A d S_{3} \times S^{3}$ geometry. In the holographically dual $\mathrm{CFT}_{2}$ the BTZ black hole 
can now be regarded as an RR sector state with $L_{0}=Q_{1}, \bar{L}_{0}=0$ and $\mathrm{U}(1)_{L} \subset \mathrm{SU}(2)_{L}$ charge $J / 2$. Thus the entropy of the black hole in the limit of large $Q_{1}$ should be given by the Cardy formula

$$
S_{B H} \simeq 2 \pi \sqrt{\tilde{c}_{L}^{b u l k}\left(Q_{1}-\frac{1}{4}\left(\tilde{k}_{L}^{b u l k}\right)^{-1} J^{2}\right) / 6},
$$

where now $\tilde{c}_{L}^{b u l k}, \tilde{c}_{R}^{b u l k}, \tilde{k}_{L}^{\text {bulk }}$, and $\tilde{k}_{R}^{\text {bulk }}$ denote respectively the central charges of the left and right-moving Virasoro algebras and the levels of $\mathrm{SU}(2)_{L}$ and $\mathrm{SU}(2)_{R}$ current algebras in the $\mathrm{CFT}_{2}$. As before, $\tilde{c}_{\text {grav }}^{\text {bulk }} \equiv \tilde{c}_{L}^{b u l k}-\tilde{c}_{R}^{b u l k}$ is related to the coefficient of the Lorentz Chern-Simons term in the bulk and $\tilde{k}_{L}^{b u l k}$ and $\tilde{k}_{R}^{b u l k}$ are related to the coefficients of the $\mathrm{SU}(2)_{L}$ and $\mathrm{SU}(2)_{R}$ Chern-Simons terms. Furhermore using the supersymmetries of the bulk theory one can show that the $\mathrm{CFT}_{2}$ has $(0,4)$ supersymmetry. This leads to the relation $\tilde{c}_{R}^{b u l k}=6 \tilde{k}_{R}^{b u l k}$ and gives

$$
\tilde{c}_{L}^{b u l k}=\tilde{c}_{g r a v}^{b u l k}+6 \tilde{k}_{R}^{b u l k} .
$$

Comparison with (3.3) shows that in the supergravity approximation we have $\tilde{c}_{L}^{b u l k}=6 n Q_{5}$ and $\tilde{k}_{L}^{\text {bulk }}=n Q_{5}$. Since in this approximation there is no Lorentz Chern-Simons term in the action, $\tilde{c}_{\text {grav }}^{b u l k}$ vanishes and (3.20) gives $\tilde{k}_{R}^{b u l k}=n Q_{5}$. Direct computation of $\tilde{k}_{L}^{b u l k}$ and $\tilde{k}_{R}^{b u l k}$ can be performed using the procedure reviewed in appendix A and agrees with the values given above. Our goal now is to compute the corrections to $\tilde{c}_{\text {grav }}^{\text {bulk }}, \tilde{k}_{L}^{\text {bulk }}$ and $\tilde{k}_{R}^{\text {bulk }}$ due to higher derivative and string loop corrections.

Since $\tilde{c}_{g r a v}^{b u l k}, \tilde{k}_{L}^{b u l k}$ and $\tilde{k}_{R}^{b u l k}$ are all quantized, corrections to them could involve terms linear in $Q_{5}$ and/or $n$ and constant term. Now since $n$ represents an NSNS sector electric charge and $Q_{5}$ an NSNS sector magnetic charge, the scaling relation (3.12) takes the form

$$
\tilde{c}^{(l)}\left(\lambda^{2} n, Q_{5}\right)=\lambda^{2-2 l} \tilde{c}^{(l)}\left(n, Q_{5}\right),
$$

where $\tilde{c}^{(l)}$ stands for $l$ loop contribution to any of the quantities $\tilde{c}_{\text {grav }}^{b u l k}, \tilde{k}_{L}^{b u l k}$ and $\tilde{k}_{R}^{b u l k}$. This shows that a term linear in $n$, if present, must arise at string tree level. Since this term would be linear in $n$, representing the NS-NS 3-form flux $\widetilde{H}^{(3)}$ through $A d S_{3}$, it will have to arise from a six dimensional Chern-Simons term of the form $\int{ }^{D} \widetilde{H}^{(3)} \wedge \Omega_{C S}$ where $\Omega_{C S}$ is a Lorentz Chern-Simons 3-form in six dimensions, and ${ }^{D}$ denotes the dual field strength obtained by taking the Hodge dual of the flux $\delta S / \delta \widetilde{H}^{(3)}$ [89]. But tree level type IIA string theory does not have such a term in the action since the gauge invariant three form field strength in type II string theories do not involve a Lorentz Chern-Simons term. This shows that there are no corrections linear in $n$. According to the scaling relation (3.21) the constant term, if present, must arise at one loop. Since it does not involve any charges, it will have to either come from a purely gravitational term in ten dimensions which upon dimensional reduction on $K 3 \times S^{3}$ will produce a Lorentz Chern-Simons term in $A d S_{3}$, or arise as a one loop effect in the theory after compactification on $S^{3}$. Since there are no purely gravitational Chern-Simons terms in ten or six dimensions, we can rule out the first possibility. But as in the case of type IIB Cardy limit, we cannot rule out the second possibility. Let us denote such contributions to $\tilde{c}_{\text {grav }}^{b u l k}, \tilde{k}_{L}^{b u l k}$ and $\tilde{k}_{R}^{b u l k}$, if present, by $\widetilde{A}, \widetilde{B}$ and $\widetilde{C}$ respectively. 
Finally a term linear in $Q_{5}$, if present, must arise at one loop order, and come from a term proportional to $\int \widetilde{H}^{(3)} \wedge \Omega_{C S}$ in six dimensions. Are there such one loop corrections to the Chern-Simons term? The ten dimensional type IIA string theory indeed contains a one loop Chern-Simons term of the form

$$
-\frac{1}{2 \pi} \int \widetilde{B} \wedge I_{8}(X)
$$

where $\widetilde{B}$ is the NS-NS 2-form field and $I_{8}(X)=\frac{1}{48}\left(p_{2}(X)-\frac{p_{1}^{2}(X)}{4}\right), X$ being the ten dimensional space and $p_{n}$ denoting the $n$th Pontryagin class [90]. Upon dimensional reduction on $K 3$ this generates a term proportional to $\int \widetilde{H}^{(3)} \wedge \Omega_{C S}$. Thus $\tilde{c}_{\text {grav }}^{\text {bulk }}, \tilde{k}_{L}^{\text {bulk }}$ and $\tilde{k}_{L}^{b u l k}$ can all receive corrections linear in $Q_{5}$. To compute the coefficients of these terms we introduce the quantities $I_{7}^{0}$ and $p_{1}^{0}$ via the relations $I_{8}=d I_{7}^{0}$ and $p_{1}=d p_{1}^{0}$. Since $\widetilde{H}^{(3)}$ has nontrivial flux over $S^{3}$, the 2 -form field $\widetilde{B}$ is not well defined. Thus instead of taking the coupling (3.22) we shall take

$$
\frac{1}{2 \pi} \int \widetilde{H}^{(3)} \wedge I_{7}^{0}
$$

by integration by parts. Now the spin connection in the Kaluza-Klein reduction is simply a direct sum of the connections on $A d S_{3} \times S^{3} \times K 3$. Using the fact that the total pontryagin class of a direct sum satisfies $p(E \oplus F)=p(E) p(F)$, that $\int_{K 3} p_{1}=48$, and that $p_{1}=$ $-d \omega_{v}(\Gamma) / 8 \pi^{2}$ where

$$
\omega_{v}(\Gamma)=\operatorname{Tr}_{v}\left(\Gamma \wedge d \Gamma+\frac{2}{3} \Gamma \wedge \Gamma \wedge \Gamma\right)
$$

the trace being taken over the vector representation, we can express the contribution from $(3.23)$ as

$$
-\frac{1}{32 \pi^{3}} \int_{A d S_{3} \times S^{3}} H^{(3)} \wedge \omega_{v}(\Gamma)
$$

where $\Gamma$ now stands for the spin connection on $A d S_{3} \times S^{3}$. Using eqs.(A.14), (A.19) we see that the effect of (3.25) is to generate the following corrections to $\tilde{c}_{\text {grauv }}^{\text {grav }}, \tilde{k}_{R}^{\text {bulk }}$ and $\tilde{k}_{L}^{\text {bulk: }}$ :

$$
\Delta \tilde{c}_{\text {grav }}^{\text {bulk }}=12 Q_{5}, \quad \Delta \tilde{k}_{R}^{\text {bulk }}=Q_{5}, \quad \Delta \tilde{k}_{L}^{\text {bulk }}=-Q_{5} .
$$

We can check the consistency of the overall sign and normalization by setting $Q_{5}=1$; in this case the system is equivalent to a fundamental heterotic string which has $c_{\text {grav }}=12$. Combining (3.26) with the leading supergravity results and the constant shifts we arrive at the relations:

$$
\begin{aligned}
& \tilde{k}_{R}^{\text {bulk }}=Q_{5}(n+1)+\tilde{C}, \quad \tilde{k}_{L}^{b u l k}=Q_{5}(n-1)+\tilde{B}, \quad \tilde{c}_{\text {grav }}^{\text {bulk }}=12 Q_{5}+\tilde{A}, \\
& \tilde{c}_{L}^{b u l k}=\tilde{c}_{\text {grav }}^{\text {bulk }}+6 \tilde{k}_{R}^{\text {bulk }}=6 Q_{5}(n+3)+\tilde{A}+6 \tilde{C} .
\end{aligned}
$$

We now need to use (2.24), (2.25) to find the asymptotic formula for the index. Again we shall see in section 4 that the net effect of the exterior contribution $\tilde{c}_{L, \text { eff }}^{\text {exterior }}$ and $\tilde{k}_{L, \text { eff }}^{\text {exter }}$ is to cancel the terms proportional to $\widetilde{A}+6 \widetilde{C}$ and $\widetilde{B}$ in $\tilde{c}_{L}^{b u l k}$ and $\tilde{k}_{L}^{b u l k}$. Thus the growth 
of the macroscopic index $d_{\text {macro }}$ in the type IIA Cardy limit $Q_{1} \rightarrow \infty$ for fixed $Q_{5}, n$ will be controlled by the constants

$$
\tilde{k}_{L, e f f}^{\text {macro }}=Q_{5}(n-1), \quad \tilde{c}_{L, \text { eff }}^{\text {macro }}=6 Q_{5}(n+3),
$$

and $\ln d_{\text {macro }}$ given by

$$
\ln d_{\text {macro }}\left(n, Q_{1}, Q_{5}\right) \simeq 2 \pi \sqrt{Q_{5}(n+3)\left(Q_{1}-\frac{J^{2}}{4 Q_{5}(n-1)}\right)},
$$

where $\simeq$ implies equality up to corrections suppressed by powers of $Q_{1}$. This agrees with the result found in $[68,83]$ for small $J$ and large $n$ computed using a particular four derivative correction to the five dimensional effective action. Also the result for $\tilde{c}_{L, e f f}^{\text {macro }}$ agrees with the one computed in [91, 92] (see also [93, 94]) assuming a specific structure of all the higher derivative correction to the effective action. ${ }^{12}$ Most importantly (3.29) agrees with the microscopic answer (5.27) which will be derived in section 5 .

\subsection{Entropy of some four dimensional black holes}

We now consider a four dimensional theory obtained by compactifying type IIB string theory on $K 3 \times S^{1} \times \widetilde{S}^{1}$. In this theory we take the non-spinning D1-D5-p system analyzed in section 3.1 and place it in the background of $K$ Kaluza-Klein (KK) monopoles associated with the circle $\widetilde{S}^{1}$. Since for $K=1$ this system has the same near horizon geometry as the five dimensional D1-D5-p system analyzed in section 3.1, the macroscopic computation of the index is identical to that in section 3.1 except for the difference in the contribution due to the exterior modes. We shall however keep $K$ arbitrary and compute the entropy in a different duality frame in which we regard them as black holes in M-theory on $K 3 \times$ $T^{3}$ carrying M5-brane charges and internal momentum. For this we first make a mirror symmetry transformation in $K 3$ to take the D1-D5 system to a D3-D3 system with $Q_{1}$ D3-branes wrapped on $C_{2} \times S^{1}$ and $Q_{5}$ D3-branes wrapped on a $\widetilde{C}_{2} \times S^{1}$ where $C_{2}$ and $\widetilde{C}_{2}$ are a pair of dual 2-cycles of $K 3$. We then make a T-duality along the circle $\widetilde{S}^{1}$ to take the D3-branes to D4-branes and the KK monopoles to NS 5-branes wrapped on $K 3 \times S^{1}$. If we denote by $\widehat{S}^{1}$ the T-dual circle then we have $Q_{1}$ D4-branes along $C_{2} \times S^{1} \times \widehat{S}^{1}, Q_{5}$ D4-branes wrapped along $\widetilde{C}_{2} \times S^{1} \times \widehat{S}^{1}$, and $K$ NS 5-branes along $K 3 \times S^{1}$, carrying $n$ units of momentum along $S^{1}$. We can now regard the type IIA string theory as M-theory compactified on a new circle $S_{M}^{1}$, so that we have M-theory on $K 3 \times S^{1} \times \widehat{S}^{1} \times S_{M}^{1}$. The dyon configuration now corresponds to $Q_{1}$ M5-branes along $C_{2} \times S^{1} \times \widehat{S}^{1} \times S_{M}^{1}, Q_{5}$ M5-branes wrapped along $\widetilde{C}_{2} \times S^{1} \times \widehat{S}^{1} \times S_{M}^{1}$, and $K$ M5-branes wrapped along $K 3 \times S^{1}$, carrying $n$ units of momentum along $S^{1}$.

Our goal in this section will be to analyze the black hole solution corresponding to these charges and find the macroscopic entropy of this system in the limit $n \rightarrow \infty$, keeping the other charges fixed. Since the analysis proceeds more or less in the same way as for five dimensional black holes, our discussion will be brief. As in the case of the D1-D5-p system one finds that near the horizon the $A d S_{2} \times S^{2}$ appearing in the near horizon geometry

\footnotetext{
${ }^{12}$ Earlier results on this can be found in [95].
} 
of the black hole combines with the circle $S^{1}$ to produce a locally $A d S_{3} \times S^{2}$ factor [60]. Furthermore if we take the limit in which the asymptotic radius of $S^{1}$ approaches infinity, keeping fixed all other quantized charges and the five dimensional geometry in the M-theory frame then the M-theory background develops an intermediate $A d S_{3} \times S^{2}$ geometry, and the near horizon geometry of the black hole appears as the near horizon geometry of an extremal BTZ black hole embedded in this asymptotically $A d S_{3} \times S^{2}$ space. Thus applying the Cardy formula we see that the entropy is given by the formula

$$
S_{B H} \simeq 2 \pi \sqrt{c_{L}^{b u l k} n / 6},
$$

where $c_{L}^{b u l k}$ is the central charge of the left-moving Virasoro algebra of the holographically dual $\mathrm{CFT}_{2}$. In the supergravity approximation $c_{L}^{\text {bulk }}=6 Q_{1} Q_{5} K$, reproducing the Bekenstein-Hawking result $2 \pi \sqrt{Q_{1} Q_{5} K n}$ for the entropy [96, 97].

In the limit $n \rightarrow \infty$ with $Q_{1}, Q_{5}, K$ fixed, the complete contribution to the entropy (and the index) from the bulk modes on $A d S_{3}$ continues to be given by (3.30) provided $c_{L}^{\text {bulk }}$ represents the exact central charge of the left-moving Virasoro algebra after taking into account higher derivative and quantum corrections. As usual $\left(c_{L}^{\text {bulk }}-c_{R}^{\text {bulk }}\right)$ is given by the coefficient $c_{\text {grav }}^{\text {bulk }}$ of the Lorentz Chern-Simons term in $A d S_{3}$. On the other hand using the supersymmetries of the bulk geometry one can show that the dual $\mathrm{CFT}_{2}$ on the boundary has $(0,4)$ superconformal symmetry acting on the right-movers. As a result $c_{R}^{\text {bulk }}$ can be related to the level $k_{R}^{b u l k}$ of the $\mathrm{SU}(2)$ R-symmetry current in the $\mathrm{CFT}_{2}$ via the relation $c_{R}^{b u l k}=6 k_{R}^{b u l k}{ }^{13}$ Since this $\mathrm{SU}(2)$ current in the boundary theory is holographically dual to the $\mathrm{SU}(2)$ gauge fields in the bulk arising from dimensional reduction on $S^{2}, k_{R}^{b u l k}$ is given by the coefficient of the $\mathrm{SU}(2)$ Chern-Simons term in the bulk. This allows us to determine $c_{L}^{b u l k}$ in terms of the coefficients of the Chern-Simons terms in $A d S_{3}$ via the relations

$$
c_{L}^{\text {bulk }}=c_{\text {grav }}^{\text {bulk }}+6 k_{R}^{\text {bulk }} .
$$

The relevant Chern-Simons terms were evaluated in [60] for M-theory compactified on $M \times S^{1}$ where $M$ is a general Calabi-Yau 3-fold. In this theory, consider a black hole corresponding to M5-brane wrapped on $P \times S^{1}$ where $P$ is some general 4-cycle in $M$. Using the isomorphism between 4-cycles and 2-forms we can associate with $P$ a 2 -form on $M$ which we shall also denote by $P$. Then the result of [60] for $c_{L}^{b u l k}$ and $c_{R}^{b u l k}$ are:

$c_{R}^{\text {bulk }}=\int_{M}\left(P \wedge P \wedge P+\frac{1}{2} P \wedge c_{2}(M)\right)+\bar{A}_{R}, \quad c_{L}^{\text {bulk }}=\int_{M}\left(P \wedge P \wedge P+P \wedge c_{2}(M)\right)+\bar{A}_{L}$,

where $c_{2}(M)$ is the second Chern class of $M$. Note that we have allowed for constant shift $\left(\bar{A}_{L}, \bar{A}_{R}\right)$ in the central charges due to one loop effects arising after compactification of $M$-theory on $K 3 \times T^{2} \times S^{2} \times A d S_{3}$. Computation in [60] was carried out by integrating the quantum corrected ten dimensional Lagrangian density on $K 3 \times S^{3}$, and ignored possible quantum corrections which could arise after compactification on $K 3 \times S^{3}$. Evaluating this for the configuration we have, we get

$$
c_{R}^{\text {bulk }}=6 K\left(Q_{1} Q_{5}+2\right)+\bar{A}_{R}, \quad c_{L}^{\text {bulk }}=6 K\left(Q_{1} Q_{5}+4\right)+\bar{A}_{L} .
$$

\footnotetext{
${ }^{13}$ Although there is now a single $\mathrm{SU}(2)$ we shall label its anomaly coefficient by $k_{R}$.
} 
Again we shall see in section 4 that when we compute the full index in the macroscopic theory using (2.17), (2.18), the net effect of the exterior contribution $c_{L, e f f}^{\text {exterior }}$ is to cancel the $\bar{A}_{L}$ term in $c_{L}^{b u l k}$, giving rise to

$$
c_{L, \text { eff }}^{\text {macro }}=6 K\left(Q_{1} Q_{5}+4\right) .
$$

eq.(2.18) now shows that the index computed in the macroscopic theory grows as

$$
\ln d_{\text {macro }}\left(n, Q_{1}, Q_{5}, K\right) \simeq 2 \pi \sqrt{K\left(Q_{1} Q_{5}+4\right) n} \text { for large } n .
$$

This is in perfect agreement with the microscopic result (5.36) to be derived in section 5 .

\subsection{Black holes in toroidally compactified type IIB string theory}

In this subsection we shall repeat the analysis of the previous subsections for black holes in toroidally compactified type IIB string theory. Since the analysis proceeds in a more or less identical manner we shall mainly state the results without going through the details of the analysis.

First we consider the D1-D5-p system wrapped on $T^{4} \times S^{1}$. We shall use the same notation for the charges as in the case of $K 3 \times S^{1}$ compactification, except that now $Q_{1}$ represents the actual number of D1-branes since D5-branes wrapped on $T^{4}$ do not carry any D1-brane charge. In the limit when $Q_{1}, Q_{5}$ are fixed and $n$ becomes large, we get the result:

$$
\ln d_{\text {macro }}\left(n, Q_{1}, Q_{5}, J\right) \simeq \pi \sqrt{4 Q_{1} Q_{5} n-J^{2}} .
$$

In the limit of fixed $n, Q_{5}$ and $Q_{1}$ large, we have

$$
\ln d_{\text {macro }}\left(n, Q_{1}, Q_{5}, J\right) \simeq \pi \sqrt{4 Q_{1} Q_{5} n-J^{2}} .
$$

Derivation of (3.36) is a straightforward generalization of the similar analysis for type IIB on $K 3 \times S^{1}$ leading to (3.16). The main difference between the analysis leading to (3.37) and that leading to (3.29) is that the dimensional reduction of the $\int \widetilde{B} \wedge I_{8}$ term on $T^{4}$ does not produce any Chern-Simons term. Thus all corrections to $\tilde{c}_{L}^{b u l k}$ and $\tilde{k}_{L}^{b u l k}$ from the supergravity results, except for possible constant shifts from one loop corrections, vanish. The constant shift is cancelled by the contribution from the exterior modes due to the results of section 4. Using these results we arrive at (3.37). This is in perfect agreement with the microscopic result (5.43) to be derived in section 5 .

If we now consider a four dimensional black hole obtained by placing this system in the background of $K \mathrm{KK}$ monopoles, and go to the duality frame in which the system is described by momentum carrying M5-brane wrapped on $T^{7}$, then we can analyze the macroscopic entropy of the system following the same procedure as in section 3.2. In this case the near horizon geometry is locally $T^{6} \times A d S_{3} \times S^{2}$. The central charges $c_{L}^{\text {bulk }}$ and $c_{R}^{b u l k}$ associated with this $A d S_{3}$ are given by formulæ similar to those given in (3.33) except that now $\int P \wedge c_{2}(M)$ vanishes. Possible constant shift in $c_{L}^{b u l k}$ due to one loop correction is exactly cancelled by the hair contribution. This gives

$$
\ln d_{\text {macro }}\left(n, Q_{1}, Q_{5}, K\right) \simeq 2 \pi \sqrt{Q_{1} Q_{5} K n} \text { for large } n .
$$

This is in complete agreement with the macroscopic result (5.46). 


\section{Analysis of the exterior contribution}

In this section we shall compute the coefficients $c_{L, e f f}^{\text {exterior }}$ and $k_{L, e f f}^{\text {exterior }}$ appearing in (2.16) and (2.23) and show that their effect is to cancel the charge independent constant terms in the expressions for $c_{L, e f f}^{\text {macro }}$ and $k_{L, e f f}^{\text {macro }}$ which arise from one loop quantum corrections and which cannot be obtained as the dimensional reduction of the 1PI action in ten dimensions on the intermediate $A d S_{3}$ geometry. Examples of such terms are $A+6 C$ and $B$ in (3.14). We shall describe our analysis in the context of the five dimensional black hole, but it will be clear that the result we derive is also valid in four dimensions, the only difference being the absence of any reference to the $\mathrm{SU}(2)_{L}$ symmetry and the associated anomaly coefficient $k_{L}$ in four dimensions.

We begin by recollecting some relevant results from section 3. Recall that $c_{L}^{b u l k}$ is computed in section 3 via the relation

$$
c_{L}^{b u l k}=c_{\text {grav }}^{b u l k}+6 k_{R}^{b u l k},
$$

where $k_{R}^{\text {bulk }}$ and $c_{\text {grav }}^{\text {bulk }}$ are the coefficeints of the $\mathrm{SU}(2)_{R}$ and Lorentz Chern-Simons terms in the intermediate $A d S_{3}$ geomery. On the other hand $k_{L}^{\text {bulk }}$ was given by the coefficient of the $\mathrm{SU}(2)_{L}$ Chern-Simons term in the $A d S_{3}$ geometry. Part of the contribution to these ChernSimons terms came from integrating ten dimensional Chern-Simons terms on $K 3 \times S^{3}$, but this left open the possibility of constant one loop corrections to these coefficients which arise after compactification on $S^{3}$. Now imagine that instead of doing this reduction on the $K 3 \times S^{3}$ that arises in the intermediate $A d S_{3}$ region, we do this in the asymptotic region where the geometry is locally $K 3 \times R^{6} .{ }^{14}$ Let us take a thick spherical shell of large radius around the origin, bounded by the hypersurfaces $r=r_{1}$ and $r=r_{2}$ for large $r_{1}, r_{2}$, and regard this space as locally $R^{3} \times K 3 \times S^{3}$, with $S^{3}$ labelling the angular coordinates and $R^{3}$ containing the time coordinate, the radial coordinate $r$ and the coordinate along $S^{1}$. We can now formally dimensionally reduce the ten dimensional action on $K 3 \times S^{3}$ to calculate the coefficients of the Lorentz and $\mathrm{SU}(2)_{R} \times \mathrm{SU}(2)_{L}$ Chern-Simons terms on $R^{3}$. The calculation is identical to the one described in appendix $\mathrm{A}$ for the intermediate $A d S_{3}$ geometry, except that this time we do not expect any additional one loop correction due to compactification on $S^{3}$ since we are really doing the computation in $K 3 \times R^{6}$ rather than on $K 3 \times S^{3} \times A d S_{3}$. Thus the result for these coefficients will be identical to $c_{\text {grav }}^{\text {bulk }}$, $k_{R}^{b u l k}=c_{R}^{b u l k} / 6$ and $k_{L}^{b u l k}$ computed in section 3 and appendix A except for the constant one loop shifts. We shall denote these coefficients by $c_{g r a v}^{a s y m p}, k_{R}^{a s y m p}$ and $k_{L}^{a s y m p}$ respectively. For completeness we shall list below the values of $c_{\text {grav }}^{\text {asymp }}, k_{R}^{\text {asymp }}$ and $k_{L}^{\text {asymp }}$ for each of the systems analyzed in section 3 :

1. D1-D5-p system in type IIB on $K 3 \times S^{1}$ in the type IIB Cardy limit:

$$
c_{\text {grav }}^{\text {asymp }}=0, \quad k_{R}^{\text {asymp }}=Q_{1} Q_{5}, \quad k_{L}^{\text {asymp }}=Q_{1} Q_{5} .
$$

\footnotetext{
${ }^{14}$ Recall that we have taken the asymptotic radius of $S^{1}$ to infinity so that we have a $(5+1)$ dimensional asymptotic space-time.
} 
2. D1-D5-p system in type IIB on $K 3 \times S^{1}$ in the type IIA Cardy limit:

$$
c_{\text {grav }}^{\text {asymp }}=12 Q_{5}, \quad k_{R}^{a s y m p}=Q_{5}(n+1), \quad k_{L}^{a s y m p}=Q_{5}(n-1) .
$$

3. Four dimensional black hole in M-theory on $K 3 \times T^{2} \times S^{1}$ :

$$
c_{\text {grav }}^{\text {asymp }}=12 K, \quad k_{R}^{\text {asymp }}=K\left(Q_{1} Q_{5}+2\right) .
$$

4. D1-D5-p system in type IIB on $T^{4} \times S^{1}$ in the type IIB Cardy limit:

$$
c_{\text {grav }}^{\text {asymp }}=0, \quad k_{R}^{\text {asymp }}=Q_{1} Q_{5}, \quad k_{L}^{\text {asymp }}=Q_{1} Q_{5} .
$$

5. D1-D5-p system in type IIB on $T^{4} \times S^{1}$ in the type IIA Cardy limit:

$$
c_{\text {grav }}^{\text {asymp }}=0, \quad k_{R}^{\text {asymp }}=Q_{1} Q_{5}, \quad k_{R}^{\text {asymp }}=Q_{1} Q_{5} .
$$

6. Four dimensional black hole in M-theory on $T^{6} \times S^{1}$ :

$$
c_{\text {grav }}^{\text {asymp }}=0, \quad k_{R}^{a s y m p}=K Q_{1} Q_{5} .
$$

We shall now try to express the difference between the Chern-Simons coefficients calculated in the asymptotic geometry and the intermediate $A d S_{3}$ geometry in terms of some known quantities and in the process gain knowledge about the constant terms in the expression for the Chern-Simons coefficients in the intermediate $A d S_{3}$ region. For this we note that the coefficients of the Chern-Simons terms can also be interpreted as certain anomaly coefficients. For example $k_{R}^{b u l k}$ and $k_{L}^{b u l k}$ reflect the change in the effective action in the bulk theory by certain boundary terms in the intermediate $A d S_{3}$ geometry under $\mathrm{SU}(2)_{R}$ and $\mathrm{SU}(2)_{L}$ gauge transformations, and $c_{\text {grav }}^{\text {bulk }}$ reflects a similar change under local Lorentz transformations. $k_{R}^{a s y m p}, k_{L}^{a s y m p}$ and $c_{\text {grav }}^{\text {asymp }}$ reflect similar anomalies under local $\mathrm{SU}(2)_{R}$, $\mathrm{SU}(2)_{L}$ and Lorentz transformations in the asymptotic region. Thus the difference between $k_{R}^{\text {asymp }}$ and $k_{R}^{b u l k}$ must be accounted for by the contribution to the $\mathrm{SU}(2)_{R}$ anomaly due to the exterior degrees of freedom sitting between the asymptotic observer and the $A d S_{3}$. We shall denote this by $k_{R}^{\text {exterior }}$. An identical argument holds for $k_{L}$ and $c_{\text {grav }}$. Thus we have

$$
k_{R}^{\text {asymp }}=k_{R}^{\text {bulk }}+k_{R}^{\text {exterior }}, \quad k_{L}^{\text {asymp }}=k_{L}^{\text {bulk }}+k_{L}^{\text {exterior }}, \quad c_{\text {grav }}^{\text {asymp }}=c_{\text {grav }}^{\text {bulk }}+c_{\text {grav }}^{\text {exterior }} .
$$

Using (2.24), (4.1) and (4.8) we get

$$
\begin{aligned}
& c_{L, \text { eff }}^{\text {macro }}=c_{\text {grav }}^{\text {asymp }}-c_{\text {grav }}^{\text {exterior }}+6\left(k_{R}^{\text {asymp }}-k_{R}^{\text {exterior }}\right)+c_{L, \text { eff }}^{\text {exterior }}=c_{\text {grav }}^{\text {asymp }}+6 k_{R}^{\text {asymp }}+\Delta, \\
& k_{L, \text { eff }}^{\text {macro }}=k_{L}^{\text {asymp }}+\delta,
\end{aligned}
$$

where

$$
\begin{aligned}
\Delta \equiv-6 k_{R}^{\text {exterior }}-c_{\text {grav }}^{\text {exterior }}+c_{L, \text { eff }}^{\text {exterior }} & =-6 k_{R}^{\text {exterior }}-\left(c_{L}^{\text {exterior }}-c_{R}^{\text {exterior }}\right)+c_{L, e f f}^{\text {exterior }}, \\
\delta & =k_{L, \text { eff }}^{\text {exterior }}-k_{L}^{\text {exterior }} .
\end{aligned}
$$


Now we have already argued that the results for $c_{\text {grav }}^{\text {asymp }}, k_{R}^{\text {asymp }}$ and $k_{L}^{\text {asymp }}$ are identical to those of $c_{\text {grav }}^{\text {bulk }}, k_{R}^{b u l k}$ and $k_{L}^{b u l k}$ in section 3 except for the constant one loop shifts. This if we can show that $\Delta$ and $\delta$ vanish, then we would prove that the effect of the exterior contributions is to precisely cancel these constant shifts in the $A d S_{3}$ central charges.

We shall now show that $\Delta$ and $\delta$ vanish. For this we shall need to make some assumptions on the structure of the exterior modes. We make the following assumptions:

1. The exterior modes consist of free massless scalars and fermions belonging to singlet and/or spinors representations of $\mathrm{SU}(2)_{L}$ and $\mathrm{SU}(2)_{R}$.

2. The scalar modes which transform in the vector $(2,2)$ representation of the transverse rotation group $\mathrm{SO}(4)=\mathrm{SU}(2)_{L} \times \mathrm{SU}(2)_{R}$ are non-chiral. Physically this assumption stems from the fact that such modes arise from the oscillations of the center of mass mode of the black string which is non-chiral. Due to this assumption the contribution to the $\mathrm{SU}(2)_{R}$ and $\mathrm{SU}(2)_{L}$ anomalies from any scalar in the $\left(2_{L}, 2_{R}\right)$ representation of $\mathrm{SU}(2)_{L} \times \mathrm{SU}(2)_{R}$ always vanishes. Taking advantage of this fact we can assign the contribution to $\left(k_{L}, k_{R}\right)$ from a left-moving $\left(2_{L}, 2_{R}\right)$ scalar to be $(a, b)$ and a rightmoving $\left(2_{L}, 2_{R}\right)$ scalar to be $(-a,-b)$ for any arbitrary pair of numbers $(a, b)$. We shall choose $(a, b)=(-1,-1)$ for convenience.

To this we shall add the information that the $(1+1)$ dimensional conformal field theory of exterior modes is invariant under $(0,4)$ supersymmetry. This follows from the supersymmetry of the solution outside the $A d S_{3}$ region. We shall not make the assumption that the $\mathrm{SU}(2)$ R-symmetry current of this superconformal algebra has any relation to the spatial rotation group $\mathrm{SU}(2)_{R}$. Thus we shall not have any relation between $c_{R}^{\text {exterior }}$ and $k_{R}^{\text {exterior }}$.

We shall now separately evaluate the contribution to $\Delta$ and $\delta$ from each type of field that could appear as part of the exterior degrees of freedom. For this we need to calculate $k_{L}, k_{R}, c_{L}-c_{R}, c_{L, e f f}$ and $k_{L, e f f}$ from each field. This is done with the help of the following observations:

1. The calculation of $\left(k_{R}, k_{L}, c_{L}-c_{R}\right)$ is straighforward since these are given by the contribution to $\mathrm{SU}(2)_{L}, \mathrm{SU}(2)_{R}$ and gravitational anomalies.

2. The calculation of $c_{L, \text { eff }}$ and $k_{L, \text { eff }}$ involves computing the contribution from these fields to the index $\widetilde{C}_{2 k}^{\text {exterior }} \equiv \operatorname{Tr}(-1)^{2 J_{R}}\left(2 J_{R}\right)^{2} e^{2 \pi i p \tau+4 \pi i J_{L} z}$. To this end we note that the factor of $\left(2 J_{R}\right)^{2}$ is needed to soak up the $\mathrm{SU}(2)_{R}$ doublet fermion zero modes. Thus after taking the trace over the fermion zero modes we are left with $\operatorname{Tr}(-1)^{2 J_{R}} e^{2 \pi i p \tau+4 \pi i J_{L} z}$ from the oscillator modes. Due to supersymmetry this receives contribution only from the left-moving modes.

3. Since $(-1)^{2 J_{R}}=1$ for the $\mathrm{SU}(2)_{R}$ singlet fields, the $\mathrm{SU}(2)_{R}$ singlet left-moving fields contribute in the same way to the index and the degeneracy. Thus for them $c_{L, e f f}=$ $c_{L}$, and $k_{L, e f f}=k_{L}$.

4. $\mathrm{SU}(2)_{R}$ doublet left-moving fields have the property that the contribution to $\widetilde{C}_{2 k}^{\text {exterior }}$ from a left-moving scalar oscillator, given by $\left(1-e^{2 \pi i p_{o s c} \tau+4 \pi i J_{L, o s c} z}\right)^{-1}$, can be regarded as the inverse of the contribution to the partition function from a left-moving 
fermionic oscillator, and the contribution to $\widetilde{C}_{2 k}^{\text {exterior }}$ from a left-moving fermionic oscillator, given by $\left(1-e^{2 \pi i p_{o s c} \tau+4 \pi i J_{L, o s c} z}\right)$, can be regarded as the inverse of the contribution to the partition function from a left-moving bosonic oscillator. Thus their contribution to $c_{L, e f f}$ and $k_{L, e f f}$ can be computed by replacing the fermions by bosons and vice versa, and including an extra - sign in front of the corresponding values of $c_{L}$ and $k_{L}$.

This gives the following contribution to $\Delta$ and $\delta$ from various fields:

left-moving $\left(1_{L}, 1_{R}\right)$ scalar:

$$
k_{R}=0, \quad k_{L}=0, \quad c_{R}=0, \quad c_{L}=1, \quad c_{L, \text { eff }}=1, \quad k_{L, \text { eff }}=0, \quad \Delta=0, \quad \delta=0,
$$

left-moving $\left(2_{L}, 2_{R}\right)$ scalar:

$$
k_{R}=-1, \quad k_{L}=-1, \quad c_{R}=0, \quad c_{L}=4, \quad c_{L, e f f}=-2, \quad k_{L, \text { eff }}=-1, \quad \Delta=0, \quad \delta=0,
$$

left-moving $\left(2_{L}, 1_{R}\right)$ fermion:

$$
k_{R}=0, \quad k_{L}=\frac{1}{2}, \quad c_{R}=0, \quad c_{L}=1, \quad c_{L, e f f}=1, \quad k_{L, e f f}=\frac{1}{2}, \quad \Delta=0, \quad \delta=0,
$$

left-moving $\left(1_{L}, 2_{R}\right)$ fermion:

$$
k_{R}=-\frac{1}{2}, \quad k_{L}=0, \quad c_{R}=0, \quad c_{L}=1, \quad c_{L, e f f}=-2, \quad k_{L, \text { eff }}=0, \quad \Delta=0, \quad \delta=0,
$$

right-moving $\left(1_{L}, 1_{R}\right)$ scalar:

$$
k_{R}=0, \quad k_{L}=0, \quad c_{R}=1, \quad c_{L}=0, \quad c_{L, e f f}=0, \quad k_{L, e f f}=0, \quad \Delta=1, \quad \delta=0,
$$

right-moving $\left(2_{L}, 2_{R}\right)$ scalar:

$$
k_{R}=1, \quad k_{L}=1, \quad c_{R}=4, \quad c_{L}=0, \quad c_{L, e f f}=0, \quad k_{L, e f f}=0, \quad \Delta=-2, \quad \delta=-1,
$$

right-moving $\left(2_{L}, 1_{R}\right)$ fermion:

$$
k_{R}=0, \quad k_{L}=-\frac{1}{2}, \quad c_{R}=1, \quad c_{L}=0, \quad c_{L, e f f}=0, \quad k_{L, e f f}=0, \quad \Delta=1, \quad \delta=\frac{1}{2},
$$

right-moving $\left(1_{L}, 2_{R}\right)$ fermion:

$$
k_{R}=\frac{1}{2}, \quad k_{L}=0, \quad c_{R}=1, \quad c_{L}=0, \quad c_{L, e f f}=0, \quad k_{L, e f f}=0, \quad \Delta=-2, \quad \delta=0 .
$$

Note that in evaluating the contribution to $k_{L}$ and $k_{R}$ from the $\left(2_{L}, 2_{R}\right)$ scalars we have exploited the freedom of choice mentioned earlier. From this table we see that the leftmoving exterior modes do not contribute to $\Delta$ or $\delta$. On the other hand since we have 
supersymmetry acting on the right-movers, and since the supersymmetry generators are doublets of $\mathrm{SU}(2)_{R}$, a right-moving $\mathrm{SU}(2)_{R}$ doublet scalar must be accompanied by a pair of $\mathrm{SU}(2)_{R}$ singlet fermions and a right-moving $\mathrm{SU}(2)_{R}$ doublet fermion must be accompanied by a pair of $\mathrm{SU}(2)_{R}$ single scalars. ${ }^{15}$ From $(4.12)$ we see that the net contribution to $\Delta$ and $\delta$ still vanishes for such fields.

Using $\Delta=0$ and $\delta=0$ we get from (4.9) that

$$
c_{L, e f f}^{\text {macro }}=c_{\text {grav }}^{\text {asymp }}+6 k_{R}^{\text {asymp }}, \quad k_{L, \text { eff }}^{\text {macro }}=k_{L}^{\text {asymp }} .
$$

As already argued before, $c_{g r a v}^{a s y m p}+6 k_{R}^{a s y m p}$ and $k_{L}^{a s y m p}$ are given respectively by the same computation as $c_{L}^{b u l k}$ and $k_{L}^{b u l k}$ of section 3 except that the constant shifts are absent. This proves that the effect of the inclusion of the exterior contribution is to remove the constant term in the central charges due to one loop corrections. Note also that in (4.12) the values of $c_{L}$ and $c_{L, e f f}$ differ for several of the modes. Thus if we had focussed on the absolute degeneracy rather than the index then its growth will not be controlled solely by the anomaly coefficients since for the contribution due to the exterior modes $c_{L, \text { eff }}$ will now be replaced by $c_{L}$.

\section{Microscopic results}

In this section we shall examine the computation of the microscopic indices of certain black holes in four and five dimensions, and show that these agree with the results of explicit macroscopic calculations given in section 3 and section 4 .

\subsection{D1-D5-p system in type IIB on $K 3 \times S^{1}$}

In this section we shall examine in detail the microscopic formulæ for the index of the D1-D5-p system in type IIB string theory compactified on $K 3 \times S^{1}$ in various limits. We consider a system of 1 D5-brane wrapped on $K 3 \times S^{1}$ and $Q_{1}+1$ D1-branes wrapped on $S^{1}$, carrying $n$ units of left-moving momentum along $S^{1}$ and $\mathrm{SU}(2)_{L}$ angular momentum $J_{L}=J / 2$. Since a D5-brane wrapped on K3 carries -1 unit of D1-brane charge, $Q_{1}$ represents the physical D1-brane charge carried by this system. We consider the index:

$$
d_{\text {micro }}\left(n, Q_{1}, J\right) \equiv C_{2}\left(n, Q_{1}, J\right)=-\frac{1}{2 !} \operatorname{Tr}\left[(-1)^{2 J_{R}}\left(2 J_{R}\right)^{2}\right],
$$

where the trace is taken over all states carrying fixed $Q_{1}, n$ and $J_{L}=J / 2$ but different values of $J_{R}$. The partition function $Z_{5 D}(\rho, \sigma, v)$, defined through the relation

$$
Z_{5 D}(\rho, \sigma, v) \equiv \sum_{Q_{1}, n, J} e^{2 \pi i\left(\rho n+\sigma Q_{1}+v J\right)}(-1)^{J} d_{\text {micro }}\left(n, Q_{1}, J\right)
$$

\footnotetext{
${ }^{15}$ We emphasize that that this does not imply that $\mathrm{SU}(2)_{R}$ is the zero mode part of the right-moving R-symmetry current. As already remarked, the latter acts trivially on all the left-moving fields while the former has non-trivial action on some left-movers.
} 
is given by $[30,31]$

$$
\begin{aligned}
Z_{5 D}(\rho, \sigma, v)= & e^{-2 \pi i \sigma} \prod_{\substack{k, l, j \in \mathbb{Z} \\
k \geq 1, l \geq 0}}\left(1-e^{2 \pi i(\sigma k+\rho l+v j)}\right)^{-c\left(4 l k-j^{2}\right)} \\
& \times\left\{\prod_{\substack{l \geq 1 \\
l \geq 1}}\left(1-e^{2 \pi i(l \rho+v)}\right)^{-2}\left(1-e^{2 \pi i(l \rho-v)}\right)^{-2}\left(1-e^{2 \pi i l \rho}\right)^{4}\right\}(-1)\left(e^{\pi i v}-e^{-\pi i v}\right)^{2}
\end{aligned}
$$

where $c(u)$ are defined via the relations:

$$
\begin{aligned}
& F(\tau, z)=\sum_{j, n \in \mathbb{Z}} c\left(4 n-j^{2}\right) e^{2 \pi i n \tau+2 \pi i j z} . \\
& F(\tau, z)=8\left[\frac{\vartheta_{2}(\tau, z)^{2}}{\vartheta_{2}(\tau, 0)^{2}}+\frac{\vartheta_{3}(\tau, z)^{2}}{\vartheta_{3}(\tau, 0)^{2}}+\frac{\vartheta_{4}(\tau, z)^{2}}{\vartheta_{4}(\tau, 0)^{2}}\right] .
\end{aligned}
$$

The first line of (5.3) is the contribution from the relative motion between the D1 and D5 branes [30] and the second line represents the contribution from the center of mass modes [51]. Strictly speaking we should subtract from this the contribution from the half-BPS states carrying zero momentum, but as long as we use this formula to extract the index of states carrying non-zero momentum along $S^{1}$, we shall not make any error. The $-\left(2 J_{R}\right)^{2} / 2$ ! factor in the trace has been absorbed by the four fermion zero modes associated with the center of mass motion carrying $\left(J_{L}, J_{R}\right)=\left(0, \pm \frac{1}{2}\right)$, and the factor of $-\left(e^{\pi i v}-e^{-\pi i v}\right)^{2}$ comes from the contribution from the four fermion zero modes on the D1-D5 world-volume carrying $\left(J_{L}, J_{R}\right)=\left( \pm \frac{1}{2}, 0\right)$.

Eq. (5.3) may be rewritten as

$$
Z_{5 D}(\rho, \sigma, v)=-\left(e^{\pi i v}-e^{-\pi i v}\right)^{4} \frac{\eta(\rho)^{24}}{\Phi_{10}(\rho, \sigma, v)},
$$

where

$$
\Phi_{10}(\rho, \sigma, v)=e^{2 \pi i \sigma+2 \pi i \rho+2 \pi i v} \prod_{\substack{k, l, j \in \mathbb{Z} \\ k, l \geq 0, j<\text { for } k=l=0}}\left(1-e^{2 \pi i(\sigma k+\rho l+v j)}\right)^{c\left(4 l k-j^{2}\right)},
$$

is the Igusa cusp form. In going from (5.3) to (5.6) we have used $c(0)=20, c(-1)=2$. From (5.2), (5.6) we get

$$
d_{\text {micro }}\left(n, Q_{1}, J\right)=(-1)^{J+1} \int_{0}^{1} d \rho \int_{0}^{1} d \sigma \int_{0}^{1} d v\left(e^{\pi i v}-e^{-\pi i v}\right)^{4} e^{-2 \pi i\left(\rho n+\sigma Q_{1}+J v\right)} \frac{\eta(\rho)^{24}}{\Phi_{10}(\rho, \sigma, v)} .
$$

We shall be interested in studying the behavior of $d_{\text {micro }}\left(n, Q_{1}, J\right)$ in two different limits:

1. Type IIB Cardy limit: $n$ large at fixed $Q_{1}$ and $Q_{1}-\frac{J^{2}}{4 n}>K_{1}$ for some fixed positive number $K_{1}$.

2. Type IIA Cardy limit [68]: $Q_{1}$ large at fixed $n$ and $n-\frac{J^{2}}{4 Q_{1}}>K_{2}$ for some fixed positive number $K_{2}$. 
Estimates for $K_{1}, K_{2}$ can be found in appendix B. In both these limits the combination $\Delta \equiv\left(4 Q_{1} n-J^{2}\right)$ becomes large. In this case the asymptotic expansion of $d_{\text {micro }}\left(n, Q_{1}, J\right)$ is governed by the residue of the integrand in (5.8) on the subspace $[1,2,9,18]$

$$
\rho \sigma-v^{2}+v=0
$$

where the integrand has a pole. Since the analysis in $[1,2,9,18]$ were carried out in a different limit where $n, Q_{1}$ and $J$ were all large and of same order, we have given a careful analysis in appendix B showing that even in the two limits we are considering the dominant contribution comes from this pole. Near this pole

$$
\frac{1}{\Phi_{10}(\rho, \sigma, v)}=-\left(4 \pi^{2}\right)^{-1} \rho^{10} \check{v}^{-2} \eta(\check{\rho})^{-24} \eta(\check{\sigma})^{-24}+\text { non-singular },
$$

where

$$
\check{\rho}=\frac{\rho \sigma-v^{2}}{\rho}, \quad \check{\sigma}=\frac{\rho \sigma-(v-1)^{2}}{\rho}, \quad \check{v}=\frac{\rho \sigma-v^{2}+v}{\rho} .
$$

Picking up the residue at the pole at (5.9) restricts the three dimensional integral to a two dimensional subspace. This is best done by changing the variables of integration to $(\check{\rho}, \check{\sigma}, \check{v})$, and using

$$
d \rho \wedge d \sigma \wedge d v=-(2 \check{v}-\check{\rho}-\check{\sigma})^{-3} d \check{\rho} \wedge d \check{\sigma} \wedge d \check{v} .
$$

In these variables the residue at the pole at $\check{v}=0$ can be calculated easily using standard procedure. Introducing the variables $\left(\tau_{1}, \tau_{2}\right)$ via

$$
\check{\rho}=\tau_{1}+i \tau_{2}, \quad \check{\sigma}=-\tau_{1}+i \tau_{2},
$$

we have near the $\breve{v}=0$ subspace:

$\rho=\frac{i}{2 \tau_{2}}+\frac{1}{2 \tau_{2}^{2}} \check{v}+\mathcal{O}\left(\check{v}^{2}\right), \quad \sigma=i \frac{\tau_{1}^{2}+\tau_{2}^{2}}{2 \tau_{2}}+\frac{\tau_{1}^{2}+\tau_{2}^{2}}{2 \tau_{2}^{2}} \check{v}+\mathcal{O}\left(\check{v}^{2}\right), \quad v=\frac{1}{2}-i \frac{\tau_{1}}{2 \tau_{2}}-\frac{\tau_{1}}{2 \tau_{2}^{2}} \check{v}+\mathcal{O}\left(\check{v}^{2}\right)$.

Then the contribution to the integral from the residue at $\check{v}=0$ is given by $[2,9,18]^{16}$

$$
d_{\text {micro }}\left(n, Q_{1}, J\right) \simeq \int \frac{d^{2} \tau}{\tau_{2}^{2}} e^{-F\left(\tau_{1}, \tau_{2}\right)},
$$

where

$$
\begin{aligned}
F\left(\tau_{1}, \tau_{2}\right)= & -\frac{\pi}{\tau_{2}}\left[n+Q_{1}\left(\tau_{1}^{2}+\tau_{2}^{2}\right)-\tau_{1} J\right]+24 \ln \eta\left(\tau_{1}+i \tau_{2}\right)+24 \ln \eta\left(-\tau_{1}+i \tau_{2}\right) \\
& +12 \ln \left(2 \tau_{2}\right)-24 \ln \eta\left(\frac{i}{2 \tau_{2}}\right)-4 \ln \left\{2 \cosh \left(\frac{\pi \tau_{1}}{2 \tau_{2}}\right)\right\} \\
& -\ln \left[\frac{1}{4 \pi}\left\{26+\frac{2 \pi}{\tau_{2}}\left(n+Q_{1}\left(\tau_{1}^{2}+\tau_{2}^{2}\right)-\tau_{1} J\right)+i \frac{24}{\tau_{2}} \frac{\eta^{\prime}\left(i / 2 \tau_{2}\right)}{\eta\left(i / 2 \tau_{2}\right)}+4 \pi \frac{\tau_{1}}{\tau_{2}} \tanh \frac{\pi \tau_{1}}{2 \tau_{2}}\right\}\right] .
\end{aligned}
$$

\footnotetext{
${ }^{16}$ In $[2,9,18]$ the analysis was carried out for the four dimensional black hole for which the integrand in (5.8) involves $1 / \Phi_{10}$ instead of $\eta(\rho)^{24} / \Phi_{10}$. Eqs. (5.15), (5.16) are obtained by multiplying the integrand of $[2,9,18]$ by a factor of $\eta(\rho)^{24}$, and then picking up the residue at $\check{v}=0$. This procedure is similar to the ones followed in [68,69], except that we have included in our analysis the contribution from the center of mass degrees of freedom of the D1-D5-brane system and removed the contribution due to the fermion zero modes associated with the hair.
} 
$\simeq$ in (5.15) implies equality up to exponentially suppressed contributions. Although we have not been careful to keep track of the sign, this can be done by carefully following each step as in [18]. The result is that the $\tau_{1}, \tau_{2}$ integrations run along the imaginary $\tau_{1}, \tau_{2}$ directions through the saddle points of $F\left(\tau_{1}, \tau_{2}\right)$ and the integration measure $d^{2} \tau$ represents $d\left(I m \tau_{1}\right) d\left(I m \tau_{2}\right)$. Thus the leading contribution to $d_{m i c r o}\left(n, Q_{1}, J\right)$ is positive.

The integration over $\tau_{1}, \tau_{2}$ can be evaluated using the method of steepest descent. First of all note that if we ignore all terms except the one inside the first square bracket on the right hand side of $(5.16)$, the extremum of $F\left(\tau_{1}, \tau_{2}\right)$ lies at

$$
\tau_{1}=\frac{J}{2 Q_{1}}, \quad \tau_{2}=\sqrt{\frac{4 n Q_{1}-J^{2}}{4 Q_{1}^{2}}} .
$$

If $Q_{1}, n$ and $J$ become large at the same rate then $\left(\tau_{1}, \tau_{2}\right)$ are of order unity and the first term in the square bracket in (5.16) dominates over the other term. However since we want to take different limits we need to keep track of the contribution from the rest of the terms.

1. In the type IIB Cardy limit we have $n \rightarrow \infty$ at fixed values of $Q_{1}$, and $Q_{1}-\frac{J^{2}}{4 n}>K_{1}$. In this case we get from (5.17) $\tau_{2} \sim \sqrt{n}$ and $\tau_{1}<\sqrt{n}$. Since $\tau_{2}$ is large, we have

$$
\begin{aligned}
24 \ln \eta\left(\tau_{1}+i \tau_{2}\right) & \simeq 2 \pi i\left(\tau_{1}+i \tau_{2}\right), \quad 24 \ln \eta\left(-\tau_{1}+i \tau_{2}\right) \simeq 2 \pi i\left(-\tau_{1}+i \tau_{2}\right), \\
24 \ln \eta\left(\frac{i}{2 \tau_{2}}\right) & \simeq-4 \pi \tau_{2} .
\end{aligned}
$$

Substituting this into (5.16) we see that in the rest of the terms other than those contained in the first square bracket the terms linear in $\tau_{1}$ and $\tau_{2}$ cancel, and at (5.17) the net contribution from these terms is small compared to the first term in the square bracket. Thus the leading contribution to $-\ln d_{\text {micro }}$ will be obtained by evaluating the first term in the square bracket at the saddle point (5.17). This gives

$$
\ln d_{\text {micro }}\left(n, Q_{1}, J\right) \simeq \pi \sqrt{4 n Q_{1}-J^{2}} .
$$

In this equation $\simeq$ denotes equality up to power suppressed corrections. In the rest of this section $\simeq$ in the expression for $d_{\text {micro }}$ will denote corrections suppressed by powers of $n\left(Q_{1}\right)$ in the type IIB Cardy (type IIA Cardy) limit. In principle we can compute these power suppressed corrections by systematically carrying out the integration over $\left(\tau_{1}, \tau_{2}\right)$ about this saddle point.

If we have $Q_{5}$ D5-branes instead of one D5-brane with $\operatorname{gcd}\left(Q_{1}, Q_{5}\right)=1$ then by duality invariance the result for the index depends on the combination $Q_{1} Q_{5}$. Thus the result for general $Q_{5}$ is obtained by replacing $Q_{1}$ by $Q_{1} Q_{5}$ in (5.19):

$$
\ln d_{\text {micro }}\left(n, Q_{1}, Q_{5}, J\right) \simeq \pi \sqrt{4 n Q_{1} Q_{5}-J^{2}} .
$$

The result is valid for large $n$ with $Q_{1} Q_{5}-\frac{J^{2}}{4 n}>K_{1}$. This result is in perfect agreement with the result of the direct macroscopic calculation given in (3.16).

It is worth comparing the result for the index with the result for the degeneracy. For simplicity we shall sum over all the $J$ values keeping the other charges fixed. In this 
case the index grows as $\exp \left[\pi \sqrt{4 n Q_{1} Q_{5}}\right]$. For computing the degeneracy we shall apply the Cardy formula. Since the relative motion of the D1-D5 system is described by a super-conformal field theory whose target space is the symmetric product of $\left(Q_{1} Q_{5}+1\right)$ copies of $K 3$, we get a central charge of $6\left(Q_{1} Q_{5}+1\right)$ from the dynamics of these modes. The center of mass motion in the transverse directions will give a superconformal field theory with target space $R^{4}$, and gives a central charge 6 . Thus the total central charge of this system is $c^{\text {micro }}=6\left(Q_{1} Q_{5}+2\right)$, both for the left and the right-moving modes. Since the black hole microstates are identified as the left-moving excitations in this CFT, we get the expected growth of degeneracy to be $\exp \left[2 \pi \sqrt{c^{\text {micro }} / 6}\right] \sim \exp \left[2 \pi \sqrt{\left(Q_{1} Q_{5}+2\right) n}\right]$. This is different from the rate of growth $\exp \left[2 \pi \sqrt{n Q_{1} Q_{5}}\right]$ of the index.

2. In the type IIA Cardy limit we have $Q_{1} \rightarrow \infty$ at fixed values of $n$, and $n-\frac{J^{2}}{4 Q_{1}}>K_{2}$. Thus (5.17) gives $\tau_{2} \sim 1 / \sqrt{Q_{1}}$ and $\tau_{1} \sim 1 / \sqrt{Q_{1}}$. Since $\left(\tau_{1}+i \tau_{2}\right)$ is small, it is natural to define

$$
\pm \sigma_{1}+i \sigma_{2}=-\frac{1}{ \pm \tau_{1}+i \tau_{2}} .
$$

At (5.17), $\sigma_{2}=\sqrt{4 n Q_{1}-J^{2}} / 2 n$. This is large in the limit we are considering, and hence we have

$$
\begin{aligned}
24 \ln \eta\left(\tau_{1}+i \tau_{2}\right) & \simeq 2 \pi i\left(\sigma_{1}+i \sigma_{2}\right), \quad 24 \ln \eta\left(-\tau_{1}+i \tau_{2}\right) \simeq 2 \pi i\left(-\sigma_{1}+i \sigma_{2}\right), \\
24 \ln \eta\left(\frac{i}{2 \tau_{2}}\right) & \simeq-\frac{\pi\left(\sigma_{1}^{2}+\sigma_{2}^{2}\right)}{\sigma_{2}} .
\end{aligned}
$$

Each of these terms is of order $\sqrt{Q_{1}}$ at the saddle point and they do not cancel. Since in the limit of large $Q_{1}$, the terms inside the first square bracket of (5.16) and the contribution from the rest of the terms are both of order $\sqrt{Q_{1}}$, it is no longer appropriate to neglect the rest of the terms. Instead we must evaluate the saddle point by taking into account the contribution from all the terms. We shall proceed with the ansatz that at the saddle point $\sigma_{2}$ is of order $\sqrt{Q_{1}}$; this will be verified at the end to check the self-consistency of our approximation. With this assumption we can approximate the $\eta$ functions by $(5.22)$ and get the leading terms in $F\left(\tau_{1}, \tau_{2}\right)$ to be:

$$
-\frac{\pi}{\sigma_{2}}\left[Q_{1}+n\left(\sigma_{1}^{2}+\sigma_{2}^{2}\right)+\sigma_{1} J\right]-4 \pi \sigma_{2}+\frac{\pi\left(\sigma_{1}^{2}+\sigma_{2}^{2}\right)}{\sigma_{2}} .
$$

This has an extremum at

$$
\sigma_{1}=-\frac{J}{2(n-1)}, \quad \sigma_{2}=\sqrt{\left(Q_{1}-\frac{J^{2}}{4(n-1)}\right) /(n+3)},
$$

and at this extremum

$$
F=-2 \pi \sqrt{(n+3)\left(Q_{1}-\frac{J^{2}}{4(n-1)}\right)} .
$$


This gives

$$
\ln d_{\text {micro }}\left(n, Q_{1}, J\right) \simeq 2 \pi \sqrt{(n+3)\left(Q_{1}-\frac{J^{2}}{4(n-1)}\right)},
$$

up to power suppressed corrections. Furthermore from (5.24) we see that $\sigma_{2} \sim \sqrt{Q_{1}}$ in agreement with our ansatz.

We can write down the result for $Q_{5}$ number of D5-branes with $\operatorname{gcd}\left\{Q_{1}, Q_{5}\right\}=1$ by replacing $Q_{1}$ by $Q_{1} Q_{5}$ in (5.26):

$$
\ln d_{\text {micro }}\left(n, Q_{1}, Q_{5}, J\right) \simeq 2 \pi \sqrt{(n+3)\left(Q_{1} Q_{5}-\frac{J^{2}}{4(n-1)}\right)} .
$$

This result is valid when $Q_{1} Q_{5}$ is large, and $n-\frac{J^{2}}{4 Q_{1} Q_{5}}>K_{2}$. This is again in perfect agreement with the result of the macroscopic calculation given in (3.29).

To first subleading order in an expansion in powers of $1 / n$ and $J^{2}$ this agreement was found in [68].

\subsection{D1-D5-p-KK monopole system in type IIB on $K 3 \times T^{2}$}

We consider now the same D1-D5-p system analyzed in section 5.1 and place it at the center of a Taub-NUT space. This gives a four dimensional black hole, with the asymptotic circle $\widetilde{S}^{1}$ of the Taub-NUT space identified as a new compact direction. Since the black hole breaks 12 of the 16 supersymmetries of the theory, the relevant index is $B_{6}$. The TaubNUT background has three effects on the index computation: it first of all converts the angular momentum $2 J_{L}=J$ to momentum along $\widetilde{S}^{1}[98]$, it shifts the momentum along $S^{1}$ by -1 units, and it gives additional contribution to the 'partition function' for the index [9]. We shall denote by $d_{\text {micro }}\left(n, Q_{1}, J\right)$ the negative of the sixth helicity trace index for these dyons. Then $[1-3,9]$

$$
d_{\text {micro }}\left(n, Q_{1}, J\right)=(-1)^{J+1} \int_{0}^{1} d \rho \int_{0}^{1} d \sigma \int_{0}^{1} d v e^{-2 \pi i\left(\rho n+\sigma Q_{1}+J v\right)} \frac{1}{\Phi_{10}(\rho, \sigma, v)} .
$$

We shall be interested in the behavior of this quantity in the limit of large $n$ at fixed values of $Q_{1}$, and $J=0$. The analysis proceeds as in section 5.1 and we arrive at the result $[2,9,18]$ :

$$
d_{\text {micro }}\left(n, Q_{1}, J=0\right) \simeq \int \frac{d^{2} \tau}{\tau_{2}^{2}} e^{-F\left(\tau_{1}, \tau_{2}\right)}
$$

where

$$
\begin{aligned}
F\left(\tau_{1}, \tau_{2}\right)= & -\frac{\pi}{\tau_{2}}\left[n+Q_{1}\left(\tau_{1}^{2}+\tau_{2}^{2}\right)\right]+24 \ln \eta\left(\tau_{1}+i \tau_{2}\right)+24 \ln \eta\left(-\tau_{1}+i \tau_{2}\right) \\
& +12 \ln \left(2 \tau_{2}\right)-\ln \left[\frac{1}{4 \pi}\left\{26+\frac{2 \pi}{\tau_{2}}\left(n+Q_{1}\left(\tau_{1}^{2}+\tau_{2}^{2}\right)\right)\right\}\right] .
\end{aligned}
$$


Using $\tau_{1} \rightarrow-\tau_{1}$ symmetry we can set $\tau_{1}=0$ at the saddle point. To extract the behavior of this integral for large $n$ we shall proceed with the ansatz that $\tau_{2}$ is large, of order $\sqrt{n}$ at the saddle point. In this case we can approximate $F\left(\tau_{1}=0, \tau_{2}\right)$ by

$$
F\left(\tau_{1}=0, \tau_{2}\right)=-\frac{\pi}{\tau_{2}}\left[n+Q_{1} \tau_{2}^{2}\right]-4 \pi \tau_{2} .
$$

This has an extremum at

$$
\tau_{2}=\sqrt{n /\left(Q_{1}+4\right)} .
$$

Thus at the extremum $\tau_{2} \sim \sqrt{n}$, satisfying our ansatz. Evaluating $F\left(0, \tau_{2}\right)$ at the extremum we get

$$
\ln \left(d_{\text {micro }}\left(n, Q_{1}, J=0\right)\right) \simeq-\left.F\left(0, \tau_{2}\right)\right|_{\text {extremum }}=2 \pi \sqrt{\left(Q_{1}+4\right) n} .
$$

We can in fact find the full asymptotic expansion by replacing the $-12 \ln \left(2 \tau_{2}\right)$ $+\ln \left[\frac{1}{4 \pi}\left\{26+\frac{2 \pi}{\tau_{2}}\left(n+Q_{1}\left(\tau_{1}^{2}+\tau_{2}^{2}\right)\right)\right\}\right]$ factor in the exponent by a multiplicative factor of $\left(2 \tau_{2}\right)^{-12}\left[\frac{1}{4 \pi}\left\{26+\frac{2 \pi}{\tau_{2}}\left(n+Q_{1}\left(\tau_{1}^{2}+\tau_{2}^{2}\right)\right)\right\}\right]$ in the integrand and approximating $\eta(\tau)$ by $e^{2 \pi i \tau / 24}$ as in (5.31). The $\tau_{1}$ integral then becomes a gaussian integral which can be evaluated, and the $\tau_{2}$ integral gives sum of Bessel functions. Using appropriate identities among Bessel functions we can bring the integral to the form

$$
d_{\text {micro }}=C_{0}\left(\frac{n}{Q_{1}+4}\right)^{-23 / 4} I_{23 / 2}\left(2 \pi \sqrt{n\left(Q_{1}+4\right)}\right),
$$

where $C_{0}$ is a constant independent of $n$ and $I_{\nu}$ denotes the standard Bessel function with imaginary argument. This is precisely the leading term in the Rademacher expansion [99].

The final answer (5.34) can be readily determined directly using standard facts about the Rademacher expansion of modular forms and Jacobi forms as follows. Doing the $\sigma$ integral first, we pick up the $Q_{1}$-th Fourier coffecient of the partition function. Since $1 / \Phi_{10}$ is a Siegel modular form of weight -10 , this Fourier coefficient $\psi(\tau, z)$ is a weak Jacobi form in two variables of weight -10 and index $Q_{1}$. Furthermore, $\psi$ is known to be the partition function of a $(0,4)$ SCFT of central charge $C=6 Q_{1}+24$. For a Jacobi form of weight $-k$, the index of the Bessel function and the power of the prefactor in the Rademacher expansion $^{17}$ is controlled by $(k+3 / 2)$ which in our case is $23 / 2$. The argument of the Bessel function and the prefactor are, on the other hand, given by $2 \pi \sqrt{C n / 6}$ which in our case gives $2 \pi \sqrt{n\left(Q_{1}+4\right)}$.

If we take a system with $Q_{5}$ D5-branes instead of a single D5-brane with $\operatorname{gcd}\left(Q_{1}, Q_{5}\right)=$ 1 then the $B_{6}$ index must depend on $Q_{1}$ and $Q_{5}$ through the duality invariant combination $Q_{1} Q_{5}$. This gives

$$
\ln \left(d_{\text {micro }}\left(n, Q_{1}, Q_{5}, J=0\right)\right) \simeq 2 \pi \sqrt{\left(Q_{1} Q_{5}+4\right) n} .
$$

What if we have $K$ KK-monopoles instead of a single KK monopole associated with $\widetilde{S}^{1}$ ? As long as $\operatorname{gcd}\left(Q_{1}, Q_{5}\right)=1$ and $\operatorname{gcd}(n, K)=1$, we can find a duality transformation that

\footnotetext{
${ }^{17}$ The usual Rademacher expansion of weak Jacobi forms assumes that the Jacobi form is holomorphic. In our case, turns out to be meromorphic because of the poles in partition function and the Rademacher expansion is modified but by terms that exponentially subleading [99].
} 
maps this charge vector to the one considered above with $n$ replaced by $n K[100,101]$. Thus we have

$$
\ln d_{\text {micro }}\left(n, Q_{1}, Q_{5}, K, J=0\right) \simeq 2 \pi \sqrt{\left(Q_{1} Q_{5}+4\right) n K} .
$$

This is in perfect agreement with the macroscopic result (3.35), computed by describing the system as a black hole in M-theory on $K 3 \times T^{3}$, carrying M5-brane charges and momentum along a circle.

When the above arithmetic condition on $\left(n, K, Q_{1}, Q_{5}\right)$ fails to hold there is no duality transformation that maps this charge vector to the one for which we carried out the analysis. Nevertheless the answer for $B_{6}$ for these more general charge vectors is known [19-21] and, in the limit of large $n$, differs from (5.36) by exponentially suppressed terms. Thus we can continue to use (5.36) for the general dyon.

\subsection{Black holes in toroidally compactified type II string theory}

In this section we shall generalize the analysis of the previous sections to toroidally compactified type IIB string theory. Since the D1-D5-p system on $T^{4} \times S^{1}$ describes a $1 / 8$ BPS state in a theory with 32 unbroken supercharges, the relevant index is $C_{6}$ defined in (2.3). This index was computed in [31]. For simplicity we shall set $Q_{5}=1$ and denote the corresponding index $C_{6}\left(n, Q_{1}, J\right)$ by $d_{\text {micro }}\left(n, Q_{1}, J\right)$; at the end we can recover the result for general $Q_{5}$ satisfying $\operatorname{gcd}\left(Q_{1}, Q_{5}\right)=1$ by replacing $Q_{1}$ by $Q_{1} Q_{5}$. The result of [31] for the index may be expressed as

$$
\sum_{J}(-1)^{J} d_{\text {micro }}\left(n, Q_{1}, J\right) e^{2 \pi i J v}=\left(e^{i \pi v}-e^{-i \pi v}\right)^{4} \sum_{j \in \mathbb{Z}} \sum_{s \mid n, Q_{1}, j} s \widehat{c}\left(\frac{4 Q_{1} n-j^{2}}{s^{2}}\right) e^{2 \pi i v j}
$$

where $\widehat{c}(\Delta)$ is defined through the relation:

$$
-\vartheta_{1}(z \mid \tau)^{2} \eta(\tau)^{-6} \equiv \sum_{k, l} \widehat{c}\left(4 k-l^{2}\right) e^{2 \pi i(k \tau+l z)} .
$$

$\vartheta_{1}(z \mid \tau)$ and $\eta(\tau)$ are respectively the odd Jacobi theta function and the Dedekind eta function. The $(-1)^{J}$ factor in (5.37) appears from the inclusion of an extra $(-1)^{J}$ factor in the definition of the index in [31]. In the limit when $Q_{1} n$ is large only the $s=1$ term is important and we get

$$
d_{\text {micro }}\left(n, Q_{1}, J\right) \simeq(-1)^{J+1} \int_{0}^{1} d \tau \int_{0}^{1} d v e^{-2 \pi i Q_{1} n \tau-2 \pi i J v}\left(e^{\pi i v}-e^{-\pi i v}\right)^{4} \frac{\vartheta_{1}(v \mid \tau)^{2}}{\eta(\tau)^{6}}
$$

up to exponentially suppressed corrections. We shall evaluate the integral over $\tau$ and $v$ using the saddle point method. We proceed with the ansatz that at the saddle point $\tau$ is small and $v \sim 1$, and verify this at the end. In this case we can express the integrand in $(5.39)$ as

$$
(-1)^{J} e^{-2 \pi i Q_{1} n \tau-2 \pi i J v}\left(e^{\pi i v}-e^{-\pi i v}\right)^{4} e^{-2 \pi i v^{2} / \tau} e^{2 \pi i v / \tau}\left(1-e^{-2 i \pi v / \tau}\right)^{2}(-i \tau)^{2} .
$$


Extremizing the integrand with respect to $v$ and $\tau$ we find the approximate saddle point in the rangle $0 \leq \operatorname{Re}(v)<1$ at

$$
v=\frac{1}{2}-\frac{J}{2} \tau+\cdots, \quad \tau=i / \sqrt{4 n Q_{1}-J^{2}}+\cdots,
$$

where $\cdots$ denote subleading terms. The value of the integrand at this saddle point is

$$
\exp \left[\pi \sqrt{4 n Q_{1}-J^{2}}+\cdots\right]
$$

This gives the leading contribution to $d_{\text {micro }}\left(n, Q_{1}, J\right)$. We can recover the results for $Q_{5} \neq 1$ with $\operatorname{gcd}\left(Q_{1}, Q_{5}\right)=1$ by replacing $Q_{1}$ by $Q_{1} Q_{5}$ in (5.42). This gives

$$
\ln d_{\text {micro }}\left(n, Q_{1}, Q_{5}, J\right) \simeq \pi \sqrt{4 n Q_{1} Q_{5}-J^{2}} .
$$

This is in perfect agreement with the macroscopic result given in (3.36) and (3.37). Note that in the microscopic analysis there is no distinction between type IIB Cardy limit $(n \rightarrow$ $\infty)$ and type IIA Cardy limit $\left(Q_{1} \rightarrow \infty\right)$ since the result depends on the combination $Q_{1} n$.

If instead of using the index we had computed the absolute degeneracy then the results would change as follows. The motion of $Q_{1}$ D1-branes inside a single D5-brane gives us $4 Q_{1}$ bosonic degrees of freedom and their $4 Q_{1}$ fermionic partners. Besides this we have four extra bosonic modes associated with the D1-D5 center of mass motion and four more bosonic modes associated with the Wilson lines on the D5-brane along $T^{4}$. Thus we have eight extra bosonic modes and their fermionic superpartners. This would give a total contribution of $6\left(Q_{1}+2\right)$ to the left-handed central charge, and the logarithm of the degeneracy computed from this would grow as $\pi \sqrt{4 n\left(Q_{1}+2\right)}$ for $J=0$. This is clearly different from (5.42) for $J=0$.

Finally consider the four dimensional system containing $Q_{5}$ D5-branes along $T^{4} \times S^{1}$, $Q_{1}$ D1-branes along $S^{1}$ and K Kaluza-Klein monopoles associated with $\widetilde{S}^{1}$, carrying $n$ units of momentum along $S^{1}$. This is U-dual to the M5-brane configuration discussed in section 3.3. We shall restrict our analysis to the case $\operatorname{gcd}\left\{K n, Q_{1} Q_{5}, K Q_{1}, K Q_{5}, n Q_{1}, n Q_{5}\right\}=$ 1. The exact $B_{14}$ index of these states is known, and up to exponentially suppressed corrections, the index is given by $[32,34,35]$

$$
-B_{14} \simeq-\widehat{c}\left(4 Q_{1} Q_{5} K n\right)
$$

with $\widehat{c}(\Delta)$ defined as in (5.37). For large $\Delta$ we have [29]

$$
\widehat{c}(\Delta) \sim(-1)^{\Delta+1} \Delta^{-2} \exp (\pi \sqrt{\Delta}) .
$$

eq.(5.44) now shows that the logarithm of the index $-B_{14}$ grows as $2 \pi \sqrt{Q_{1} Q_{5} K n}$. This gives the microscopic prediction for the logarithm of the index of the four dimensional black hole:

$$
\ln d_{\text {micro }}\left(n, Q_{1}, Q_{5}, K\right) \simeq 2 \pi \sqrt{Q_{1} Q_{5} K n} .
$$

This is in perfect agreement with the macroscopic result given in (3.38). 


\section{MSW analysis for M5-branes on $K 3 \times T^{3}$ and $T^{7}$}

In section 3.2 we described a black hole whose microscopic description contains M5-branes wrapped on a 5 -cycle of $K 3 \times T^{3}$ or $T^{7}$. However while computing the microscopic index of this system in section 5.2 we used an indirect method by mapping it to a D1-D5-p-KK monopole system in type IIB string theory. In this section we shall directly compute the microscopic index of the M5-brane system following [102], and show that the results agree with those obtained in section 5.2.

\subsection{M5-brane on $K 3 \times T^{3}$}

We begin by recalling the system of M5-branes described in section 3.2. We consider Mtheory on $K 3 \times S^{1} \times \widehat{S}^{1} \times S_{M}^{1}$, and take a brane configuration consisting of $Q_{1}$ M5-branes along $C_{2} \times S^{1} \times \widehat{S}^{1} \times S_{M}^{1}, Q_{5}$ M5-branes wrapped along $\widetilde{C}_{2} \times S^{1} \times \widehat{S}^{1} \times S_{M}^{1}$, and $K$ M5-branes wrapped along $K 3 \times S^{1}$, carrying $n$ units of momentum along $S^{1}$. The $B_{6}$ index of this configuration can be calculated following the procedure described in [41, 102]. In order to follow the notation of [102], we introduce some new notation for the charges, denoting the electric charges by $\left(q_{0}, q_{a}\right)$ and magnetic charges by $\left(p^{0}, p^{a}\right)$. The charge $q_{0}$ corresponds to momentum along the circle $S^{1}$ while $q_{a}$ corresponds to exciting the self-dual antisymmetric tensor field on the 5-brane, carrying charges corresponding to wrapping M2-branes on various 2-cycles of $K 3 \times \widehat{S}^{1} \times S_{M}^{1}$. The magnetic charge $p^{0}$ corresponds to a Kaluza-Klein monopole associated with the circle $S^{1}$. The other magnetic charges are associated with an M5-brane wrapping $P \times S^{1}$ with $P$ a four cycle of $K 3 \times \widehat{S}^{1} \times S_{M}^{1}$. For the configuration we are considering, $p^{0}$ and $q_{a}$ for $a \neq 0$ vanish, the charges $p^{a}$ can be identified with the triplet $\left(Q_{1}, Q_{5}, K\right)$ and the charge $q_{0}$ can be identified with $n$. Using the isomorphism between 4-cycles and 2-forms we can associate with $P$ a 2 -form on $M$ which we shall also denote by $P$. In this case we can write the magnetic charge vector in cohomology language, i.e, $P=p^{a} \Sigma_{a}$ with $\Sigma_{a} \in H^{2}(M, \mathbb{Z}), M \equiv K 3 \times \widehat{S}^{1} \times S_{M}^{1}$.

If we take the limit in which the circle $S^{1}$ has a size much larger than the size of $K 3 \times \widehat{S}^{1} \times S_{M}^{1}$, then the low energy limit of the effective theory describing the dynamics of the 5-brane on $P \times S^{1}$ is a two dimensional $(0,4)$ CFT. The BPS states in this theory involve left-moving excitations and the growth of degeneracy of these states for large momentum is determined in terms of the left-moving central charge $c_{L}^{\text {micro }}$ via the Cardy formula. $c_{L}^{\text {micro }}$ in turn is given by $N_{L}^{B}+\frac{1}{2} N_{L}^{F}$ where $N_{L}^{B}$ and $N_{L}^{F}$ are the numbers of left-handed bosons and fermions respectively. If instead of the degeneracy we consider the helicity trace index $B_{6}$, then the computation proceeds as follows. The requirement of unbroken supersymmetry forces the right-movers into their ground state. The $(2 h)^{6}$ factor in the trace is soaked up by the 12 fermion zero modes associated with the broken supersymmetry generators. Thus we are left with the trace over the left-handed bosonic and fermionic non-zero mode oscillators, weighted by $(-1)^{F}$ where $F$ denotes fermion number. The growth of this trace for large momentum along $S^{1}$ is controlled by a Cardy like formula, but with an effective central charge

$$
c_{L, \text { eff }}^{\text {micro }}=N_{L}^{B}-N_{L}^{F}
$$


This follows from the fact that the insertion of $(-1)^{F}$ into the trace does not affect the contribution to the partition function due to a bosonic oscillator, but the contribution to the partition function due to a fermion is now given by the inverse of the contribution from a boson. Note that if $N_{L}^{F}=0$ then $c_{L, e f f}^{\text {micro }}=c_{L}^{\text {micro }}$, but otherwise they are different.

Now the numbers of left and right-moving bosons are given by [102]

$$
\begin{aligned}
& N_{L}^{B}=d_{p}(P)+b_{2}^{-}(P)+3, \\
& N_{R}^{B}=d_{p}(P)+b_{2}^{+}(P)+3 .
\end{aligned}
$$

Here $d_{p}$ is the dimension the moduli space of deformations of $P$ inside $M, 3$ accounts for the center of mass translations and $b_{2}^{-}, b_{2}^{+}$, denoting the number of anti-self-dual and self-dual two forms of $P$, count the scalar fields arising from the reduction of the 2 -form field living on the 5-brane. For fermions we have $[40,102]$

$$
\begin{aligned}
& N_{L}^{F}=4 h_{1,0}(P), \\
& N_{R}^{F}=4 h_{2,0}(P)+4 .
\end{aligned}
$$

Under the assumption that the Calabi-Yau 3-fold $M$ does not have 1-cycle and that the 4-cycle $P$ is ample, the authors of [102] gave a formula for $d_{p}(P)$ and used it to compute the number of left- and right-moving fermions and bosons. We however have a Calabi-Yau manifold with two 1-cycles $\widehat{S}^{1}$ and $S_{M}^{1}$, and hence the formulæ of [102] are not directly applicable. Thus we need to proceed a little differently following [40]. On a compact Kähler manifold we have the relations:

$$
b_{2} \equiv b_{2}^{+}+b_{2}^{-}=2 h_{2,0}+h_{1,1}, \quad b_{2}^{-}=h_{1,1}-1 .
$$

Substituting this into (6.2) and (6.3) we get

$$
N_{R}^{B}-N_{R}^{F}=d_{p}(P)-2 h_{2,0}(P) .
$$

Now since supersymmetry acts on the right-movers, the number of right-moving bosons and fermions must be equal. This gives

$$
d_{p}(P)=2 h_{2,0}(P) .
$$

This agrees with the result given in [41]. Substituting this into (6.2) and (6.3) we get [41]

$$
N_{L}^{B}=2 h_{2,0}(P)+h_{1,1}(P)+2=b_{\text {even }}(P), \quad N_{L}^{F}=4 h_{1,0}(P)=b_{\text {odd }}(P),
$$

where $b_{\text {even }}(P)$ and $b_{\text {odd }}(P)$ are the dimensions of the even and odd cohomologies of $P$. Thus $c_{L, \text { eff }}^{\text {micro }}$ given in (6.1) is just the Euler character of $P$. This in turn has a simple expression in terms of the 2 -form $P$ representing the 4-cycle $P$ [102]:

$$
c_{L, e f f}^{\text {micro }}=\chi(P)=\int_{M}\left(P \wedge P \wedge P+P \wedge c_{2}(M)\right) .
$$

Evaluating this for the particular brane configuration we have, we get

$$
c_{L, \text { eff }}^{\text {micro }}=6 K\left(Q_{1} Q_{5}+4\right) .
$$


This is in perfect agreement with the formula for the index of the D1-D5-p-KK system given in (5.36), which in turn is in agreement with the macroscopic result given in (3.34). If instead we had calculated the central charge that controls the growth of absolute degeneracy, then we would get the result [40]

$$
c_{L}^{\text {micro }}=N_{L}^{B}+\frac{1}{2} N_{L}^{F}=c_{L, e f f}^{\text {micro }}+\frac{3}{2} N_{L}^{F}=6\left(K Q_{1} Q_{5}+4 K+1\right),
$$

since $N_{L}^{F}=4 h_{1,0}(P)=4 h_{1,0}(M)=4$. As noted in [39, 40], (6.10) fails to agree with the macroscopic result (3.34). Thus we see that the apparent puzzle in [39, 40] arose from comparing the microscopic degeneracy with the macroscopic index, and there is no disagreement as long as we compare the index on both sides.

\subsection{M5-brane on $T^{7}$}

We shall now repeat the analysis of section 6.1 with $\mathrm{K} 3$ replaced by $T^{4}$, i.e. directly compute the microscopic index of the system of M5-branes wrapped on $T^{7}$ without mapping it to the D1-D5-p-KK monopole system. Let us label the $T^{7}$ by coordinates 1-7. In this theory we consider a configuration with $Q_{1}$ M5-branes wrapped along 12345 directions, $Q_{5}$ M5-branes wrapped along 12367 directions and $K$ M5-branes wrapped along 14567 directions, carrying momentum $n$ along the 1-direction. This configuration breaks 28 out of 32 supersymmetries of the theory and hence the relevant helicity trace index is $B_{14}$. Following the analysis of section 6.1 we arrive at the same result (6.8) for the effective central charge $c_{L, \text { eff }}^{\text {micro }}$. However since $c_{2}$ vanishes on $T^{6}$, we get

$$
c_{L, e f f}^{\text {micro }}=6 Q_{1} Q_{5} K
$$

and hence

$$
\ln d_{\text {micro }}\left(n, Q_{1}, Q_{5}, K\right)=2 \pi \sqrt{Q_{1} Q_{5} K n} .
$$

This agrees with the result (5.46) computed from the D1-D5-p-KK monopole system, in agreement with the duality symmetry. More importantly for us, it agrees with the macroscopic prediction (3.38). If instead of using the effective central charge we had used the actual central charge computed in the limit of free theory, we would get $c_{L}^{\text {micro }}=6\left(Q_{1} Q_{5} K+3\right)$ since we now have $h_{1,0}(P)=h_{1,0}(M)=3$. This would not agree with the macroscopic result.

\section{Why do the microscopic and macroscopic results agree?}

So far we have computed the index of various systems in the macroscopic and the microscopic sides and shown that they agree. However given that on the macroscopic side the index is expressed in terms of the coefficients of the Chern-Simons terms in the action, one might hope that this agreement can be proved in general without having to explicitly compute the index in each case. We shall now show that this is indeed the case. This argument is closely related to the one given in [60], but takes into account the additional subtlety that arises due to the failure of the identification R-symmetry group of the brane 
world-volume theory with the spatial rotation group. For definiteness we shall present the argument for five dimensional black holes; the only change in four dimensions will be that we need to drop all references to the $\mathrm{SU}(2)_{L}$ part of the spatial rotation group and interprete $\mathrm{SU}(2)_{R}$ as the full rotation group.

The argument goes as follows. For black holes of the type considered here, the low energy dynamics of the system of branes underlying the microscopic description of the black hole is described by a $(0,4)$ superconformal field theory. We shall divide the system into two parts. One part which we shall call the regular part has the property that the rightmoving SU(2) R-symmetry current, associated with the $(0,4)$ superconformal symmetry on the world-sheet of the branes, can be identified with the $\mathrm{SU}(2)_{R}$ subgroup of the spatial rotation group. Furthermore the action of the $\mathrm{SU}(2)_{L}$ subgroup of the spatial rotation group on the regular part must correspond to the group generated by the zero modes of a left-moving $\mathrm{SU}(2)$ current algebra on the brane world-sheet theory. The second part does not satisfy this property, and will be called the irregular part. This in particular will contain the center of mass degrees of freedom for which the non-chiral scalars are charged under both $\mathrm{SU}(2)_{L}$ and $\mathrm{SU}(2)_{R}$. Clearly this decomposition is not unique since we can include part of the regular modes into the irregular part, and we can utilise this freedom to choose the irregular part to our convenience. We can now express the total contribution to the index as a combination of the contribution from the two parts as in section 2, treating the regular part in the same way as the modes associated with the bulk of $A d S_{3}$ and the irregular part in the same way as the exterior modes. In particular if we denote by $c_{L, e f f}^{\text {micro }}$ and $k_{L, \text { eff }}^{\text {micro }}$ the quantities which control the growth of the microscopic index, we have the relation analogous to $(2.24)$ :

$$
c_{L, e f f}^{\text {micro }} \equiv c_{L}^{\text {reg }}+c_{L, e f f}^{\text {irreg }}, \quad k_{L, e f f}^{\text {micro }} \equiv k_{L}^{\text {reg }}+k_{L, e f f}^{\text {irreg }} .
$$

As in section 2, we shall denote by $k_{L}, k_{R}$ and $c_{\text {grav }}$ the contribution to $\mathrm{SU}(2)_{R}, \mathrm{SU}(2)_{L}$ and gravitational anomaly from various fields on the brane world-volume. In (7.1) we have used the fact that for the regular part the identification of the R-symmetry group with the spatial rotation group allows us to conclude, as in the case of the bulk modes, that the quantities which control the growth of the index are the same as the ones which control the growth of degeneracy, that is the central charge $c_{L}^{r e g}$ of the left-moving Virasoro algebra and the anomaly $k_{L}^{r e g}$ of $\mathrm{SU}(2)_{L} \cdot{ }^{18}$ Furthermore we also have the relations:

$$
c_{\text {grav }}^{r e g}=c_{L}^{r e g}-c_{R}^{r e g}, \quad c_{R}^{r e g}=6 k_{R}^{r e g} .
$$

Let us denote by $k_{L}^{\text {micro }}, k_{R}^{\text {micro }}$ and $c_{g r a v}^{\text {micro }}$ the total contribution to the $\mathrm{SU}(2)_{L}, \mathrm{SU}(2)_{R}$ and the gravitational anomaly from all the microscopic degrees of freedom. Then we have the relations:

$$
k_{L}^{\text {micro }}=k_{L}^{\text {reg }}+k_{L}^{\text {irreg }}, \quad k_{R}^{\text {micro }}=k_{R}^{\text {reg }}+k_{R}^{\text {irreg }}, \quad c_{\text {grav }}^{\text {micro }}=c_{\text {grav }}^{\text {reg }}+c_{\text {grav }}^{\text {irreg }} .
$$

\footnotetext{
${ }^{18} \mathrm{An}$ indirect evidence for the presence of the irregular part follows from the observations of section 5 , section 6 that in the microscopic theory the index and degeneracies do not always agree. Since for the regular part the index and the degeneracy grow in the same manner, the difference can be attributed to the presence of the irregular part. Later we shall explicitly see examples of irregular parts of the microscopic system.
} 
Using (7.1)-(7.3) we get

$$
c_{L, e f f}^{\text {micro }}=c_{\text {grav }}^{\text {micro }}+6 k_{R}^{\text {micro }}+\Delta_{\text {micro }}, \quad k_{L, e f f}^{\text {micro }}=k_{L}^{\text {micro }}+\delta_{\text {micro }},
$$

where

$$
\begin{aligned}
\Delta_{\text {micro }} \equiv-6 k_{R}^{\text {irreg }}-c_{\text {grav }}^{\text {irreg }}+c_{L, \text { eff }}^{\text {irreg }} & =-6 k_{R}^{\text {irreg }}-\left(c_{L}^{\text {irreg }}-c_{R}^{\text {irreg }}\right)+c_{L, \text { eff }}^{\text {irreg }}, \\
\delta_{\text {micro }} & =k_{L, \text { eff }}^{\text {irreg }}-k_{L}^{\text {irreg }} .
\end{aligned}
$$

These are the analogs of eqs.(4.10) and (4.11) in the macroscopic theory. We can now proceed in the same way as in section 4 to show that $\Delta_{\text {micro }}$ and $\delta_{\text {micro }}$ vanish. For this we need to make the same assumptions on the structure of the irregular modes as we had to do on the structure of the exterior modes in section 4 . Thus we get

$$
c_{L, e f f}^{\text {micro }}=c_{\text {grav }}^{\text {micro }}+6 k_{R}^{\text {micro }}, \quad k_{L, \text { eff }}^{\text {micro }}=k_{L}^{\text {micro }} .
$$

Finally we make use of the observation that the coefficients of the gauge and Lorentz ChernSimons terms in the bulk theory are related to the gauge and gravitational anomalies on this brane configuration $[60,103]$. This allows us to conclude that $c_{\text {grav }}^{\text {micro }}, k_{R}^{\text {micro }}$ and $k_{L}^{\text {micro }}$ must be equal to $c_{\text {grav }}^{\text {asymp }}, k_{R}^{\text {asymp }}$ and $k_{L}^{a s y m p}$ - the coefficients of the Lorentz, $\mathrm{SU}(2)_{R}$ and $\mathrm{SU}(2)_{L}$ Chern-Simons term in the effective action. Thus from (4.13) we get

$$
c_{L, \text { eff }}^{\text {micro }}=c_{L, e f f}^{\text {macro }}, \quad k_{L, \text { eff }}^{\text {micro }}=k_{L, \text { eff }}^{\text {macro }} .
$$

This establishes the equivalence of the macroscopic and the microscopic index.

We shall now explicitly compute the coefficients $c_{\text {grav }}^{\text {micro }}, k_{R}^{\text {micro }}$ and $k_{L}^{\text {micro }}$ in some examples by computing the anomalies due to the world-volume fields and show that the results agree with the explicit microscopic results for the index given in section 5 and section 6 . During this analysis we shall also identify the irregular modes in various systems. We begin with the D1-D5-p system on $K 3 \times S^{1}$ in the type IIB Cardy limit, For simplicity we shall take $Q_{5}=1$. Since a D5-brane wrapped on K3 carries -1 unit of D1-brane charge, we need $\left(Q_{1}+1\right)$ D1-branes to produce $Q_{1}$ units of D1-brane charge. In this case the world-volume bosonic degrees of freedom consist of $4\left(Q_{1}+1\right)$ scalars describing D1-brane motion along $K 3$ and 4 scalars describing the overall motion of the D1-D5-brane system in the transverse direction. The former are all neutral under the $\mathrm{SU}(2)_{L} \times \mathrm{SU}(2)_{R}$ rotation group in the space transverse to the D1-D5-brane world-volume, while the latter are in the $\left(2_{L}, 2_{R}\right)$ representation of $\mathrm{SU}(2)_{L} \times \mathrm{SU}(2)_{R}$. Since these scalars are non-chiral they do not contribute to $\mathrm{SU}(2)_{L} \times \mathrm{SU}(2)_{R}$ anomaly. In order to determine the $\mathrm{SU}(2)_{L} \times \mathrm{SU}(2)_{R}$ quantum numbers of the fermions we can use the $(4,4)$ supersymmetry of the world-volume theory. Since the left/right moving modes are paired by supercharges which are doublets of $\mathrm{SU}(2)_{L} / \mathrm{SU}(2)_{R}$, the fermionic partners of the $4\left(Q_{1}+1\right)$ neutral scalars consist of a total of $4\left(Q_{1}+1\right)$ left-moving fermions in the representation $\left(2_{L}, 1_{R}\right)$ and $4\left(Q_{1}+1\right)$ right-moving fermions in the representation $\left(1_{L}, 2_{R}\right)$. On the other hand the fermionic partners of the $\left(2_{L}, 2_{R}\right)$ scalars representing the transverse motion will consist of 4 left-moving fermions in the representation $\left(1_{L}, 2_{R}\right)$ and 4 right-moving fermions in the representation $\left(2_{L}, 1_{R}\right)$. 
Thus as far as the $\mathrm{SU}(2)_{L}$ group is concerned, we have altogether $4\left(Q_{1}+1\right)$ left-moving fermions and 4 right-moving fermions belonging to the doublet representation of $\mathrm{SU}(2)_{L}$. This gives a total contribution of $Q_{1}+1-1=Q_{1}$ to the $\mathrm{SU}(2)_{L}$ anomaly coefficient $k_{L}^{\text {micro }}$. A similar counting gives $k_{R}^{\text {micro }}=Q_{1}$. On the other hand since the spectrum on the brane is left-right symmetric, the gravitational anomaly $c_{\text {grav }}^{\text {micro }}$ vanishes. Eq. (7.7) now gives $c_{L, e f f}^{\text {micro }}=6 Q_{1}$ and $k_{L, e f f}^{\text {micro }}=Q_{1}$. This is in agreement with the microscopic result (5.19).

This analysis also throws some light on the origin of the discrepancy between $c_{L, e f f}^{\text {micro }}=$ $6 Q_{1}$ - the quantity that controls the growth of the index on the microscopic side, and $c_{L}^{\text {micro }}=\left(Q_{1}+2\right)-$ the quantity that controls the growth of the microscopic degeneracy at weak coupling. As argued before, for regular part $c_{L}=c_{L, e f f}$; so the difference must be due to the irregular part. In this case the irregular part comes from the $\left(2_{L}, 2_{R}\right)$ scalars representing the transverse motion of the brane and their fermionic partners. As argued above these include 4 left-moving fermions in the representation $\left(1_{L}, 2_{R}\right)$ and 4 right-moving fermions in the representation $\left(2_{L}, 1_{R}\right)$. Now the SU(2) R-symmetry current on the brane world-volume, associated with the $(0,4)$ superconformal algebra, is right-moving. Hence all the left-moving fermions and bosons must be neutral under it. In contrast we see that the left-moving components of the $\left(2_{L}, 2_{R}\right)$ scalars and the left-moving $\left(1_{L}, 2_{R}\right)$ fermions are in the doublet representation of the $\mathrm{SU}(2)_{R}$ spatial rotation. Thus on these fields the $\mathrm{SU}(2)$ R-symmetry action cannot be identified as the action of the $\mathrm{SU}(2)_{R}$ spatial rotation, and they must be considered as part of the irregular modes. Indeed by carefully examining the computation of $c_{L, \text { eff }}^{\text {micro }}$ given above one can easily see that it is due to the presence of these irregular modes that $c_{L, \text { eff }}^{\text {micro }}$ and $c_{L}^{\text {micro }}$ differ. Similarly for regular modes we also require that the spatial $\mathrm{SU}(2)_{L}$ rotation acts as the zero mode of a left-moving $\mathrm{SU}(2)$ current algebra. Thus all the right-moving regular modes must be neutral under $\mathrm{SU}(2)_{L}$. This fails for the right-moving $\left(2_{L}, 2_{R}\right)$ scalars and $\left(2_{L}, 1_{R}\right)$ fermions, showing that they must also be part of the irregular modes.

The explicit computation of $c_{L}^{\text {micro }}$ and $k_{L}^{\text {micro }}$ for the D1-D5-p system on $T^{4} \times S^{1}$ in the type IIB Cardy limit is almost identical. In this case the D5-brane on $T^{4}$ does not carry any D1-brane charge and we have $4 Q_{1}$ bosons associated with the motion of the D1-brane inside the D5-brane and 4 extra bosons associated with Wilson line on the D5-brane along $T^{4}$. All of these are neutral under $\mathrm{SU}(2)_{L} \times \mathrm{SU}(2)_{R}$. We also have four transverse bosons in the $\left(2_{L}, 2_{R}\right)$ representation of the $\mathrm{SU}(2)_{L} \times \mathrm{SU}(2)_{R}$. Thus the total spectrum of bosons is identical to that in the case of D1-D5-p system on $K 3 \times S^{1}$, and due to supersymmetry the fermionic spectrum is also identical. Thus we still have $k_{R}^{\text {micro }}=Q_{1}, k_{L}^{\text {micro }}=Q_{1}$, $c_{\text {grav }}^{\text {micro }}=0$, and eq.(7.7) leads to $c_{L, e f f}^{\text {macro }}=6 Q_{1}$, in agreement with the microscopic result for the index given in (5.42).

For the D1-D5-p system in the type IIA Cardy limit the underlying microscopic system is the system of $Q_{5}$ NS5-branes and $Q_{1}$ fundamental strings. The dynamics of this system is not well understood and hence we do not have an independent calculation of $c_{\text {grav }}^{\text {micro }}, k_{R}^{\text {micro }}$ and $k_{L}^{\text {micro }}$ from the computation of anomalies in the microscopic theory. Nevertheless the macroscopic results for these quantities, as well as the exact results for the microscopic index derived in the dual type IIB frame, tells us what these anomaly coefficients should be.

A similar analysis can be carried out for the MSW string [40] analyzed in section 6 . We consider M-theory on $M \times S^{1}$ where $M$ can be either $K 3 \times T^{2}$ or $T^{6}$ and take an M5-brane 
wrapped on a four cycle $P$ in $M$ times $S^{1}$. According to eqs.(6.2)-(6.7) the number of leftand right-moving bosons and fermions are given by:

$$
\begin{aligned}
& N_{L}^{B}=2 h_{2,0}(P)+h_{1,1}(P)+2, \\
& N_{L}^{F}=4 h_{1,0}(P), \\
& N_{R}^{B}=4 h_{2,0}(P)+4 \text {, } \\
& N_{R}^{F}=4 h_{2,0}(P)+4 \text {. }
\end{aligned}
$$

This gives the gravitational anomaly coefficient in the microscopic theory to be

$$
c_{\text {grav }}^{\text {micro }}=N_{L}^{B}+\frac{1}{2} N_{L}^{F}-N_{R}^{B}-\frac{1}{2} N_{R}^{F}=h_{1,1}(P)-4 h_{2,0}(P)+2 h_{1,0}(P)-4 .
$$

Next we turn to the computation of $k_{R}^{\text {micro }}$, - the anomaly in the spatial rotation symmetry. ${ }^{19}$ The chiral bosons associated with the component of the 2 -form field along the M5-brane world-volume are neutral under SU(2) and hence cannot contribute to the $\mathrm{SU}(2)$ anomaly. The non-chiral bosons of course also do not contribute to the SU(2) anomaly. The $N_{R}^{F}$ right-moving fermions are doublets of $\mathrm{SU}(2)$ and give a contribution of $N_{R}^{F} / 4$ to $k_{R}^{\text {micro }}$ whereas the $N_{L}^{F}$ left-moving fermions are also doublets of SU(2) and give a contribution of $-N_{L}^{F} / 4$. Thus the net contribution to $k_{R}^{m i c r o}$ is given by

$$
k_{R}^{\text {micro }}=\frac{1}{4}\left(N_{R}^{F}-N_{L}^{F}\right)=h_{2,0}(P)-h_{1,0}(P)+1 .
$$

Using (7.7), (7.10) and (7.11) we get

$$
c_{L, e f f}^{\text {micro }}=c_{\text {grav }}^{\text {micro }}+6 k_{R}^{\text {micro }}=h_{1,1}(P)+2 h_{2,0}(P)-4 h_{1,0}(P)+2=\chi(P) .
$$

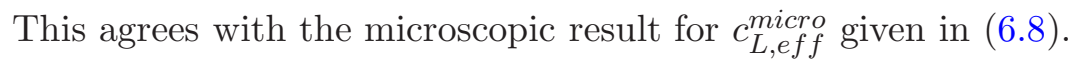

Note that (7.12) does not agree with the microscopic central charge

$$
c_{L}^{\text {micro }}=N_{L}^{B}+\frac{1}{2} N_{L}^{F}=h_{1,1}(P)+2 h_{2,0}(P)+2 h_{1,0}(P)+2 .
$$

Again the difference can be traced to the contribution from the irregular modes. For example there are $4 h_{1,0}(P)$ left-moving fermions which transform as doublets of the spatial $\mathrm{SU}(2)$ rotation group. Since the left-moving fermions must be neutral under the rightmoving R-symmetry current, on these fermions the R-symmetry and spatial rotation act differently. Thus they must be considered as part of the irregular modes.

\section{Acknowledgments}

It is a pleasure to thank Nabamita Banerjee, Justin David, Bernard de Wit, Rajesh Gopakumar, Per Kraus, Shiraz Minwalla and Jan Troost for valuable discussions. The work of A. D. was supported in part by the Excellence Chair of the Agence Nationale de la Recherche (ANR). The work of J. G was supported in part by Fundacão para Ciência e Tecnologia (FCT). The work of S. M. is supported in part by the European Commission Marie Curie Fellowship under the contract PIIF- GA-2008-220899. The work of A. S. was supported in part by the J. C. Bose fellowship of the Department of Science and Technology, India and by the Chaires Internationales de Recherche Blaise Pascal, France.

\footnotetext{
${ }^{19}$ Note that in this case there is no $\mathrm{SU}(2)_{L}$ symmetry since we are considering a black hole in $3+1$ dimensions.
} 


\section{A Chern-Simons contribution from higher derivative terms}

In this section we describe, following [88], how to compute the gauge and Lorentz ChernSimons terms in $A d S_{3}$ by starting with a six dimensional action and dimensionally reducing it on $A d S_{3} \times S^{3}$. The six dimensional theory will be assumed to have metric and a 2form field $B$ as the fundamental fields, but inclusion of other fields in the discussion is straightforward. We shall denote by $H=d B$ the 3 -form field strength. First consider a theory with manifestly gauge and general coordinate invariant Lagrangian density given as a function of $H, g_{\mu \nu}$, the Riemann tensor and covariant derivatives of these fields. Dimensional reduction of the metric on $S^{3}$ produces $\mathrm{SO}(4)$ gauge fields. When all the fluctuating fields around the $A d S_{3} \times S^{3}$ background, including these $\mathrm{SO}(4)$ gauge fields, are set to zero then the background 3-form field on $A d S_{3} \times S^{3}$ takes the form:

$$
H_{3}=\frac{a}{4} \epsilon_{3}+b * \epsilon_{3},
$$

where $\epsilon_{3}$ is the unit 3 -sphere volume form, normalized so that $\int_{S^{3}} \epsilon_{3}=16 \pi^{2}$, $*$ denotes Hodge dual in six dimensions and $a$ and $b$ are two constants. We shall normalize the 2form field so that $\int H_{3}$ is quantized in integer units. The quantized electric and magnetic charges $Q$ and $P$ associated with this background are now defined through the equations:

$$
\int_{S^{3}} H_{3}=4 \pi^{2} P
$$

and ${ }^{20}$

$$
\int_{S^{3}}\left(\frac{\delta S_{0}}{\delta H_{3}}\right)=\frac{Q}{2 \pi}
$$

where $S_{0}$ is the action obtained by integrating the gauge and diffeomorphism invariant lagrangian density over $A d S_{3} \times S^{3}$. Eq. (A.1) gives

$$
a=P \text {. }
$$

$b$ is related to $Q$ but this relation depends on the form of the action $S$.

Let us now consider the effect of switching on the fields describing fluctuations around the $A d S_{3} \times S^{3}$ background. Dimensional reduction of the metric on $S^{3}$ produces a set of $\mathrm{SO}(4)=\mathrm{SU}(2)_{L} \times \mathrm{SU}(2)_{R}$ gauge fields $A_{L}, A_{R}$ on $A d S_{3}$. When these gauge fields are non-zero we need to replace (A.1) by [88]

$$
H_{3}=4 \pi^{2} a\left(e_{3}(A)-\chi_{3}(A)\right)+b * \epsilon_{3} .
$$

Here $e_{3}(A)$ is 3 -form on $A d S_{3} \times S^{3}$ defined in [88] and has the property that $\int_{S^{3}} e_{3}=1$ and that when the $\mathrm{SO}(4)$ gauge fields are set to zero $e_{3}$ reduces to $\epsilon_{3} / 16 \pi^{2}$. $\chi_{3}$ is the Chern-Simons term for the $\mathrm{SO}(4)$ gauge fields:

$$
\begin{aligned}
\chi_{3} & =\frac{1}{8 \pi^{2}}\left(\omega\left(A_{R}\right)-\omega\left(A_{L}\right)\right), \\
\omega(A) & \equiv \operatorname{Tr}\left(A \wedge d A+\frac{2}{3} A \wedge A \wedge A\right) .
\end{aligned}
$$

\footnotetext{
${ }^{20}$ While regarding $\delta S_{0} / \delta H_{3}$ as a 3 -form, we need to lower the indices using the $\varepsilon$ tensor as $\left(\delta S_{0} / \delta H_{3}\right)_{\mu \nu \rho}=$ $\left(\delta S_{0} / \delta\left(H_{3}\right)_{\alpha \beta \gamma}\right) \varepsilon_{\alpha \beta \gamma \mu \nu \rho}$.
} 
The trace is taken over the fundamental representation of $\mathrm{SU}(2)$. Note that since $\int_{S^{3}} e_{3}=1$ and $\chi_{3}$ is directed along the $A d S_{3}$ component, the background (A.5) continues to carry magnetic charge $P=a$ defined via (A.2). Now one can show that $e_{3}(A)$ is invariant under $\mathrm{SO}(4)$ gauge transformation [88], but due to the presence of $\chi_{3}$ in (A.5), $H_{3}$ is no longer gauge invariant. Under an $\mathrm{SO}(4)$ gauge transformation denoted by $\delta$, we have

$$
\delta H_{3}=-4 \pi^{2} a d \chi_{2}=-4 \pi^{2} P d \chi_{2},
$$

where $\chi_{2}$ is defined via the equation:

$$
\delta \chi_{3}=d \chi_{2}
$$

The variation of the action under this gauge transformaion is then given by

$$
\delta S_{0}=4 \pi^{2} P \int d \chi_{2} \wedge\left(\frac{\delta S_{0}}{\delta H_{3}}\right)
$$

Now since $d \chi_{2}$ has components only along $A d S_{3}$, we must pick the component of $\left(\frac{\delta S_{0}}{\delta H_{3}}\right)$ along $S^{3}$. Using (A.3) we now get ${ }^{21}$

$$
\delta S_{0}=2 \pi P Q \int_{A d S_{3}} d \chi_{2}
$$

which is the gauge variation of a three dimensional Chern-Simons terms

$$
2 \pi P Q \int_{A d S_{3}} \chi_{3}=\frac{P Q}{4 \pi} \int_{A d S_{3}}\left[-\omega\left(A_{L}\right)+\omega\left(A_{R}\right)\right] .
$$

Using the standard relation between the coefficients of the Chern-Simons terms and the level $\left(k_{R}^{b u l k}, k_{L}^{b u l k}\right)$ of the current algebra in the boundary theory $[60,104,105]$ we get from (A.12)

$$
k_{R}^{b u l k}=k_{L}^{b u l k}=P Q .
$$

For the case of D1-D5 system in type IIB Cardy limit it follows from (3.2), (3.4), (A.2) and (A.3) that we have $P=Q_{5}, Q=Q_{1}$ and hence $P Q=Q_{1} Q_{5}$. In the type IIA Cardy limit the system is an NS5-brane fundamental string system and we have $P=Q_{5}, Q=n$ and hence $P Q=Q_{5} n$.

So far we have assumed that the six dimensional Lagrangian density is gauge and diffeomorphism invariant. Let us now discuss the effect of the Chern-Simons term in the six dimensional action of the form

$$
S_{C S}=-\frac{\beta}{32 \pi^{3}} \int_{A d S_{3} \times S^{3}} H_{3} \wedge \omega_{v}(\Gamma)=\frac{\beta}{32 \pi^{3}} \int_{A d S_{3} \times S^{3}} \omega_{v}(\Gamma) \wedge H_{3},
$$

where $\Gamma$ is the six-dimensional spin connection, and $\omega_{v}(\Gamma)$ is the Lorentz Chern-Simons term

$$
\omega_{v}(\Gamma)=\operatorname{Tr}_{v}\left(\Gamma \wedge d \Gamma+\frac{2}{3} \Gamma \wedge \Gamma \wedge \Gamma\right)
$$

\footnotetext{
${ }^{21}$ We are using the sign convention that $\int_{A d S_{3} \times S^{3}} B_{A d S_{3}} \wedge A_{S^{3}}=\left(\int_{S^{3}} A_{S^{3}}\right)\left(\int_{A d S_{3}} B_{A d S_{3}}\right)$ for 3-forms $A$ and $B$ on $S^{3}$ and $A d S_{3}$ respectively.
} 
the trace being taken over the vector representation of $\mathrm{SO}(6)$. For field configurations of the type we are considering we have

$$
\omega_{v}(\Gamma)=\omega_{v}\left(\Gamma_{A d S_{3}}\right)+\omega_{v}(A)
$$

where $\Gamma_{A d S_{3}}$ denotes the spin connection in $A d S_{3}$ and $A$ denotes the $\mathrm{SO}(4)$ gauge fields associated with the compactification on $S^{3}$. After integrating over $S^{3}$ the Chern-Simons term (A.14) reduces to

$$
\frac{\beta}{8 \pi} P \int_{A d S_{3}}\left[\omega_{v}\left(\Gamma_{A d S_{3}}\right)+\omega_{v}(A)\right]
$$

Now the gauge field $A$ can be decomposed into $\mathrm{SU}(2)_{L}$ and $\mathrm{SU}(2)_{R}$ parts $A_{L}$ and $A_{R}$, and the trace over the vector representation of $\mathrm{SO}(4)$ will give twice the trace over the fundamental representation of $\mathrm{SU}(2)_{L}$ and $\mathrm{SU}(2)_{R}$. This enables us to write (A.17) as

$$
\int_{A d S_{3}}\left[\frac{\beta P}{8 \pi} \omega_{v}\left(\Gamma_{A d S_{3}}\right)+\frac{\beta P}{4 \pi} \omega\left(A_{R}\right)+\frac{\beta P}{4 \pi} \omega\left(A_{L}\right)\right]
$$

where in computing $\omega\left(A_{R, L}\right)=\operatorname{Tr}_{f}\left(A_{L, R} \wedge d A_{L, R}+\frac{2}{3} A_{L, R} \wedge A_{L, R} \wedge A_{L, R}\right)$ we compute the trace in the fundamental representation. Using the standard relation between the Chern-Simons coefficients and the central charges [60, 104, 105] we now get the following one loop corrections to the various central charges:

$$
\Delta c_{\text {grav }}^{\text {bulk }}=12 \beta P, \quad \Delta k_{R}^{\text {bulk }}=\beta P, \quad \Delta k_{L}^{b u l k}=-\beta P .
$$

Finally we shall briefly discuss possible effect of Chern-Simons terms on the definition of the charges. For this we note first that the correct definition of the electric and magnetic charges is via eq.(A.2) and (A.3), but with the $S^{3}$ located at infinity instead of in the intermediate $A d S_{3}$ region. Thus the question is whether the value of the integrals change as we move the integration surface from the intermediate $A d S_{3}$ region to asymptotic infinity. Since $H_{3}=d B$, the integral (A.2) does not change. On the other hand due to the presence of the Chern-Simons term in the action we have from the equation of motion of $B$,

$$
d\left(\frac{\delta S_{0}}{\delta H_{3}}\right) \propto \operatorname{Tr}(R \wedge R)
$$

where $R$ is the six dimensional Riemann tensor. Since the topology of the region bounded by asymptotic infinity and the intermediate $A d S_{3}$ geometry has the form of $\mathbb{R} \times S^{3}$, integral of $\operatorname{Tr}(R \wedge R)$ over this region vanishes. Thus we see that the presence of the Chern-Simons term does not change the definition of the electric charge either. ${ }^{22}$

\footnotetext{
${ }^{22}$ Note that if instead we place the system at the center of Taub-NUT space to get a four dimensional black hole [98], then the near horizon geometry and hence the entropy remains the same, but the charge of the system receives an additional contribution from the Chern-Simons term [68]. This can be seen in two ways; by integrating $\operatorname{Tr}(R \wedge R)$ between the horizon and the asymptotic space, or by dimensionally reducing the action on a circle so that the Chern-Simons term takes a covariant form and the contribution of this term to the charge can be calculated using the entropy function formalism.
} 


\section{B Asymptotic expansion}

In this appendix we shall analyze carefully the behavior of the index associated with the D1-D5-p system in various limits and check that possible corrections to the results derived in section 5 are indeed subleading. Our starting point is the integral representation for the index

$$
d_{\text {micro }}\left(n, Q_{1}, J\right)=(-1)^{J+1} \int_{0}^{1} d \rho_{1} \int_{0}^{1} d \sigma_{1} \int_{0}^{1} d v_{1} e^{-2 \pi i\left(\rho n+\sigma Q_{1}+J v\right)} f(\rho, \sigma, v),
$$

where $(\rho, \sigma, v) \equiv\left(\rho_{1}+i \rho_{2}, \sigma_{1}+i \sigma_{2}, v_{1}+i v_{2}\right)$ are three complex parameters and $f(\rho, \sigma, v)=$ $\left(e^{\pi i v}-e^{-\pi i v}\right)^{4} \eta(\rho)^{24} / \Phi_{10}(\rho, \sigma, v)$ for five dimensional black holes and $1 / \Phi_{10}(\rho, \sigma, v)$ for four dimensional black holes. While carrying out this integral we fix $\left(\rho_{2}, \sigma_{2}, v_{2}\right)$ at

$$
\rho_{2}=\Lambda \frac{Q_{1}}{\sqrt{4 n Q_{1}-J^{2}}}, \quad \sigma_{2}=\Lambda \frac{n}{\sqrt{4 n Q_{1}-J^{2}}}, \quad v_{2}=-\Lambda \frac{J}{2 \sqrt{4 n Q_{1}-J^{2}}},
$$

where $\Lambda$ is a large positive number. For four dimensional black holes this choice gives the degeneracy of single centered black holes [17].

We now consider a family of contours

$$
\rho_{2}=\lambda \frac{Q_{1}}{\sqrt{4 n Q_{1}-J^{2}}}, \quad \sigma_{2}=\lambda \frac{n}{\sqrt{4 n Q_{1}-J^{2}}}, \quad v_{2}=-\lambda \frac{J}{2 \sqrt{4 n Q_{1}-J^{2}}},
$$

where $\lambda$ is a real number. At $\lambda=\Lambda$ we recover the original contour. But we now deform the contour by reducing $\lambda$. As long as the contour does not cross any pole of the integrand the value of the integral remains unchanged. Now the poles of the integrand are given by the divisors of the function $\Phi_{10}(\rho, \sigma, v)$ which are the surfaces

$$
n_{2}\left(\rho \sigma-v^{2}\right)+j v+n_{1} \sigma-m_{1} \rho+m_{2}=0,
$$

where $j$ is any odd integer and the 5 integers $\left(m_{1}, m_{2}, n_{1}, n_{2}, j\right)$ are constrained to satisfy

$$
j^{2}+4\left(m_{1} n_{1}+m_{2} n_{2}\right)-1=0 .
$$

$n_{2}$ can be chosen to be non-negative. The intersection of the codimension 3 subspace given in (B.3) and the codimension 2 subspace given in (B.4) describes a one dimensional curve in the six dimensional space spanned by $(\rho, \sigma, v)$. For fixed $\left(\rho_{2}, \sigma_{2}, v_{2}\right)$ it is an easy exercise to find this curve in the $\left(\rho_{1}, \sigma_{1}, v_{1}\right)$ space and we arrive at the result:

$$
\begin{aligned}
& \rho_{1}=-\frac{n_{1}}{n_{2}}-\frac{1}{\sigma_{2}}\left\{\rho_{2}\left(\sigma_{1}-\frac{m_{1}}{n_{2}}\right)-2 v_{2}\left(v_{1}-\frac{j}{2 n_{2}}\right)\right\} \\
& \frac{\rho_{2}}{\sigma_{2}}\left(\sigma_{1}-\frac{m_{1}}{n_{2}}\right)^{2}+\left(v_{1}-\frac{j}{2 n_{2}}\right)^{2}-2 \frac{v_{2}}{\sigma_{2}}\left(\sigma_{1}-\frac{m_{1}}{n_{2}}\right)\left(v_{1}-\frac{j}{2 n_{2}}\right)=\frac{1}{4 n_{2}^{2}}-\left(\rho_{2} \sigma_{2}-v_{2}^{2}\right) .
\end{aligned}
$$

The last equation describes an ellipse in the $\left(\sigma_{1}, v_{1}\right)$ plane for $\left(\rho_{2} \sigma_{2}-v_{2}^{2}\right)<\left(4 n_{2}^{2}\right)^{-2}$ and has no solution otherwise. Using (B.3) the condition for the absence of a solution to (B.6) reduces to

$$
\lambda>\frac{1}{n_{2}} .
$$


This shows that as long as $\lambda$ is larger then 1 , none of the poles of the integrand intersect the contour and hence the integral will have the same value for all $\lambda>1$. We shall however deform the contour to $\lambda=\frac{1}{2}+\epsilon$ where $\epsilon$ is a small positive number. During the deformation of $\lambda$ from $\Lambda$ to $\frac{1}{2}+\epsilon$ the contour crosses the $n_{2}=1$ poles. The contribution from the residue at this pole was analyzed in section $5 .{ }^{23}$ Our goal will be to analyze the contribution from the contour at $\lambda=\frac{1}{2}+\epsilon$ and argue that this integral is subdominant compared to the residue at the $n_{2}=1$ pole.

Our strategy will be to estimate each term appearing in the integrand separately and then multiply the results to estimate the integrand. First consider the exponential factor in (B.1). For the choice of $\left(\rho_{2}, \sigma_{2}, v_{2}\right)$ given in (B.3) with $\lambda=\frac{1}{2}+\epsilon$, this factor is given by

$$
\exp \left[\left(\frac{1}{2}+\epsilon\right) \pi \sqrt{4 n Q_{1}-J^{2}}\right]
$$

up to a phase.

Next consider the $\left(e^{\pi i v}-e^{-\pi i v}\right)^{4} \eta(\rho)^{24}$ factor that is present in the five dimensional index. Since for (B.3) $\left|e^{\pi i v}-e^{-\pi i v}\right|^{4} \sim 1$ and $|\eta(\rho)|<1$, we can drop this while estimating an upper bound for the integrand. This will allow us to study the corrections to the four and the five dimensional degeneracies together since they differ only due to the presence of the $\eta(\rho)^{24}$ factor. This will also have the advantage that for the five dimensional black holes once we estimate the correction term in the type IIB Cardy limit, we can get the result for the type IIA Cardy limit by exchanging $n$ and $Q_{1}$ since the only term in the integral that breaks this symmetry is the $\eta(\rho)^{24}$ factor.

Finally we turn to an estimate of $1 / \Phi_{10}$. On the subspace (B.3) $\rho_{2} \sigma_{2}-v_{2}^{2}$ is finite, but in the two limits we are interested in, either $\rho_{2}$ or $\sigma_{2}$ becomes small. We do not have a way to find a direct estimate of $\Phi_{10}$ in this region; so we shall use an intuitive reasoning. First of all note that if $\lambda=1 / n_{2}$ then the equations (B.3), (B.6) have a unique solution:

$$
\rho=\frac{i}{2 n_{2} \tau_{2}}-\frac{n_{1}}{n_{2}}, \quad \sigma=i \frac{\tau_{1}^{2}+\tau_{2}^{2}}{2 n_{2} \tau_{2}}+\frac{m_{1}}{n_{2}}, \quad v=\frac{j}{2 n_{2}}-i \frac{\tau_{1}}{2 n_{2} \tau_{2}} .
$$

where

$$
\tau_{1}=\frac{J}{2 Q_{1}}, \quad \tau_{2}=\sqrt{\frac{4 n Q_{1}-J^{2}}{4 Q_{1}^{2}}} .
$$

This represents the unique point on the surface (B.3) with $\lambda=1 / n_{2}$ which also lies on the divisor (B.4). Thus $1 / \Phi_{10}$ diverges there. For $\lambda=\frac{1}{n_{2}}+\epsilon$ the surface (B.3) does not intersect the divisor (B.4), but for sufficiently small $\epsilon$ the two subspaces come close near a point near (B.9). Since $1 / \Phi_{10}$ has a double pole near the divisor (B.4) we expect that as we move along (B.3), $1 /\left|\Phi_{10}\right|$ reaches a local maximum near the point of closest approach to the divisor (B.4), which in turn is close to (B.9). Assuming that the dominant contribution to the integral comes from near this local maximum, we can estimate $1 / \Phi_{10}$ by its behavior near this divisor. This was analyzed in $[53,54]$. We shall here follow the notation of [54]

\footnotetext{
${ }^{23}$ For $n_{2}=1$ we can use the three shift symmetries $\rho \rightarrow \rho+1, \sigma \rightarrow \sigma+1$ and $v \rightarrow v+1$ to set $n_{1}=m_{1}=m_{2}=0$ and $j=1$ [1].
} 
where the analysis was carried out for general value of $n_{2}$. The analysis uses the fact that all the divisors lie in an orbit of $\operatorname{Sp}(2, \mathbb{Z})$ under which the $\Phi_{10}$ is a Siegel modular form of weight 10. At the diagonal divisor $v=0$,

$$
\frac{1}{\Phi_{10}(\rho, \sigma, v)}=-\frac{1}{4 \pi^{2}} \frac{1}{v^{2} \eta^{24}(\rho) \eta^{24}(\sigma)}+\mathcal{O}\left(v^{0}\right) .
$$

One then finds the explicit $\operatorname{Sp}(2, \mathbb{Z})$ transformation which maps the divisor $v=0$ to the generic divisor (B.4), and then uses the modular property of the function $\Phi_{10}$ to find the residue at the generic pole. Thus near such a generic pole we shall have

$$
\frac{1}{\left|\Phi_{10}(\rho, \sigma, v)\right|} \sim \frac{1}{\left|v_{0}^{2} \eta^{24}\left(\rho_{0}\right) \eta^{24}\left(\sigma_{0}\right)\right|} \sim \exp \left[-2 \ln \left|v_{0}\right|-24 \ln \left|\eta\left(\rho_{0}\right) \eta\left(\sigma_{0}\right)\right|\right]
$$

where $\left(\rho_{0}, \sigma_{0}, v_{0}\right)$ are related to $(\rho, \sigma, v)$ by this specific $\operatorname{Sp}(2, \mathbb{Z})$ transformation. In writing (B.12) we have ignored some additional factors related to the modular weight of $\Phi_{10}$, but they do not affect the estimate to leading order. The dominant contribution to the exponent comes from the $-24 \ln \left|\eta\left(\rho_{0}\right) \eta\left(\sigma_{0}\right)\right|$ terms. Thus our goal will be to estimate this term. For sufficiently small $\epsilon$ we can estimate this by evaluating $\rho_{0}$ and $\sigma_{0}$ at the point (B.9). This in turn requires knowing the $\operatorname{Sp}(2, \mathbb{Z})$ transformation that relates $(\rho, \sigma, v)$ to $\left(\rho_{0}, \sigma_{0}, v_{0}\right)$.

Before we proceed we need to define some number theoretic quantities. First, define $r \equiv \operatorname{gcd}\left(n_{1}, n_{2}\right)$, so we can write $r=k_{2} n_{1}-k_{1} n_{2}$ for some $k_{1}, k_{2} \in \mathbb{Z}$. Since (B.5) is satisfied, $r$ must divide $\left(j^{2}-1\right) / 4$. We can then uniquely decompose $r=r_{1} r_{2}$ into a product of relatively prime factors, where $r_{1}$ divides $(j+1) / 2$ and $r_{2}$ divides $(j-1) / 2$. In this convention the result of [54] for $\left(\rho_{0}, \sigma_{0}\right)$ are

$$
\rho_{0}=\delta_{1}+\frac{r_{2}^{2}}{n_{2}}\left(-\tau_{1}+i \tau_{2}\right), \quad \sigma_{0}=\delta_{2}+\frac{r_{1}^{2}}{n_{2}}\left(\tau_{1}+i \tau_{2}\right),
$$

where $\delta_{1}$ and $\delta_{2}$ are constants determined in terms of $m_{i}, n_{i}, j$. In the type IIB Cardy limit we get from (B.10) that $\tau_{2}$ is large. In this limit we get

$$
\left|\eta^{-24}\left(\rho_{0}\right) \eta^{-24}\left(\sigma_{0}\right)\right| \sim \exp \left[\frac{2 \pi}{n_{2}}\left(r_{1}^{2}+r_{2}^{2}\right) \tau_{2}\right] \sim \exp \left[\frac{2 \pi}{n_{2}}\left(r_{1}^{2}+r_{2}^{2}\right) \sqrt{\frac{4 n Q_{1}-J^{2}}{4 Q_{1}^{2}}}\right] .
$$

Let us now focus on the case $n_{2}=2$ since our goal is to estimate the integrand on the contour $\lambda=\frac{1}{2}+\epsilon$. Since $r_{1} r_{2}$ is a divisor of $n_{2}$, for $n_{2}=2$ we have $r_{1}^{2}+r_{2}^{2} \leq 5$. Thus (B.14) gives

$$
\left|\eta^{-24}\left(\rho_{0}\right) \eta^{-24}\left(\sigma_{0}\right)\right|_{\sim}^{<} \exp \left[5 \pi \sqrt{\frac{4 n Q_{1}-J^{2}}{4 Q_{1}^{2}}}\right] .
$$

The result for the type IIA Cardy limit may be obtained by exchanging $Q_{1}$ and $n$ in (B.15):

$$
\left|\eta^{-24}\left(\rho_{0}\right) \eta^{-24}\left(\sigma_{0}\right)\right|_{\sim}^{<} \exp \left[5 \pi \sqrt{\frac{4 n Q_{1}-J^{2}}{4 n^{2}}}\right] .
$$

Combining (B.8) with (B.15), (B.16) we arrive at the following estimates for the correction $\delta d_{\text {micro }}$ to the index $d_{\text {micro }}$ at $\lambda=\frac{1}{2}+\epsilon$. In the type IIB Cardy limit we have

$$
\delta d_{\text {micro }} \sim \exp \left[\left(\frac{1}{2}+\epsilon\right) \pi \sqrt{4 n Q_{1}-J^{2}}+5 \pi \sqrt{\frac{4 n Q_{1}-J^{2}}{4 Q_{1}^{2}}}\right]
$$


and in the type IIA Cardy limit

$$
\delta d_{\text {micro }} \sim \exp \left[\left(\frac{1}{2}+\epsilon\right) \pi \sqrt{4 n Q_{1}-J^{2}}+5 \pi \sqrt{\frac{4 n Q_{1}-J^{2}}{4 n^{2}}}\right] .
$$

Comparing (B.17) with the result given in (5.19) we see that the correction terms are smaller than (5.19) if

$$
\sqrt{Q_{1}-\frac{J^{2}}{4 n}}>\frac{1}{2} \sqrt{Q_{1}-\frac{J^{2}}{4 n}}+\frac{5}{2 Q_{1}} \sqrt{Q_{1}-\frac{J^{2}}{4 n}}
$$

This holds for $Q_{1}>5$. Similarly comparing (B.18) with the result given in (5.26) we see that the correction terms are subdominant in the region:

$$
\sqrt{(n+3)\left(1-\frac{J^{2}}{4(n-1) Q_{1}}\right)}>\frac{1}{2} \sqrt{n-\frac{J^{2}}{4 Q_{1}}}+\frac{5}{2 n} \sqrt{n-\frac{J^{2}}{4 Q_{1}}} .
$$

This can be easily satisfied for example by requiring

$$
n-\frac{J^{2}}{4 Q_{1}} \geq 7 .
$$

Neither of these are the best bounds possible, particularly since we have dropped the $(\eta(\rho))^{24}$ factor from the integrand in estimating the correction term. However this analysis shows the existence of the constants $K_{1}, K_{2}$ appearing in the definition of the type IIB and type IIA Cardy limits beyond which our result for the asymptotic behavior of the microscopic index holds. Finally the leading contribution to the four dimensional index in the $n \rightarrow \infty$ limit, given in (5.33), is always larger than the five dimensional index (5.19) in the type IIB Cardy limit, and hence will dominate over the correction given in (B.17) when $Q_{1}>5$.

Open Access. This article is distributed under the terms of the Creative Commons Attribution Noncommercial License which permits any noncommercial use, distribution, and reproduction in any medium, provided the original author(s) and source are credited.

\section{References}

[1] R. Dijkgraaf, E.P. Verlinde and H.L. Verlinde, Counting dyons in $N=4$ string theory, Nucl. Phys. B 484 (1997) 543 [hep-th/9607026] [SPIRES].

[2] G. Lopes Cardoso, B. de Wit, J. Kappeli and T. Mohaupt, Asymptotic degeneracy of dyonic $N=4$ string states and black hole entropy, JHEP 12 (2004) 075 [hep-th/0412287] [SPIRES].

[3] D. Shih, A. Strominger and X. Yin, Recounting dyons in $N=4$ string theory, JHEP 10 (2006) 087 [hep-th/0505094] [SPIRES].

[4] D. Gaiotto, Re-recounting dyons in $N=4$ string theory, hep-th/0506249 [SPIRES].

[5] D. Shih and X. Yin, Exact black hole degeneracies and the topological string, JHEP 04 (2006) 034 [hep-th/0508174] [SPIRES]. 
[6] D.P. Jatkar and A. Sen, Dyon spectrum in CHL models, JHEP 04 (2006) 018 [hep-th/0510147] [SPIRES].

[7] J.R. David, D.P. Jatkar and A. Sen, Product representation of dyon partition function in CHL models, JHEP 06 (2006) 064 [hep-th/0602254] [SPIRES].

[8] A. Dabholkar and S. Nampuri, Spectrum of dyons and black holes in CHL orbifolds using Borcherds lift, JHEP 11 (2007) 077 [hep-th/0603066] [SPIRES].

[9] J.R. David and A. Sen, CHL dyons and statistical entropy function from D1 - D5 system, JHEP 11 (2006) 072 [hep-th/0605210] [SPIRES].

[10] J.R. David, D.P. Jatkar and A. Sen, Dyon spectrum in $N=4$ supersymmetric type-II string theories, JHEP 11 (2006) 073 [hep-th/0607155] [SPIRES].

[11] J.R. David, D.P. Jatkar and A. Sen, Dyon spectrum in generic $N=4$ supersymmetric $\mathbb{Z}_{N}$ orbifolds, JHEP 01 (2007) 016 [hep-th/0609109] [SPIRES].

[12] A. Dabholkar and D. Gaiotto, Spectrum of CHL dyons from genus-two partition function, JHEP 12 (2007) 087 [hep-th/0612011] [SPIRES].

[13] A. Sen, Walls of marginal stability and dyon spectrum in $N=4$ supersymmetric string theories, JHEP 05 (2007) 039 [hep-th/0702141] [SPIRES].

[14] A. Dabholkar, D. Gaiotto and S. Nampuri, Comments on the spectrum of CHL dyons, JHEP 01 (2008) 023 [hep-th/0702150] [SPIRES].

[15] N. Banerjee, D.P. Jatkar and A. Sen, Adding charges to $N=4$ dyons, JHEP 07 (2007) 024 [arXiv:0705.1433] [SPIRES].

[16] A. Sen, Two centered black holes and $N=4$ dyon spectrum, JHEP 09 (2007) 045 [arXiv:0705.3874] [SPIRES].

[17] M.C.N. Cheng and E. Verlinde, Dying dyons don't count, JHEP 09 (2007) 070 [arXiv:0706.2363] [SPIRES].

[18] A. Sen, Black hole entropy function, attractors and precision counting of microstates, Gen. Rel. Grav. 40 (2008) 2249 [arXiv:0708.1270] [SPIRES].

[19] S. Banerjee, A. Sen and Y.K. Srivastava, Generalities of quarter BPS dyon partition function and dyons of torsion two, JHEP 05 (2008) 101 [arXiv:0802.0544] [SPIRES].

[20] S. Banerjee, A. Sen and Y.K. Srivastava, Partition functions of torsion $>1$ dyons in heterotic string theory on $T^{6}$, JHEP 05 (2008) 098 [arXiv: 0802.1556] [SPIRES].

[21] A. Dabholkar, J. Gomes and S. Murthy, Counting all dyons in $N=4$ string theory, arXiv:0803.2692 [SPIRES].

[22] M.C.N. Cheng and E.P. Verlinde, Wall crossing, discrete attractor flow and Borcherds algebra, SIGMA 4 (2008) 068 [arXiv:0806.2337] [SPIRES].

[23] S. Govindarajan and K. Gopala Krishna, Generalized Kac-Moody algebras from CHL dyons, JHEP 04 (2009) 032 [arXiv:0807.4451] [SPIRES].

[24] M.C.N. Cheng and A. Dabholkar, Borcherds-Kac-Moody symmetry of $N=4$ dyons, Commun. Num. Theor. Phys. 3 (2009) 59 [arXiv:0809.4258] [SPIRES].

[25] S. Banerjee, A. Sen and Y.K. Srivastava, Genus two surface and quarter BPS dyons: The contour prescription, JHEP 03 (2009) 151 [arXiv:0808.1746] [SPIRES]. 
[26] M.C.N. Cheng and L. Hollands, A geometric derivation of the dyon wall-crossing group, JHEP 04 (2009) 067 [arXiv:0901.1758] [SPIRES].

[27] S. Govindarajan and K. Gopala Krishna, BKM Lie superalgebras from dyon spectra in $Z_{N}$ CHL orbifolds for composite N, JHEP 05 (2010) 014 [arXiv:0907.1410] [SPIRES].

[28] A. Dabholkar and J. Gomes, Perturbative tests of non-perturbative counting, JHEP 03 (2010) 128 [arXiv:0911.0586] [SPIRES].

[29] A. Sen, A twist in the dyon partition function, JHEP 05 (2010) 028 [arXiv:0911.1563] [SPIRES].

[30] R. Dijkgraaf, G.W. Moore, E.P. Verlinde and H.L. Verlinde, Elliptic genera of symmetric products and second quantized strings, Commun. Math. Phys. 185 (1997) 197 [hep-th/9608096] [SPIRES].

[31] J.M. Maldacena, G.W. Moore and A. Strominger, Counting BPS black holes in toroidal type-II string theory, hep-th/9903163 [SPIRES].

[32] D. Shih, A. Strominger and X. Yin, Counting dyons in $N=8$ string theory, JHEP 06 (2006) 037 [hep-th/0506151] [SPIRES].

[33] B. Pioline, BPS black hole degeneracies and minimal automorphic representations, JHEP 08 (2005) 071 [hep-th/0506228] [SPIRES].

[34] A. Sen, $N=8$ dyon partition function and walls of marginal stability, JHEP 07 (2008) 118 [arXiv: 0803.1014] [SPIRES].

[35] A. Sen, U-duality invariant dyon spectrum in type-II on $T^{6}$, JHEP 08 (2008) 037 [arXiv:0804.0651] [SPIRES].

[36] R.M. Wald, Black hole entropy is the Noether charge, Phys. Rev. D 48 (1993) 3427 [gr-qc/9307038] [SPIRES].

[37] A. Sen, Quantum entropy function from $A d S_{2} / C F T_{1}$ correspondence, Int. J. Mod. Phys. A 24 (2009) 4225 [arXiv:0809.3304] [SPIRES].

[38] A. Sen, Arithmetic of quantum entropy function, JHEP 08 (2009) 068 [arXiv:0903.1477] [SPIRES].

[39] G. Lopes Cardoso, B. de Wit and T. Mohaupt, Macroscopic entropy formulae and non-holomorphic corrections for supersymmetric black holes, Nucl. Phys. B 567 (2000) 87 [hep-th/9906094] [SPIRES].

[40] N. Lambert, The M5-brane on $K 3 \times T^{2}$, JHEP 02 (2008) 060 [arXiv:0712.3166] [SPIRES].

[41] C. Vafa, Black holes and Calabi-Yau threefolds, Adv. Theor. Math. Phys. 2 (1998) 207 [hep-th/9711067] [SPIRES].

[42] C. Bachas and E. Kiritsis, $F^{4}$ terms in $N=4$ string vacua, Nucl. Phys. Proc. Suppl. 55B (1997) 194 [hep-th/9611205] [SPIRES].

[43] A. Gregori et al., $R^{2}$ corrections and non-perturbative dualities of $N=4$ string ground states, Nucl. Phys. B 510 (1998) 423 [hep-th/9708062] [SPIRES].

[44] E. Kiritsis, Introduction to non-perturbative string theory, hep-th/9708130 [SPIRES].

[45] F. Denef and G.W. Moore, Split states, entropy enigmas, holes and halos, hep-th/0702146 [SPIRES]. 
[46] J.B. Gutowski and H.S. Reall, Supersymmetric AdS $S_{5}$ black holes, JHEP 02 (2004) 006 [hep-th/0401042] [SPIRES].

[47] J.B. Gutowski and H.S. Reall, General supersymmetric AdS $S_{5}$ black holes, JHEP 04 (2004) 048 [hep-th/0401129] [SPIRES].

[48] Z.W. Chong, M. Cvetič, H. Lü and C.N. Pope, General non-extremal rotating black holes in minimal five-dimensional gauged supergravity, Phys. Rev. Lett. 95 (2005) 161301 [hep-th/0506029] [SPIRES].

[49] J. Kinney, J.M. Maldacena, S. Minwalla and S. Raju, An index for 4 dimensional super conformal theories, Commun. Math. Phys. 275 (2007) 209 [hep-th/0510251] [SPIRES].

[50] M. Berkooz and D. Reichmann, Weakly renormalized near 1/16 SUSY Fermi liquid operators in $N=4$ SYM, JHEP 10 (2008) 084 [arXiv:0807.0559] [SPIRES].

[51] N. Banerjee, I. Mandal and A. Sen, Black hole hair removal, JHEP 07 (2009) 091 [arXiv:0901.0359] [SPIRES].

[52] D.P. Jatkar, A. Sen and Y.K. Srivastava, Black hole hair removal: non-linear analysis, JHEP 02 (2010) 038 [arXiv:0907.0593] [SPIRES].

[53] N. Banerjee, D.P. Jatkar and A. Sen, Asymptotic expansion of the $N=4$ dyon degeneracy, JHEP 05 (2009) 121 [arXiv:0810.3472] [SPIRES].

[54] S. Murthy and B. Pioline, A Farey tale for $N=4$ dyons, JHEP 09 (2009) 022 [arXiv:0904.4253] [SPIRES].

[55] A. Sen, Arithmetic of $N=8$ black holes, JHEP 02 (2010) 090 [arXiv:0908.0039] [SPIRES].

[56] A. Sen, Discrete information from CHL black holes, JHEP 11 (2010) 138 [arXiv: 1002.3857] [SPIRES].

[57] A. Sen, How do black holes predict the sign of the Fourier coefficients of Siegel modular forms?, arXiv: 1008.4209 [SPIRES].

[58] A. Strominger, Black hole entropy from near-horizon microstates, JHEP 02 (1998) 009 [hep-th/9712251] [SPIRES].

[59] V. Balasubramanian and F. Larsen, Near horizon geometry and black holes in four dimensions, Nucl. Phys. B 528 (1998) 229 [hep-th/9802198] [SPIRES].

[60] P. Kraus and F. Larsen, Microscopic black hole entropy in theories with higher derivatives, JHEP 09 (2005) 034 [hep-th/0506176] [SPIRES].

[61] J. de Boer, F. Denef, S. El-Showk, I. Messamah and D. Van den Bleeken, Black hole bound states in $A d S_{3} \times S^{2}, J H E P 11$ (2008) 050 [arXiv:0802.2257] [SPIRES].

[62] H. Saida and J. Soda, Statistical entropy of BTZ black hole in higher curvature gravity, Phys. Lett. B 471 (2000) 358 [gr-qc/9909061] [SPIRES].

[63] B. Sahoo and A. Sen, BTZ black hole with Chern-Simons and higher derivative terms, JHEP 07 (2006) 008 [hep-th/0601228] [SPIRES].

[64] E. Witten, Anti-de Sitter space and holography, Adv. Theor. Math. Phys. 2 (1998) 253 [hep-th/9802150] [SPIRES].

[65] O. Aharony and E. Witten, Anti-de Sitter space and the center of the gauge group, JHEP 11 (1998) 018 [hep-th/9807205] [SPIRES]. 
[66] A. Bilal and C.-S. Chu, Testing the AdS/CFT correspondence beyond large- $N$, hep-th/0003129 [SPIRES].

[67] J.M. Maldacena, G.W. Moore and N. Seiberg, D-brane charges in five-brane backgrounds, JHEP 10 (2001) 005 [hep-th/0108152] [SPIRES].

[68] A. Castro and S. Murthy, Corrections to the statistical entropy of five dimensional black holes, JHEP 06 (2009) 024 [arXiv: 0807.0237] [SPIRES].

[69] N. Banerjee, Subleading correction to statistical entropy for BMPV black hole, Phys. Rev. D 79 (2009) 081501 [arXiv:0807.1314] [SPIRES].

[70] A. Castro, J.L. Davis, P. Kraus and F. Larsen, Precision entropy of spinning black holes, JHEP 09 (2007) 003 [arXiv:0705.1847] [SPIRES].

[71] A. Castro, J.L. Davis, P. Kraus and F. Larsen, String theory effects on five-dimensional black hole physics, Int. J. Mod. Phys. A 23 (2008) 613 [arXiv:0801.1863] [SPIRES].

[72] B. de Wit and S. Katmadas, Near-horizon analysis of D $=5$ BPS black holes and rings, JHEP 02 (2010) 056 [arXiv:0910.4907] [SPIRES].

[73] E. Witten, On the conformal field theory of the Higgs branch, JHEP 07 (1997) 003 [hep-th/9707093] [SPIRES].

[74] A. Sen, Two charge system revisited: small black holes or horizonless solutions?, JHEP 05 (2010) 097 [arXiv: 0908.3402] [SPIRES].

[75] J.C. Breckenridge, R.C. Myers, A.W. Peet and C. Vafa, D-branes and spinning black holes, Phys. Lett. B 391 (1997) 93 [hep-th/9602065] [SPIRES].

[76] A. Castro, D. Grumiller, F. Larsen and R. McNees, Holographic description of AdS $S_{2}$ black holes, JHEP 11 (2008) 052 [arXiv:0809.4264] [SPIRES].

[77] H.J. Boonstra, B. Peeters and K. Skenderis, Brane intersections, anti-de Sitter spacetimes and dual superconformal theories, Nucl. Phys. B 533 (1998) 127 [hep-th/9803231] [SPIRES].

[78] A. Strominger, AdS 2 quantum gravity and string theory, JHEP 01 (1999) 007 [hep-th/9809027] [SPIRES].

[79] E. Witten, Baryons and branes in anti de Sitter space, JHEP 07 (1998) 006 [hep-th/9805112] [SPIRES].

[80] J. de Boer, M.C.N. Cheng, R. Dijkgraaf, J. Manschot and E. Verlinde, A farey tail for attractor black holes, JHEP 11 (2006) 024 [hep-th/0608059] [SPIRES].

[81] G. Lopes Cardoso, B. de Wit and T. Mohaupt, Corrections to macroscopic supersymmetric black-hole entropy, Phys. Lett. B 451 (1999) 309 [hep-th/9812082] [SPIRES].

[82] T. Mohaupt, Black hole entropy, special geometry and strings, Fortsch. Phys. 49 (2001) 3 [hep-th/0007195] [SPIRES].

[83] A. Castro, J.L. Davis, P. Kraus and F. Larsen, 5D black holes and strings with higher derivatives, JHEP 06 (2007) 007 [hep-th/0703087] [SPIRES].

[84] A. Strominger and C. Vafa, Microscopic origin of the Bekenstein-Hawking entropy, Phys. Lett. B 379 (1996) 99 [hep-th/9601029] [SPIRES].

[85] A. Dabholkar, A. Sen and S.P. Trivedi, Black hole microstates and attractor without supersymmetry, JHEP 01 (2007) 096 [hep-th/0611143] [SPIRES]. 
[86] M. Cvetič and F. Larsen, Near horizon geometry of rotating black holes in five dimensions, Nucl. Phys. B 531 (1998) 239 [hep-th/9805097] [SPIRES].

[87] M. Bañados, C. Teitelboim and J. Zanelli, The black hole in three-dimensional space-time, Phys. Rev. Lett. 69 (1992) 1849 [hep-th/9204099] [SPIRES].

[88] J. Hansen and P. Kraus, Generating charge from diffeomorphisms, JHEP 12 (2006) 009 [hep-th/0606230] [SPIRES].

[89] B. Sahoo and A. Sen, $\alpha^{\prime}$-corrections to extremal dyonic black holes in heterotic string theory, JHEP 01 (2007) 010 [hep-th/0608182] [SPIRES].

[90] C. Vafa and E. Witten, A one loop test of string duality, Nucl. Phys. B 447 (1995) 261 [hep-th/9505053] [SPIRES].

[91] P.D. Prester, $A d S_{3}$ backgrounds from $10 D$ effective action of heterotic string theory, Phys. Rev. D 81 (2010) 044014 [arXiv:0912.0030] [SPIRES].

[92] P.D. Prester, $\alpha^{\prime}$-corrections and heterotic black holes, arXiv:1001.1452 [SPIRES].

[93] M. Cvitan, P.D. Prester and A. Ficnar, $\alpha^{\prime 2}$-corrections to extremal dyonic black holes in heterotic string theory, JHEP 05 (2008) 063 [arXiv:0710.3886] [SPIRES].

[94] P.D. Prester and T. Terzic, $\alpha^{\prime}$-exact entropies for BPS and non-BPS extremal dyonic black holes in heterotic string theory from ten-dimensional supersymmetry, JHEP 12 (2008) 088 [arXiv:0809.4954] [SPIRES].

[95] D. Kutasov, F. Larsen and R.G. Leigh, String theory in magnetic monopole backgrounds, Nucl. Phys. B 550 (1999) 183 [hep-th/9812027] [SPIRES].

[96] M. Cvetič and D. Youm, Dyonic BPS saturated black holes of heterotic string on a six torus, Phys. Rev. D 53 (1996) 584 [hep-th/9507090] [SPIRES].

[97] M. Cvetič and A.A. Tseytlin, Solitonic strings and BPS saturated dyonic black holes, Phys. Rev. D 53 (1996) 5619 [Erratum ibid. D 55 (1997) 3907] [hep-th/9512031] [SPIRES].

[98] D. Gaiotto, A. Strominger and X. Yin, New connections between $4 D$ and $5 D$ black holes, JHEP 02 (2006) 024 [hep-th/0503217] [SPIRES].

[99] A. Dabholkar, S. Murthy and D. Zagier, to appear.

[100] S. Banerjee and A. Sen, Duality orbits, dyon spectrum and gauge theory limit of heterotic string theory on $T^{6}$, JHEP 03 (2008) 022 [arXiv: 0712.0043] [SPIRES].

[101] S. Banerjee and A. Sen, S-duality action on discrete T-duality invariants, JHEP 04 (2008) 012 [arXiv: 0801.0149] [SPIRES].

[102] J.M. Maldacena, A. Strominger and E. Witten, Black hole entropy in M-theory, JHEP 12 (1997) 002 [hep-th/9711053] [SPIRES].

[103] J.A. Harvey, R. Minasian and G.W. Moore, Non-abelian tensor-multiplet anomalies, JHEP 09 (1998) 004 [hep-th/9808060] [SPIRES].

[104] P. Kraus and F. Larsen, Holographic gravitational anomalies, JHEP 01 (2006) 022 [hep-th/0508218] [SPIRES].

[105] P. Kraus, Lectures on black holes and the $A d S_{3} / C F T_{2}$ correspondence, Lect. Notes Phys. $\mathbf{7 5 5}$ (2008) 193 [hep-th/0609074] [SPIRES]. 